


\title{
Chiral phenomena in magnetic multilayers
}

\author{
E. van Walsem
}


PhD thesis, Utrecht University, March 2021

DOI: $10.33540 / 543$

About the cover: Artist impression of a 2d-spin system containing both structures who have a central role in this thesis: chiral spirals and skyrmions. Design and illustration by S. A. Brands. 


\title{
Chiral phenomena in magnetic multilayers
}

\author{
Chirale verschijnselen in meerlaagse magneten \\ (met een samenvatting in het Nederlands)
}

\section{Proefschrift}

ter verkrijging van de graad van doctor aan de Universiteit Utrecht op gezag van de rector magnificus, prof.dr. H.R.B.M. Kummeling, ingevolge het besluit van het college voor promoties in het openbaar te verdedigen op maandag 29 maart 2021 des middags te 12.45 uur

door

Etienne van Walsem

geboren op 31 juli 1991

te Maarssen 
Promotor: Prof dr. R. A. Duine

Dit proefschrift werd mogelijk gemaakt met financiële steun van de Stichting Nederlandse Wetenschappelijk Onderzoek Instituten (NWO-I, voorheen FOM), die deel uitmaakt van de Nederlandse Organisatie voor Wetenschappelijk Onderzoek (NWO). 


\section{Contents}

Chapter 1: Introduction 1

Chapter 2: Structural transitions of skyrmion lattices in synthetic antiferromagnets

Chapter 3: Layer effects on the magnetic textures in magnets with local inversion asymmetry

Chapter 4: Collective modes of skyrmions in synthetic antiferromagnets

Chapter 5: Outlook

Bibliography

$\begin{array}{ll}\text { A: Addendum } & 101\end{array}$

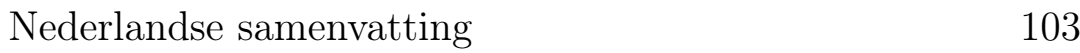

$\begin{array}{ll}\text { Dankwoord } & 109\end{array}$

$\begin{array}{ll}\text { About the author } & 111\end{array}$ 



\section{$1 \quad$ Introduction}

\subsection{Brief history of magnetism}

The understanding of magnetism has played a leading role in human behavior, from the compass used in the Chinese dynasties to the Giant Magnetoresistance being the fundamental ingredient of the hard disk drives in the current age of information. In this thesis we try to understand new phenomena in magnetic materials which hopefully lead to new applications for the future. In this section we will first lead you along the magnetic milestones and then go more in depth into ferromagnetism and atomic models.

Permanent magnets have been known to humankind in ancient Greece, China and the Americas in the form of lodestones $\left(\mathrm{Fe}_{3} \mathrm{O}_{4}\right)$. Around 20BC - 20AD, the first usage for these "magic" stones was found in China where they used a magnetic rod to point along the earth's magnetic field. The first artificial magnets were found by heating up Iron $(\mathrm{Fe})$ to above the Curie temperature and aligning to the earth magnetic field in 1064 by Zheng Gongliang [1]. Also, in what is regarded as the first scientific text treats magnets, W. Gilbert deduced that the compass aligns to the earth's magnetic field [2], and is not steered by the stars, as was thought before. In the 19th century big steps were made in the understanding of electromagnetism: Ørsted found out that a current-carrying electric wire yields a magnetic field, which was quickly adapted by Ampere and Arago who coiled such a wire and create an electro- 
magnet. This research pinnacled to the Maxwell equations in 1864 [3]. By now, the framework of electromagnetism is known. But, currently there is still a lot of active research in the field of permanent magnetism, especially considering magnetic multilayers. In this thesis we will study the effects of such magnetic multilayers.

To further explain permanent magnets we need to differentiate three types of magnetism. Diamagnetism implies a repulsive reaction to an applied magnetic field, Paramagnetism is associated with an attraction to an external magnetic field and lastly, ferromagnetism has a magnetic field independent of an applied field [4]. In this thesis we are investigating materials with ferromagnetic properties. An atomic theory of (ferro)magnetism should describe two distinct phenomena: hysteresis under an applied field and spontaneous magnetization below the Curie temperature. In Fig. 1.1 we show typical curves for both phenomena. The first fitting theory to describe permanent magnetism and agreeing with both phenomena was by Pierre Weiss in 1906 [5]. He made two assumptions: First, that the atom's magnetic dipole prefers to align with each other over (lower) thermal fluctuations, explaining the temperature dependence of magnets. Second, that group of atoms align in domains, and these domains are pointing in random directions meaning that the total magnetization would be zero. By applying an external magnetic field the domains would gradually align and this explains the hysteresis loop. These assumptions were made by Pierre Weiss without any foundation, but we now understand that this is a good physical picture of atomic behavior in magnetic systems.

The internal magnetic field in the Weiss model was multiple orders of magnitude larger than the total magnetization, which is greater than any field measured outside a magnet. To give this a satisfactory explanation we need to include quantum physics. The key ingredient which quantum physics includes in the atomic model of ferromagnetism is spin of the electrons. Each atom caries 
(a)

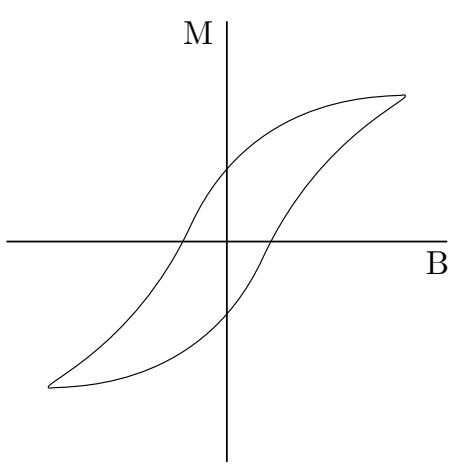

(b)

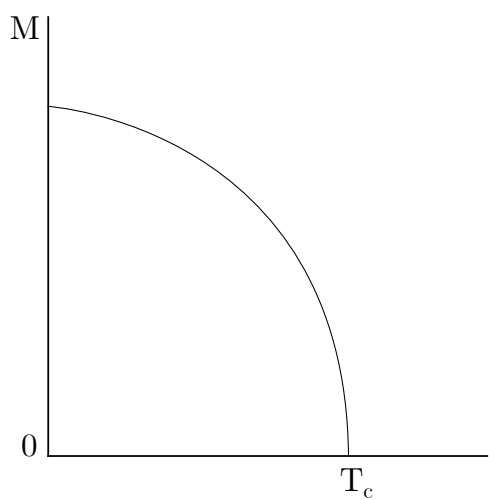

Figure 1.1: (a) magnetic hysteresis loop and (b) temperature dependence of an magnet showing the critical Curie temperature.

a magnetic moment originating from electrons orbiting around the nucleus and the quantum mechanical spin. It turns out that the contribution of the spin is far stronger than this orbital angular momentum. Therefore, permanent magnets are truly a quantum mechanical phenomenon. This claim is made even stronger by Niels Bohr and Hendrika van Leeuwen who proved that magnetism in classical physics is impossible altogether $[6,7]$.

Paramagnetism and ferromagnetism are comparable in that their atoms have non-zero magnetic moments, but the difference between the two is that the average moment of all spins is zero in the paramagnetic case and non-zero in the ferromagnetic case. To describe where this difference is coming from, we have to look at the interactions between the atoms. These interactions are the Heisenberg exchange interaction, where neighboring spins favour to align. The hamiltonian of Heisenberg exchange is written as:

$$
H=-J \sum_{\langle i, j\rangle} \vec{S}_{i} \cdot \vec{S}_{j}
$$


where $\vec{S}_{i}$ are the spins of the atoms, $J$ is the strength of the Heisenberg exchange interaction and $\langle i, j\rangle$ refers to a summation over neighboring spins only. We have a richness of models following from the Heisenberg model. $J>0$ is the ferromagnetic case. But for $J<0$ you get antiferromagnetic, or when considering two type of spins: ferrimagnetic systems (see Fig. 1.2).

As in the Weiss model, the spins in the $J>0$ Heisenberg model align, but in experimental settings domains form because of dipoledipole interactions. To have a macroscopic magnetization we have to break the rotation symmetry of the spins. We do this by adding an external magnetic field

$$
H=\vec{B} \cdot \sum_{i} \vec{S}_{i}
$$

where $\vec{B}$ is the strength of the magnetic field. Furthermore, certain materials have anisotropic behavior originating from the crystalline structure of the atoms or the shape of the material itself. Because of this anisotropy, the domains formed by dipole-dipole interactions have a preferred direction for their spins, for example either up or down. This symmetry is broken easier and thus these materials can be natural permanent magnets below the curie temperature.

Around 1930 the consensus was that most physics describing the quantum mechanical effects in condensed matter were considered known. Dirac even went far as saying: "The underlying physical phenomena necessary for a mathematical explanation of a large

(a)

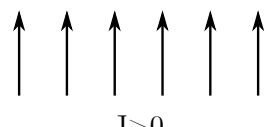

(b)

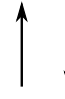

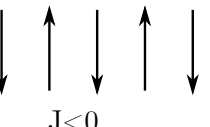

(c)

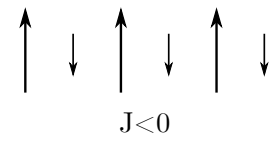

Figure 1.2: Ordering of spins in a Heisenberg model which is (a) ferromagnetic $(J>0)$, (b) antiferromagnetic $(J<0)$, (c) ferrimagnetic. 
part of physics and all of chemistry are now understood in principle, the only difficulty being that the exact application of these laws leads to equations much too complicated to be soluble" [8]. In this thesis we are still busy trying to solve the much too complicated equations and say something about state of the art magnetic compounds.

\subsection{Antiferromagnetism}

One of the applications of the Heisenberg model occurs as we consider the model for $J<0$. Here, all spins are oriented in an alternating order in the ground state, as drawn in Fig. 1.2 (b). This is referred to as Antiferromagnetic or Néel ordering, named after Louis Néel who first described this in 1936 [9] and was awarded a Nobel prize for this. Antiferromagnetism was postulated before experimental observation, this was found later in $\mathrm{Mn}^{++}$[10]. Since then it has been found in multiple materials, such as $\mathrm{Cr}, \mathrm{MnO}$ and $\mathrm{NiO}$ [10-12]. But Louis Néel was not very hopeful for use of the materials, he wrote in his Nobel price speech: "They (antiferromagnetic materials red.) are extremely interesting from the theoretical viewpoint but do not seem to have any applications." Nevertheless, multiple practical applications are found today. Lately, AFM materials have gotten a lot of attention in the spintronics community since they are relatively robust against external magnetic fields [13]. Furthermore, one of the most well known applications is the Giant Magneto Resistance (GMR), which we will treat later. GMR is dependent on Synthetic Antiferromagnets.

\subsubsection{Synthetic Antiferromagnets}

Synthetic antiferromagnets (SAFs) consists of ferromagnetic layers with a spacer of normal metal in between. For clarification we show a cartoon of this in Fig. 1.3. The ferromagnetic layers are coupled by spin dependent Ruther-Kittel-Kasuya-Yosida-coupling 


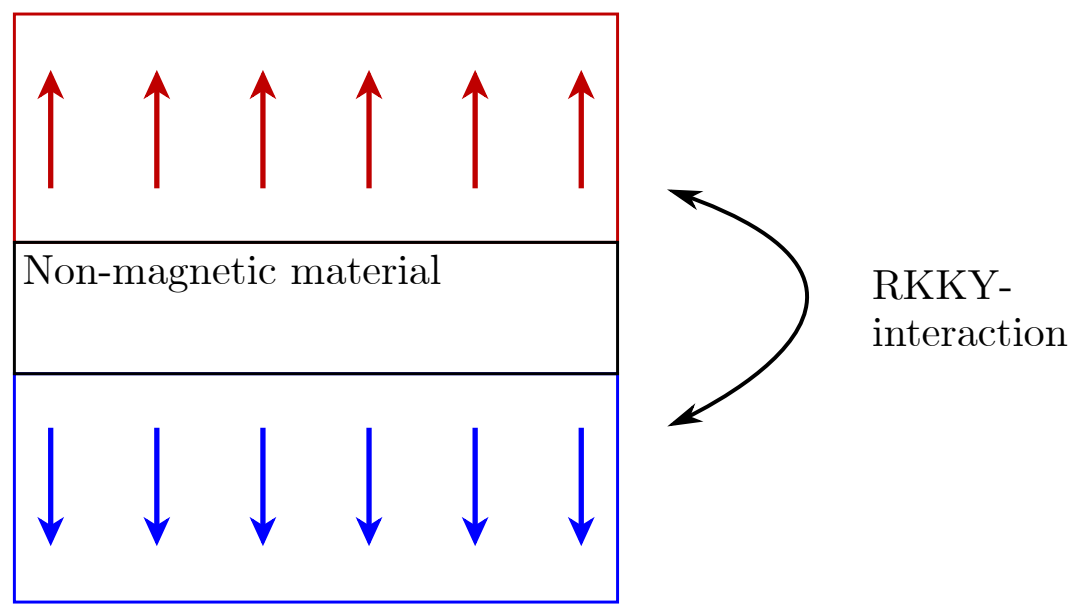

Figure 1.3: Schematic drawing of a Synthetic Antiferromagnet $(\mathrm{SAF})$.

(RKKY-coupling). This can be either positive or negative, dependent on the size of the spacer [14-18]. The advantage of SAFs is that we can engineer them such that their properties are suitable for applications. Furthermore, we can influence the SAFs, e.g. by applying pressure [19, 20] or an electric field [21], such that their properties are tunable: we can increase/decrease the interlayer coupling or even switch the sign of the coupling. In this thesis we show multiple examples of how SAFs can be used to influence magnetic textures.

\subsection{Information carriers}

While society is getting more dependent on information technology, new techniques need to be developed to keep up with the demand. Because, following the famous Moore's law, also the supply and demand of computer memory increased exponentially since the 70's, as shown in Fig. 1.4, and we do not expect that the cur- 
rent techniques are capable to sustain this growth. Historically speaking, important computer memory techniques are based on magnetic materials. One of the most prolific methods is magnetic tape. This method, best known for recording music, is still used to this day for backup of digital data of large servers. Magnetic tape consists of a thin sheet of plastic with on one side magnetic iron oxide. The information is written on the tape by the magnetization direction of the iron oxide, e.g. for digital data you can interpret magnetic domains pointing "up" as ones and pointing "down" as zeros. This can be read out by leading the tape along a read out head containing a coil which induces an electrical current from the magnetic flux of the passing tape. Writing data is possible by reversing the order: put an electrical current through the coil and the induced magnetic field will change the tape's magnetic structure.

Another magnetic computer memory technique is the bubble technology. In a two dimensional slab of magnetic garnet, the combination of dipole-dipole interactions and boundary effects lead to the formation of domains in circular shapes, i.e. bubbles. These bubbles have a diameter of around $1 \mu \mathrm{m}$ [23] and have a different magnetization direction compared to the rest of the otherwise polarized slab. In Fig. 1.5 we show a cartoon of such a bubble. The bubbles can be moved trough the system by an electrical current. In this way information could be stored and moved around trough the system. Big advantages of bubble memory are: non volatility, meaning that the memory remains intact when the power of the memory is off, and the absence of moving parts, since only a magnetic field is needed to operate the memory. In the end, the bubble memory has never been widely adopted because of the low performances compared to its competitors: hard disk drives and flash memory turned out to be much faster, cheaper and have higher information density.

Today, modern computer storage consists of hard disc drives and solid state drives. Here we will focus on the hard disc drives. 


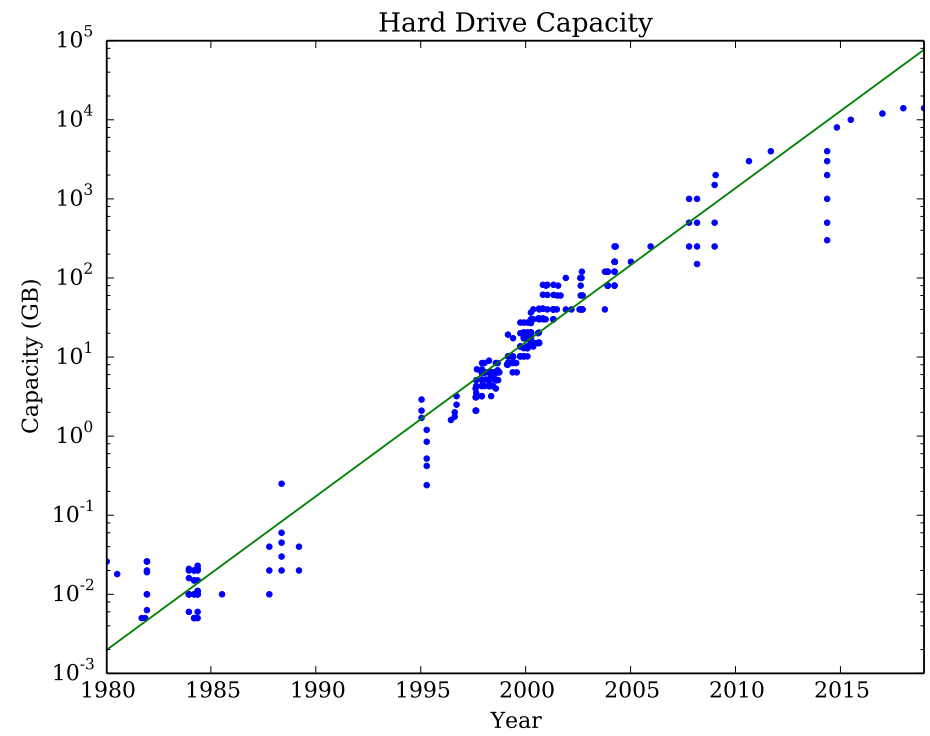

Figure 1.4: Example of a memory analogy of Moore's law, the capacity of hard disk drives (HDD) been rising exponentially over time. Data from [22]. 


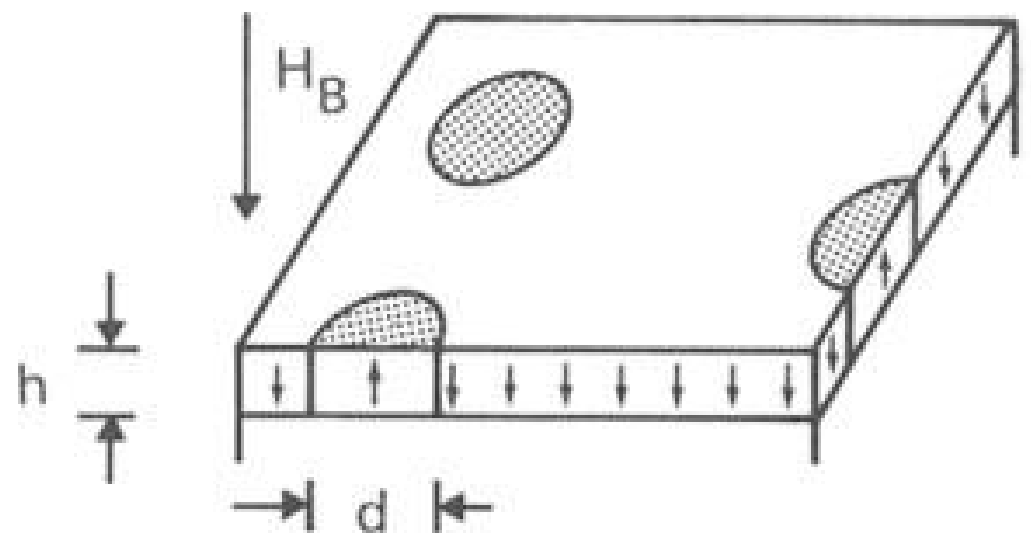

Figure 1.5: Schematic drawing of isolated magnetic bubbles. $H_{\mathrm{B}}$ is an external magnetic field, $h$ the thickness of the two dimensional garnet slab and $d$ the diameter of the bubble. Taken from [24].

They consist of a disc divided in small magnetic domains pointing in opposite directions, this is all read out or written on by a magnetic head comparable to those of the magnetic tape. The head is mounted on an arm which can move above the disc to reach different parts of the disc just like the mechanics of an old record player. To improve the sensitivity of the read out head, giant magnetoresistance (GMR) is used. This resistance has a very strong dependence on the magnetic alignment in the head, and by using spin valves this alignment is easily influenced by an external magnetic field [25]. Therefore, the read our head is much more sensitive to small changes of the magnetization in the hard disk. The discovery of GMR is attributed to Albert Fert and Peter Grünberg [26, 27]. In GMR multiple magnetic layers are stacked on top of each other to form a synthetic antiferromagnet. The resistance of such a system is dependent on the relative magnetization of the layers: parallel magnetization of the layers gives a higher resistance then antiparallel magnetized layers. The difference of the resistance is up 


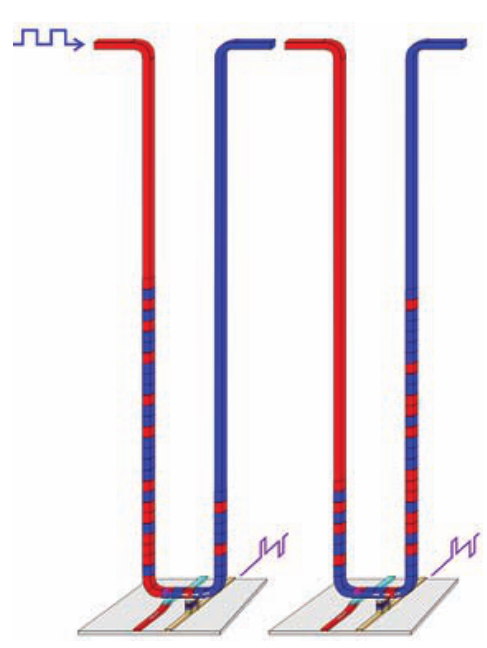

Figure 1.6: Drawing of the design of a racetrack memory. From [30].

to $220 \%$ [28], this is considered giant. The principle behind GMR originates from the scattering of electrons. There are two ways the electron scatter: First, there is scattering at the surfaces between the opposite polarized layers. Due to the different polarizations of the layers there is a strong exhange field around the surface which is responsible for the scattering. Second, inside the ferromagnetic layers the electrons scatter from the magnetic atoms, dependent on the spin of the electron and the atom. By having multiple orientations of ferromagnetic layers the probability to scatter increases. From a physicist standpoint it is charming to note that GMR is one of the first applications where spin and charge of electrons is combined instead of treated separately and can be considered as the launch of a new field of research: spintronics [29].

One of the new proposals for computer memory is called the magnetic racetrack memory [30]. This technique has a higher storage density than solid state drives and has a faster read write 
speed than hard drives [31]. Magnetic racetracks brings elements of the techniques mentioned above together: A long solid strip of magnetic material can decode computer bytes. The bits are stored through magnetisation direction just as in the magnetic tape. Only here, the magnetization pattern is moved through the system by an electrical current, analogues to how bubbles are moved by an magnetic field in bubble memory. In a racetrack, the domains can reach speeds above $1500 \mathrm{~m} / \mathrm{s}$ [32]. A schematic drawing is shown in Fig. 1.6. For the pattern to be moved trough the system without loss of shape and thus information, they need to be very stable. This is achieved by topological protection, see Section 1.4, either by a domain wall or by using skyrmions [33], which is a smaller, more stable bubble. Elemental to creating the topological protection of the patterns is a layering of magnetic layers just as in a SAF. Finally, there is an added benefit of the magnetic race track memory: the magnetic strips can be folded in a U-like structure. In this way the racetrack uses three spatial dimensions which is beneficial over other techniques that only use two.

\subsection{Topological structures}

All research in this thesis can be traced back to the effects of the Dzyaloshinskii-Moriya interaction, which produces rich and surprising effects in magnetic materials. Dzyaloshinskii-Moriya interaction was first described as an explanation why $\alpha-\mathrm{Fe}_{2} \mathrm{O}_{3}$ was showing weak ferromagnetic behavior despite having an antiferromagnetic ordering of its spins. Dzyaloshinskii showed phenomenologically in [34] that spins placed perpendicular to the trigonal axis with an AFM order have the same symmetry as canted spins which have a net magnetization perpendicular to the trigonal axis. Writing out the hamiltonian for this case, we see that it includes the term

$$
\vec{D} \cdot\left[\vec{S}_{i} \times \vec{S}_{j}\right]
$$




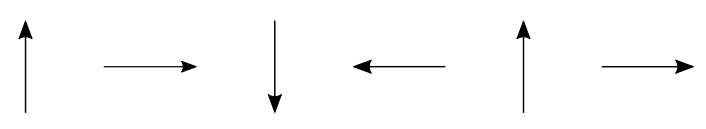

Figure 1.7: 1D spin structure only factoring DMI $\vec{D}=(1,0,0)$

which is essential to form the canted spin arrangement, and thus the weak ferromagnet. Also it proves that the canted spin configuration is the more favorable configuration. Shortly afterwards, Moriya showed that this effect was coming from the spin-orbit coupling [35]. He described this as the anti-symmetric part of the anisotropic super exchange interaction, and from his calculations it is possible to calculate the strength of $\vec{D}$ which was not possible with Dzyaloshinskii's. Later, an interaction of the form as in Eq. (1.3), was called the Dzyaloshinskii-Moriya interaction, or in short: DMI.

In 1980 A. Fert showed that DMI is important in doped CuMn since there is an addition of RKKY interaction of the DMI type in the interaction between conduction electrons and non magnetic (heavy metal) particles [36]. Essential in these materials is a Structural Inversion Asymmetry, often referred to as a broken inversion symmetry, and a strong spin-orbit coupling. In this thesis we study materials having these same two requirements.

So how does the DMI influence a simple, understandable system? For example, let us take a one-dimensional spin chain. With classical spins $\vec{S}$ of unit length who rotate in three dimensions. If the only interaction between the spins in there is the $\vec{D}=(1,0,0)$ DMI, every next spin in the chain wants to orient perpendicular to the previous spin to minimize the DMI. The direction is always the same and we end up with a spiral configuration as shown in figure Fig. 1.7. This spiraling behavior and chirality are the important takeaways for our thesis which originate from the DMI. 


\subsubsection{Domain walls}

If we extend our spin chain example from our previous section by including nearest neighbor exchange interaction and anisotropy we will get a configuration including polarized domains and so called domain walls in between them. The nearest neighbor exchange interaction between two spins, is described in Eq. (1.1) and favors the spins to be aligned. The anisotropy is written as

$$
K\left(S_{z}\right)^{2},
$$

where $K$ is the strength of the anisotropy and $\vec{S}_{z}$ is the z-direction of the spin. This anisotropy favors one axes for the polarized spins to point along, in this case this is the z-axis and is perpendicular to the spin chain. Therefore this is referred to as the Perpendicular Magnetic Anisotropy, or PMA. For a longer spin chain, domains of spins oriented along both directions of the anisotropy axis will form, and the border between these domains are thus called domain walls.

The influence of the DMI is that each domain wall always turns in the same direction. See Fig. 1.8 for a schematic drawing of such domain walls. This chirality protects the domains since the walls can not be continuously deformed such that the wall disappears. In contrast with two opposite rotating walls, where they can be fused together to annihilate each other. The mathematical study of objects that can not be continuously deformed is called topology, and in physics we say that these domain wall structures are topological protected. This can be described more rigorously. If we start with two domain walls in a spin chain with spins pointing up at both ends, we can map the direction of every spin to an $S^{1}$ configuration, where $S^{1}$ denotes the unit circle. Mathematically speaking, this mapping is classified by the homotopy group $\pi_{1}\left(S^{1}\right)=\mathbb{Z}$. This means that we can compute the winding number $w$ of the domain 
wall by

$$
w=\frac{1}{2 \pi} \int \vec{m}^{\perp} \cdot \frac{\partial \vec{m}}{\partial x} d x,
$$

where $\vec{m}$ is the continuous magnetization of the spins, $\vec{m}^{\perp}$ the vector perpendicular to the magnetization ${ }^{1}$ and we integrate in the direction of the line, $x$. This winding number is exactly 1 for a pair of protected domain walls, and 0 if they are not topologically protected. It is important to notice two things. First, we treat the magnetization of the spins as two-dimensional, which is valid for sufficiently large $\vec{D}$ because the vector $\vec{D}$ forces the spins to rotate in one plane only. And second, the integral evaluates to 1 only for a full rotation, since a well defined system has spins pointing in the same direction at infinity. Therefore, a single domain wall as depicted in Fig. 1.8 is only half a rotation and has thus a winding number of $w=1 / 2$.

There are two kinds of domain walls originating from the DMI. As one can see from Eq. (1.3) the cross product prefers two neighboring spins to orient perpendicular with respect to each other. Seen from the first spin $\vec{S}_{i}$ there is a whole plane oriented perpendicular. The vector $\vec{D}$ determines how the second spin $\vec{S}_{j}$ is oriented in this perpendicular plane. The plane has two directions: parallel and perpendicular to the spin chain. For the first parallel option the vector $\vec{D}=(1,0,0)$, which leads to a so called Néel domain wall, and for the second perpendicular option $\vec{D}=(0,1,0)$, we end up with a Bloch domain wall.

\subsubsection{Skyrmions}

For this section we want to extend our spin chain to a full twodimensional magnetic slab with DMI, nearest neighbor exchange, anisotropy and an external magnetic field. In the case that the

\footnotetext{
${ }^{1}$ There are of course two perpendicular vectors possible, but here we choose just one.
} 

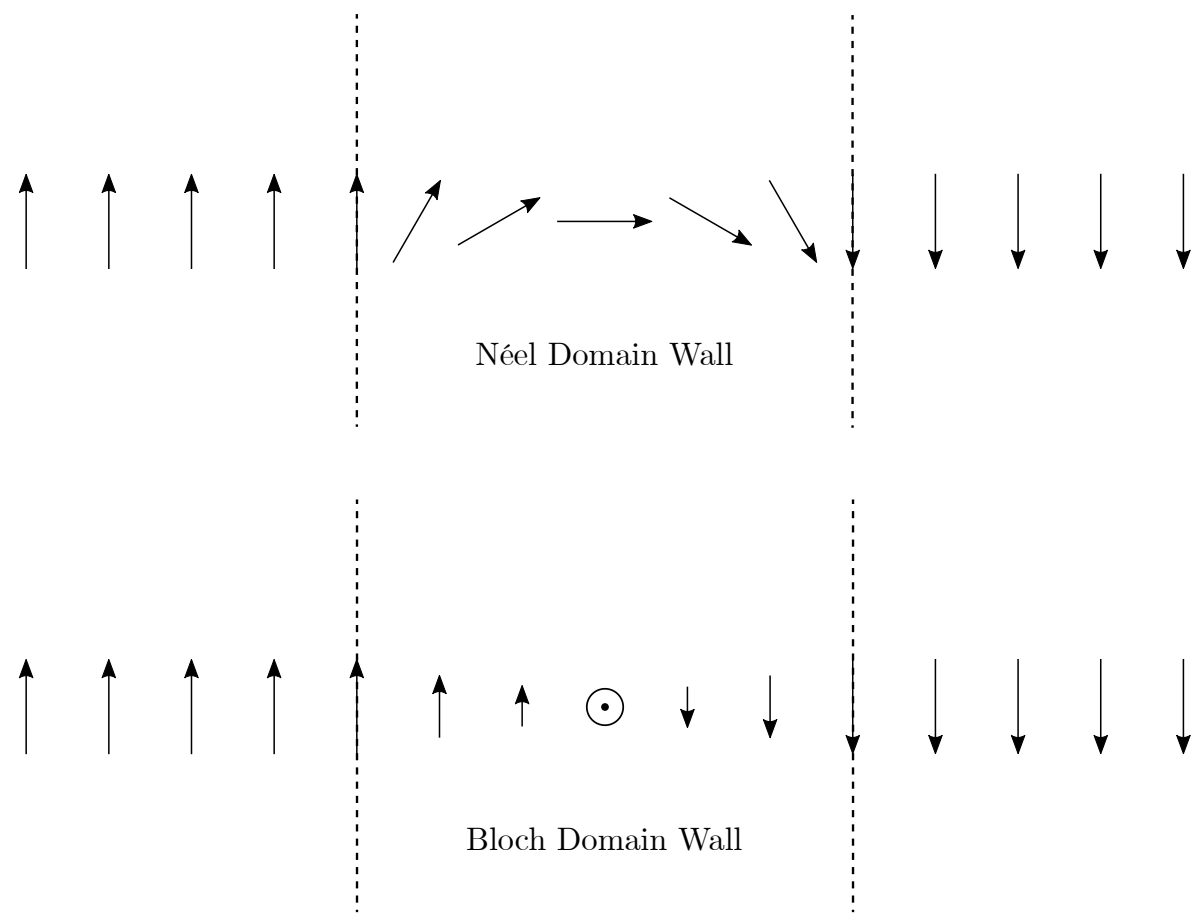

Figure 1.8: Schematic drawing of (a) Néel domain wall and (b) Bloch domain wall 


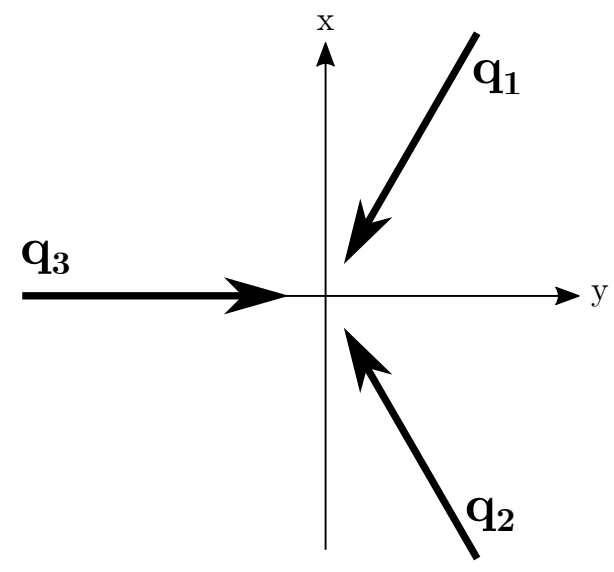

Figure 1.9: Schematic drawing of three wave vectors of spirals, oriented towards each other with equally spaced angles between them. These three spirals together form a triangular vortex lattice.

DMI is large enough magnetic spirals will form and can point, in principle, in all directions along the slab. If we have three of those spirals with directions $\mathrm{q}_{1}, \mathrm{q}_{\mathbf{2}}$ and $\mathrm{q}_{\mathbf{3}}$, we can form a lattice of vortex-like structures by orienting these spirals towards each other with equally divided angles between the spirals, as drawn in Fig. 1.9. This vortex lattice is analogous to the Abrikosov lattice in superconductors. It can be shown that for the right parameters this lattice has a more favorable energy than a stripe pattern [37]. This vortex structure is solitonic in nature and is called a skyrmion. In Fig. A.2 we show such a skyrmion structure. Also here, both options of DMI vectors can give you either Néel or Bloch skyrmions. Néel skyrmions are formed in thin magnetic films, while Bloch skyrmions are formed in bulk magnets where the DMI has a different vector $\vec{D}$.

We can map all the spins of a skyrmion to an $S^{2}$ configuration, just like we could map the spins of a domain wall to an $S^{1}$ structure. 
(a)

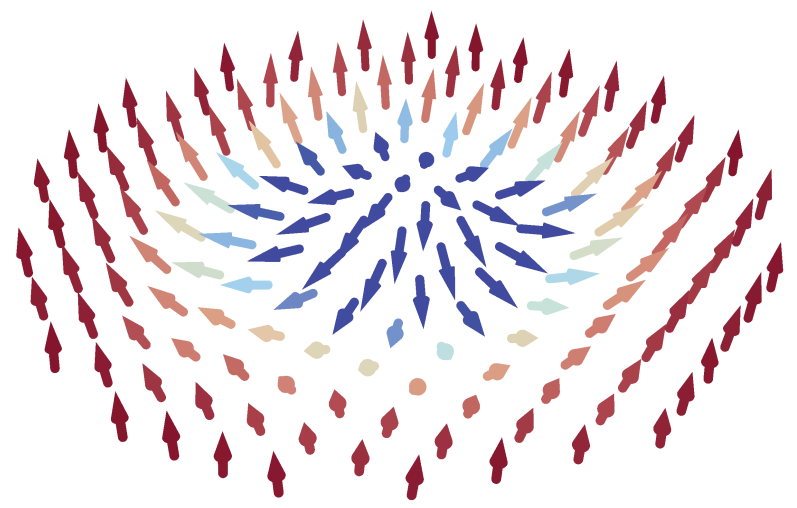

(b)
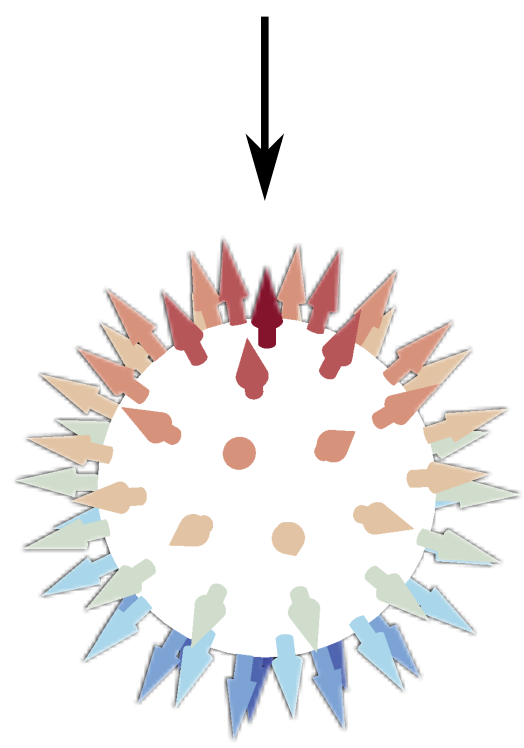

Figure 1.10: Schematic drawing of a skyrmion and the map of a skyrmion to a sphere $S^{2}$. 
We can also calculate a winding number in two dimensions by

$$
w=\frac{1}{4 \pi} \int \vec{m}\left(\frac{\partial \vec{m}}{\partial x} \times \frac{\partial \vec{m}}{\partial y}\right) d x d y,
$$

which gives a value of exactly 1 for skyrmions. You can view the skyrmion as a two-dimensional extension of the domain wall, but classified by homotopy group $\pi_{2}\left(S^{2}\right)=\mathbb{Z}$. The name Skyrmion is derived from high energy physics where Tony Skyrme used solitonic solutions in the non-linear $\sigma$-model to describe a meson field where topological protected structures emerged as baryons [38]. There is a difference: the high energy skyrmions have homotopy group $\pi_{3}\left(S^{3}\right)=\mathbb{Z}$ and our magnetic skyrmions have the homotopy group $\pi_{2}\left(S^{2}\right)=\mathbb{Z}[39]$. Therefore, some people prefer to refer to magnetic skyrmions as baby skyrmions instead of just skyrmions. The topological protection of skyrmions makes them interesting for the information technologies mentioned in Section 1.3.

The condensed matter skyrmion renaissance started in 2009 with the first detection of Bloch skyrmions in MnSi [40], thereafter a flurry of new types of materials containing skyrmions was found such as thin film ferromagnets $\left(\mathrm{Fe}_{0.5} \mathrm{Co}_{0.5} \mathrm{Si}\right.$, Fe on $\left.\operatorname{Ir}(111)\right)$ [41, 42], doped semiconductors $\left(\mathrm{Fe}_{1-\mathrm{x}} \mathrm{Co}_{\mathrm{x}} \mathrm{Si}\right.$ for $\left.x=0.2\right)$ [43] and also in helimagnets at higher temperature such as FeGe [44]. Now they are even found at room temperature which shows their suitability for technological applications [45]. Furthermore we like to note here that DMI is not the only way to form skyrmions, it has been shown that competing Heisenberg exchange interactions in frustrated magnets [46], magnets with double-exchange interactions [47] and quantum hall systems [48, 49] also possess the necessary properties to form skyrmions.

\subsubsection{Hopfions}

As we have seen, we can have topological structures in one dimension and two dimensions, the logical follow-up question would be 
what a three-dimensional topological soliton structure would look like. We can create such a structure in a three-dimensional ferromagnet with nearest neighbor exchange interactions, DMI and shape anisotropy. A three-dimensional structure itself is a bit difficult to draw but it is composed as follows: we start with a string of skyrmions. Now we take one end of this string and twist it a full revolution, and thereafter join the two ends of the twisted skyrmion string [50]. This donut-like shape is called a hopfion and is solitonic by nature. In Fig. 1.11 we plotted the magnetization of two different intersections of a hopfion, where in (a) you can see that the hopfion is indeed a donut-like shape, and in (b) that the string in both sides of the donut consists of a skyrmion. On the boundary of the hopfion the magnetization has to have the same value, in this case all pointing in the positive z-direction. The topology of a hopfion is equivalent to a closed ball $S^{3}$, and the magnetization is a map classified by the homotopy group $\pi_{3}\left(S^{2}\right)=\mathbb{Z}$. The topological charge of a hopfion, the Hopf charge, is calculated different than the previous winding numbers. Here, a magnetic field line is the line composed of spins pointing in the same direction, i.e. the line of constant magnetization. In Fig. 1.12 we plotted three of such lines. The Hopf charge is defined by the number of links between any of two lines in a structure. Thus, in this case the Hopf charge is 1 . Hopfions can be a promising candidate for information technologies. 
(a)

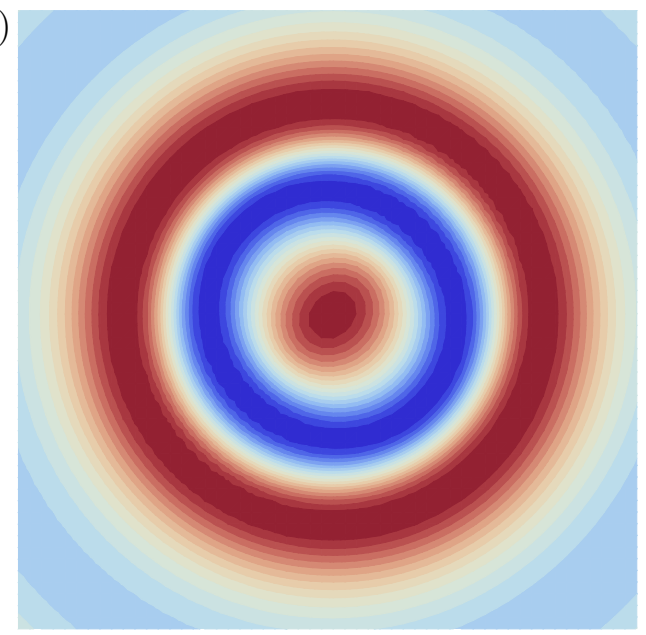

(b)

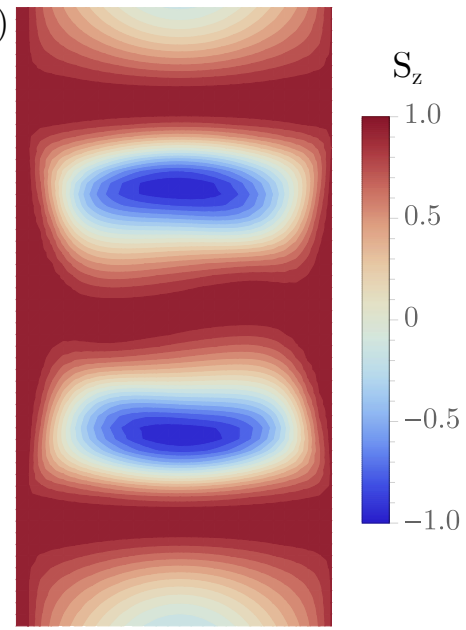

Figure 1.11: Cut trough of a hopfion in the (a) x-direction and (b) z-direction.

\subsection{Monte Carlo simulations}

Most results in this thesis are obtained using Monte Carlo simulations. But what is special about Monte Carlo simulations compared to other numerical methods? The error of most numerical methods scales with the number of dimensions, $d$, as $N^{-1 / d}$, where $N$ is the number of points of your simulation. As an example, the number of possible states of a simple Ising spin model goes as $2^{n}$, with $n$ the number of spins. And, in this thesis we treat systems with up to 327.680 spins. Thus, with the speed of our current computers we would need a lot more patience to simulate such a system than a 4 year Ph.D. project can provide. Luckily, there are Monte Carlo simulations where the error scales independent of the dimensionality as $1 / \sqrt{N}$ and is a good method to probe our systems with extreme high dimensions [51].

Monte Carlo is a probabilistic simulation method. Einstein said that God does not play dice, but in Monte Carlo simulations we 


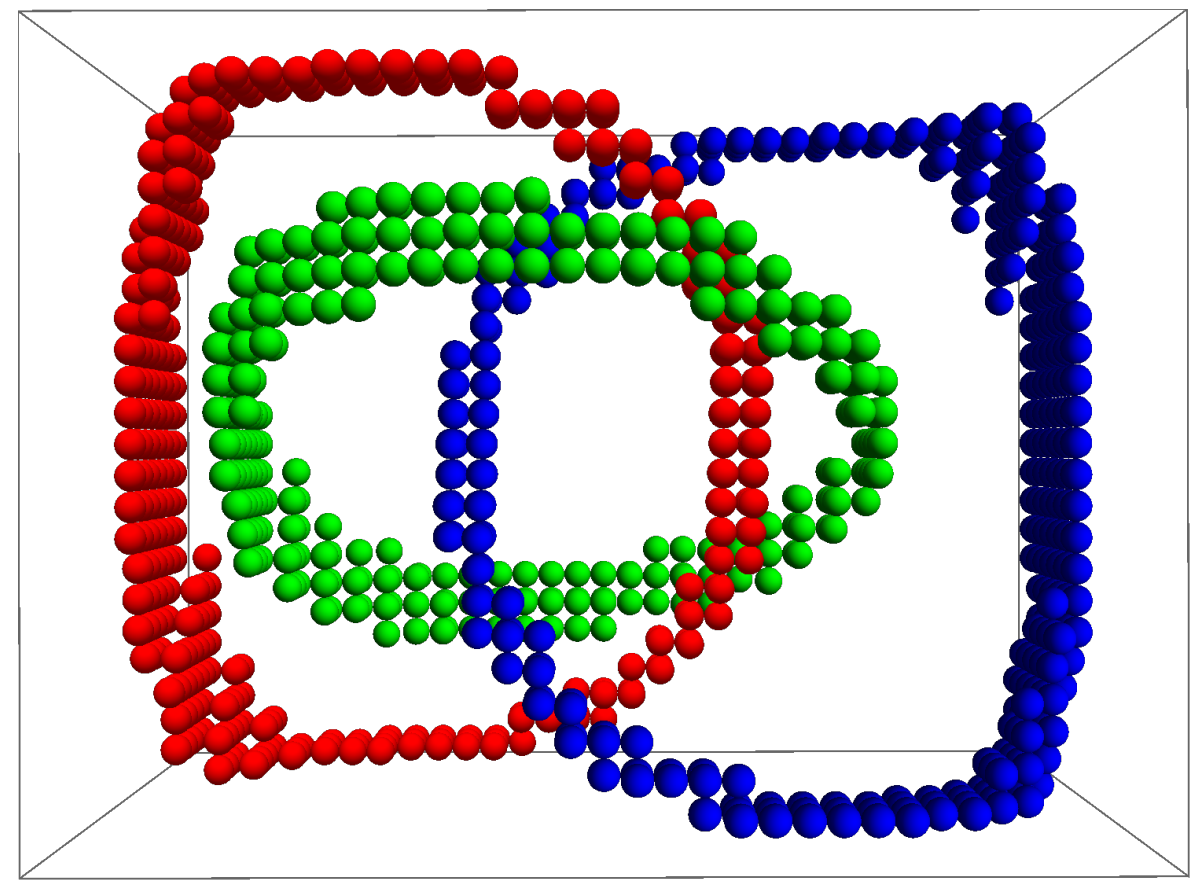

Figure 1.12: Schematic drawing of a skyrmion and the map of a skyrmion to a sphere $S^{2}$. 
(a)

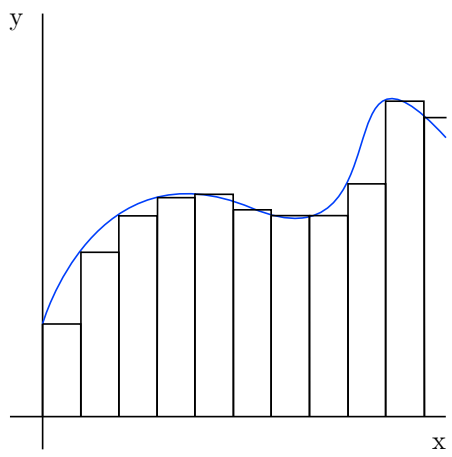

(b)

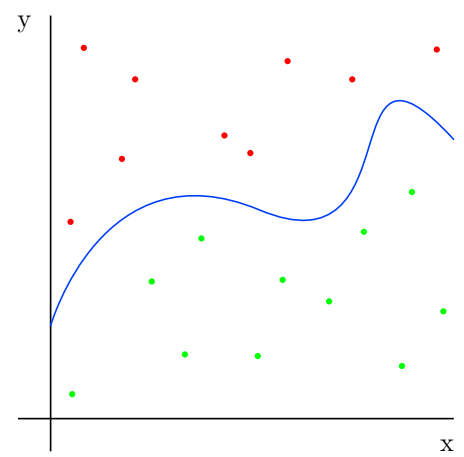

Figure 1.13: Schematic drawing of (a) a Riemann integral where the area under the continuous curve is approximated by a finite sum of $\mathrm{N}$ rectangles, and (b) a Monte Carlo simulation calculating the same area under the curve, where we approximate this by the ratio between the total number of points, $N$, and the number of points under the curve (green).

use this dice to say something about physics. Where people in the Monte Carlo casino bet on the random outcomes of the die, we use these random numbers to estimate integrals and sums. For example, we can estimate the area under a curve by mapping the random outcome of the die on coordinate system, and then count the number of random coordinates placed under or above the curve, as drawn in Fig. 1.13. But, to determine the partition function of a spin system we need more sophisticated methods, for our system we use methods comparable to the Metropolis algorithm.

Let us explore all of this more rigorously and focus more on spin systems to explain this Metropolis algorithm. We want to calculate observables from the partition function,

$$
Z=\sum_{\left\{s_{i}\right\}} e^{-\beta E_{i}}
$$

where $\left\{s_{i}\right\}$ are all possible spin configurations, $\beta$ the inverse tem- 
perature $1 /\left(k_{B} T\right)$ dependent on the Boltzmann constant $k_{B}$ and temperature $T$, and $E_{i}$ the energy corresponding to spin configuration $s_{i}$. Now $w_{i}=e^{-\beta E_{i}}$ can be viewed as the weight of a spin configuration $s_{i}$ and the probability $P_{s_{i}}$ to find this weight is

$$
P_{s_{i}}=\frac{1}{Z} e^{-\beta E_{i}} .
$$

Subsequently, we use this probability to do importance sampling to speed up the process. Furthermore, for correct sampling of our physical system we need to obey two conditions. The first condition is ergodicity, where we need to be able to reach all possible states in our simulation with a realistic probability. And second, detailed balance needs to be fulfilled by,

$$
\frac{P_{i \rightarrow j}}{P_{j \rightarrow i}}=\frac{P_{j}}{P_{i}} .
$$

For Metropolis Monte Carlo simulations [52, 53] it is important that we can view the simulation as a Markov process of all possible spin configurations. We can do so since the probability to go from one to another state is

$$
P_{i \rightarrow j}=P_{s_{i}} / P_{s_{j}}=e^{-\beta\left(E_{i}-E_{j}\right)} .
$$

Thus this probability is only dependent on the states $s_{i}$ and $s_{j}$, and is independent of all other history, which are the requirements for a Markov process. On this the Metropolis algorithm is built where we propose a new spin configuration $s_{j}$ and accept this with the transition probability

$$
P_{i \rightarrow j}= \begin{cases}e^{\beta E_{i j}}, & E_{i j}<0 \\ 1, & \text { else }\end{cases}
$$

where $E_{i j}=E_{i}-E_{j}$. In essence, the Metropolis algorithm does importance sampling using this probability. Furthermore, this algorithm allows us to examine parts of a large phase space that are 
relevant at a chosen temperature. Also, if the starting point of the simulation is out of equilibrium the systems will thermalize after enough time.

\subsection{This thesis}

This thesis consists of three main chapters: In Chapter 2 we consider a two-layer SAF containing skyrmions: two ferromagnetic layers with DMI, anisotropy and an external magnetic field, coupled by interlayer exchange. Using Monte Carlo simulations we found the phase diagram for where the skyrmions form and we found that for a sufficiently large antiferromagnetic coupling the crystal structure of the skyrmions can change from triangular to square.

Chapter 3 focuses on SAFs with a varying number of layers. We describe a system where only DMI, interlayer and intralayer exchange interactions are present. The DMI in each subsequent layer has an opposite sign leading to an alternating pattern. Because of the presence of this DMI, a spiral magnetic structure forms. We found three different phases: the first where each layer has a spiral with a turning sense corresponding to the DMI of the concerning layer, the second where all spirals have a turning sense corresponding to the average DMI of the whole system and lastly, no spirals at all.

And finally, in Chapter 4, we return to skyrmions. Here, our system consists of two ferromagnetic layers, each containing one skyrmion exactly, coupled by interlayer exchange coupling. We discuss the dynamic properties of the two skyrmions by using the principle of least action analytically, and solve the Landau-Gilbert equation numerically for a ground state obtained by Monte Carlo simulations. We present the eigenfrequencies of the skyrmions and discuss them and the influence of the interlayer exchange coupling on them using both methods mentioned. 


\section{Structural transitions of skyrmion lattices in syn- thetic antiferromagnets ${ }^{1}$}

\subsection{Introduction}

Skyrmions in magnetic materials have been attracting great interest recently. Skyrmions were first introduced in particle physics and correspond to a classical stationary solution of the equations of motion with which a topological invariant is associated [38]. Later, such topological configurations were considered in magnetic systems by Bogdanov and Hubert [37]. Skyrmions can be small, in the nanometer range, and behave as (pseudo)particles that can be moved without decaying. Mühlbauer et al. demonstrated their existence with neutron scattering in $\mathrm{MnSi}$ in 2009 [55]. Later, thin magnetic multilayers proved to be able to possess magnetic skyrmions [41, 42, 56]. Initially, skyrmions were found at low temperatures but recently also room-temperature skyrmions have been created experimentally [45, 57-60]. Due to their solitonic behaviour and their rigidity originating from their topological prop-

\footnotetext{
${ }^{1}$ The contents of this chapter are based on the work of E. van Walsem, R.A. Duine, J. Lucassen, R. Lavrijsen and H.J.M. Swagten [54]. EvW, JL, RL, HS and RD conceived the project. EvW carried out all computations and wrote the initial manuscript. All authors commented on the manuscrip.
} 
erties they hold great promise for information technologies [30, 33, 61]. For example, skyrmion race track memory systems are being developed in which skyrmions act as bits and in which their postion is manipulated by current [33, 62, 63].

One of the classes of materials for hosting skyrmions are thin magnetic multilayers, such as $\mathrm{Co} / \mathrm{Pt}$, with high perpendicular magnetic anisotropy (PMA). The PMA is stronger than the inplane anisotropy thus providing opportunities for skyrmions since the spin in the centre of a skyrmion is oriented perpendicular to the layer as well. Furthermore, Dzyaloshinskii-Moriya interactions (DM interaction or DMI) are essential for forming skyrmions in thin magnetic films $[34,35]$. DMI needs two conditions to form: breaking of inversion symmetry and spin-orbit coupling. The stacking of different materials in the multilayers satisfies the first condition. To comply with the second condition, heavy metals such as $\mathrm{Pt}$ or Ta are used in the multilayers [64]. DMI can have different forms. The interfacial DMI that arises in magnetic multilayers stabilises Néel skyrmions, whereas the bulk DMI, in e.g. MnSi, stabilises Bloch skyrmions.

Examples of multilayer systems are synthetic antiferromagnets (SAFs) [65]. SAFs consist of two ferromagnetic multilayers which are coupled antiferromagnetically through the spin-dependent RKKY coupling [66-68]. By changing the thickness of the spacer, the coupling between the layers is oscillatory from antiferromagnetic to ferromagnetic and the magnitude is also dependent on the thickness of the spacer [14-18, 69]. Additionally, it has been shown that the coupling can be tuned with an external electric field [21]. The interlayer exchange coupling is much weaker than the exchange coupling within the layers so the antiferromagnetic order can compete with external fields and anisotropy. Zhang et al. have shown that SAFs are promising for developing the skyrmion race track memory because the Magnus force, which influences the direction of a moving skyrmion, is opposite in the different 
layers and thus cancels out [70]. Furthermore, exchange coupling between layers has been shown to stabilise skyrmions [60, 71].

In this paper we consider skyrmion lattices in synthetic antiferromagnets. We find that skyrmion lattices still occur for both synthetic ferromagnetic and synthetic antiferromagnetic coupling, although in the synthetic antiferromagnetic case a bigger magnetic field is needed to stabilise skyrmions. In addition, we find a structural phase transition in the synthetic-antiferromagnetic case from a triangular lattice to a square lattice. Skyrmion lattices in multilayer systems usually have a triangular configuration [37, 41, 55] although examples of square lattices also exist [42]. Similar transitions between triangular and square skyrmion lattices under magnetic field have been reported before in $\mathrm{MnSi}$ [72], Centrosymmetric Magnets [73] and 2D layers [74]. In $\beta$-Mn-type $\mathrm{Co}_{8} \mathrm{Zn}_{8} \mathrm{Mn}_{4}$ the same transition is found for cooling in an applied magnetic field [75]. Analogous to skyrmions, similar transitions between triangular and square lattices are observed in vortex lattices in two component Bose Einstein Condensates, where the two components play the role of the two layers [76]. In contrast to the examples above, the structural phase transition in SAFs has a possibility to be tuned in situ by altering the coupling between the layer by an electric field. Furthermore, in systems where also the DMI varies between the layers more exotic lattice configurations are achievable. Our research thus forms a route to tailoring skyrmion-lattices configurations.

In Section 3.2 we discuss our model and the simulations. After this we show results of our simulations in Section 3.3 and in Section 2.4 we discuss these results. Finally, we conclude with an outlook in Section 3.4. 


\subsection{Model and method}

We model the two magnetic layers by classical Heisenberg spins with ferromagnetic nearest neighbour coupling $J_{\mathrm{xy}}$, easy-axis anisotropy $K$ along $z$ and the Dzyaloshinskii-Moriya interaction $D$. Furthermore, both layers experience a uniform magnetic field $B$ and are coupled to each other with interlayer exchange $J_{z}$. In each layer the spins are positioned on the basis vectors of the simple square lattice $\hat{\mathbf{x}}, \hat{\mathbf{y}}$. We apply periodic boundary conditions in the $x$ and $y$-direction and open boundary conditions in the $z$-direction. The Hamiltonian is given by the sum of an interlayer and an intralayer part:

$$
H=H_{\text {intra }}+H_{\text {inter }} .
$$

The intralayer part is expressed as:

$$
\begin{aligned}
H_{\text {intra }}= & -J_{\mathrm{xy}} \sum_{\alpha \in\{T, B\}} \sum_{\mathbf{r}} \mathbf{S}_{\mathbf{r}}^{\alpha} \cdot\left(\mathbf{S}_{\mathbf{r}+\hat{\mathbf{x}}}^{\alpha}+\mathbf{S}_{\mathbf{r}+\hat{\mathbf{y}}}^{\alpha}\right) \\
& +K \sum_{\alpha \in\{T, B\}} \sum_{\mathbf{r}}\left(\mathbf{S}_{\mathbf{r}}^{\alpha} \cdot \hat{\mathbf{z}}\right)^{2}-\mathbf{B} \cdot \sum_{\alpha \in\{T, B\}} \sum_{\mathbf{r}} \mathbf{S}_{\mathbf{r}}^{\alpha} \\
& -D \sum_{\alpha \in\{T, B\}} \sum_{\mathbf{r}}\left(\mathbf{S}_{\mathbf{r}}^{\alpha} \times \mathbf{S}_{\mathbf{r}+\hat{\mathbf{x}}}^{\alpha} \cdot \hat{\mathbf{y}}-\mathbf{S}_{\mathbf{r}}^{\alpha} \times \mathbf{S}_{\mathbf{r}+\hat{\mathbf{y}}}^{\alpha} \cdot \hat{\mathbf{x}}\right),
\end{aligned}
$$

where $\mathbf{S}_{\mathbf{r}}^{\alpha}$ denotes a normalised spin at position $\mathbf{r}$ in either the top $(\alpha=T)$ or bottom $(\alpha=B)$ layer. For simplicity we assume $\mathbf{B}=B \hat{\mathbf{z}}$ to be aligned with the $z$-axis. The interlayer part is given by:

$$
H_{\text {inter }}=-J_{\mathrm{z}} \sum_{\mathbf{r}} \mathbf{S}_{\mathbf{r}}^{T} \cdot \mathbf{S}_{\mathbf{r}+\hat{\mathbf{z}}}^{B}
$$

We use Monte Carlo simulations to investigate the ground state of this model. In our Monte Carlo simulation a random configuration of spins is generated. In one Monte Carlo step we pick a 
random spin and propose a new vector its direction. This vector is chosen from a cap which size is chosen such that the acceptance rate in our Metropolis algorithm is $50 \%$. To reach the ground state and avoid getting trapped in local minima, we use simulated annealing. There we thermalise a system at a high temperature, far above the critical temperature, and subsequently lower the temperature stepwise until a temperature close to zero is reached. This proces is done multiple times. The simulations are started at a scaled temperature of $k_{\mathrm{B}} T / J_{\mathrm{xy}}=10$, where $k_{\mathrm{B}}$ is the Boltzman constant and $T$ is the temperature. At each temperature step the scaled temperature is lowered by a factor of 0.95 until a value of $k_{\mathrm{B}} T / J_{\mathrm{xy}}=0.01$ is reached.

\subsection{Results}

\subsubsection{Phases}

In this section we address the three types of phases in our simulations: the spiral state, skyrmion state and fully polarised state. In the spiral state the DMI and exchange dominate. The rotation direction of the spirals is determined by the DMI, where a positive DMI leads to clockwise rotating spirals, and thus a negative DMI to counterclockwise spirals. Furthermore, the wavelength is determined by the ratio of the DMI and intralayer exchange coupling. See Fig. 2.1 A for a snapshot of a z-projection of a spiral state. For an increased external magnetic field we find the skyrmion state. Skyrmions form in lattices and between the skyrmions spins point along the external magnetic field, as shown in Fig. 2.1 B. High external magnetic field can suppress the formation of skyrmions and subsequently systems containing a small number of skyrmions, without lattices structure, are found as well. The size of a skyrmion is determined by the ratio of the DMI and intralayer exchange coupling and is called the skyrmion pitch size $p$ which is given by $D / J_{\mathrm{xy}}=\tan (2 \pi / p)$. In the polarised state the external magnetic 
field dominates over the anisotropy and DMI and all the spins in the system point in the same direction as the external magnetic field as shown in Fig. 2.1 C.

We determine the skyrmion state from the winding number. The winding number (also called chirality or topological charge) is an integer which represents the number of times the spins enclose an unit sphere. The skyrmion state is recognised as the system with a nonzero winding number. In the continuum limit the winding number is expressed as:

$$
w=\frac{1}{4 \pi} \int d x d y \mathbf{m} \cdot\left(\frac{\partial \mathbf{m}}{\partial x} \times \frac{\partial \mathbf{m}}{\partial y}\right),
$$

where $x, y$ are the directions within the plane and $\mathbf{m}=\langle\mathbf{S}\rangle /|\langle\mathbf{S}\rangle|$ is the magnetisation direction in the system. In Fig. 2.1 we show the phase diagram, and typical spin configurations of the phases. Systems with a nonzero winding number possess skyrmions and thus are indicated as such in the phase diagram. In the phase diagram we plotted a contour plot of the total winding number of the system. In our systems the skyrmions have a winding number of positive one so the total winding number of a system is a measure for the total number of skyrmions in the system. In lattice systems, such as described in this paper, the winding number is continuous instead of integer. We find that in practice the winding number is a useful tool to distinguish the skyrmion phase from other magnetic phases.

Upon including the interlayer exchange, we see that in the synthetic ferromagnetic region there is no significant difference compared to the non-interacting picture as shown in figure Fig. 2.2. Looking at the synthetic antiferromagnetic region we see that the skyrmion pocket is situated at higher external magnetic field. This is explained as follows: the spiral state can adjust to the synthetic antiferromagnetic coupling by shifting the spirals in different layers half a period with respect to each other without increasing the 
energy. On the contrary, the polarised phase cannot adjust in a comparable way and needs a higher external magnetic field to adjust to the synthetic antiferromagnetic coupling. The skyrmion state is partly polarised while also having a lattice which can shift in respect to each other. Our simulations show that the lower bound of the skyrmion pocket rises considering the energetic advantage of the spiral state. The higher bound of the pocket rises as well because of the energy advantage of the skyrmion lattices over the polarised state originating from the shifting of the skyrmion lattices with respect to each other.

\subsubsection{Structural phase transition}

Taking a closer look at the synthetic antiferromagnetic skyrmion phases we notice that the lattice configurations change from a hexagonal lattice, Fig. 2.3 a), to a square one Fig. 2.3 c). We determine the lattice configurations of the skyrmions by looking at the reciprocal lattice which we obtain by taking a two dimensional Fourier transform of the configuration. A hexagonal lattice show six equally distributed first order peaks, as shown in Fig. 2.3 b), where a square lattice shows four Fig. $2.3 \mathrm{~d}$ ). The spiral phase is recognisable with having a zero winding number and two peaks with an angle of 180 degrees between each other. The fully polarised state has no nonzero winding number nor a nontrivial reciprocal lattice.

In Fig. 2.4 the phase diagram for different intra-layer exchange and external magnetic field is shown. It is visible that the skyrmion pocket location is dependent on the magnetic field in the antiferromagnetic intra-layer exchange region as already shown in Fig. 2.2. This leads to a larger spiral phase in the antiferromagnetic region. The hexagonal structural phase is dominant in the ferromagnetic part of the phase diagram and extends in the antiferromagnetic region: for a large antiferromagnetic intra-layer exchange the lattice configuration changes from hexagonal to square. This square 


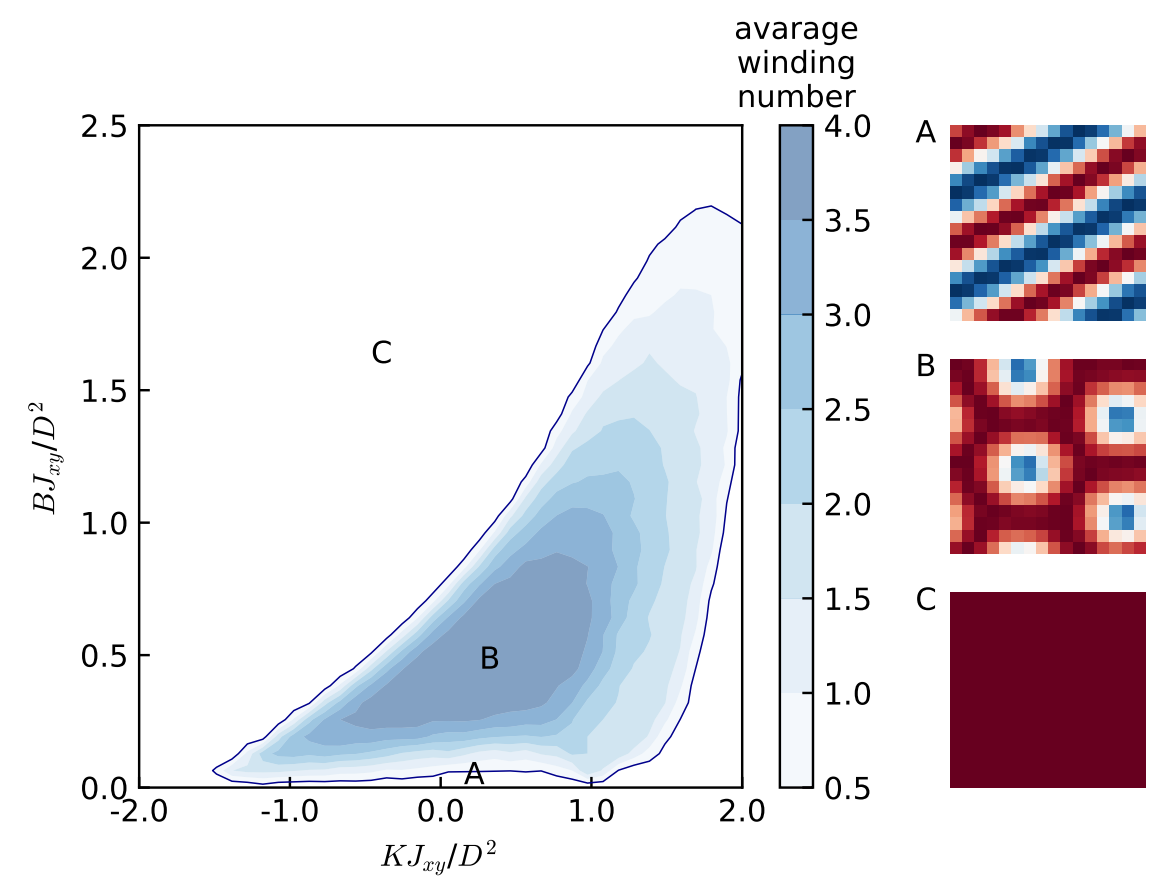

Figure 2.1: Phase diagram of the skyrmion pocket for $B J_{\mathrm{xy}} / D^{2}$ versus $K J_{\mathrm{xy}} / D^{2}$ with $J_{\mathrm{z}} / J_{\mathrm{xy}}=0$ at $k_{\mathrm{B}} T / J_{\mathrm{xy}}=0.01$. The colour scale depicts the winding number. The outer contour line is at winding number equals 0.5. A, B, C depicts the phases found on the given locations in the phase diagram, snapshots and phase diagram are from a $16 \times 16$ system with a pitch of $p=8$. The colour in the snapshots depicts the $z$-component of the spin using the colorbar in Fig. 2.3. 


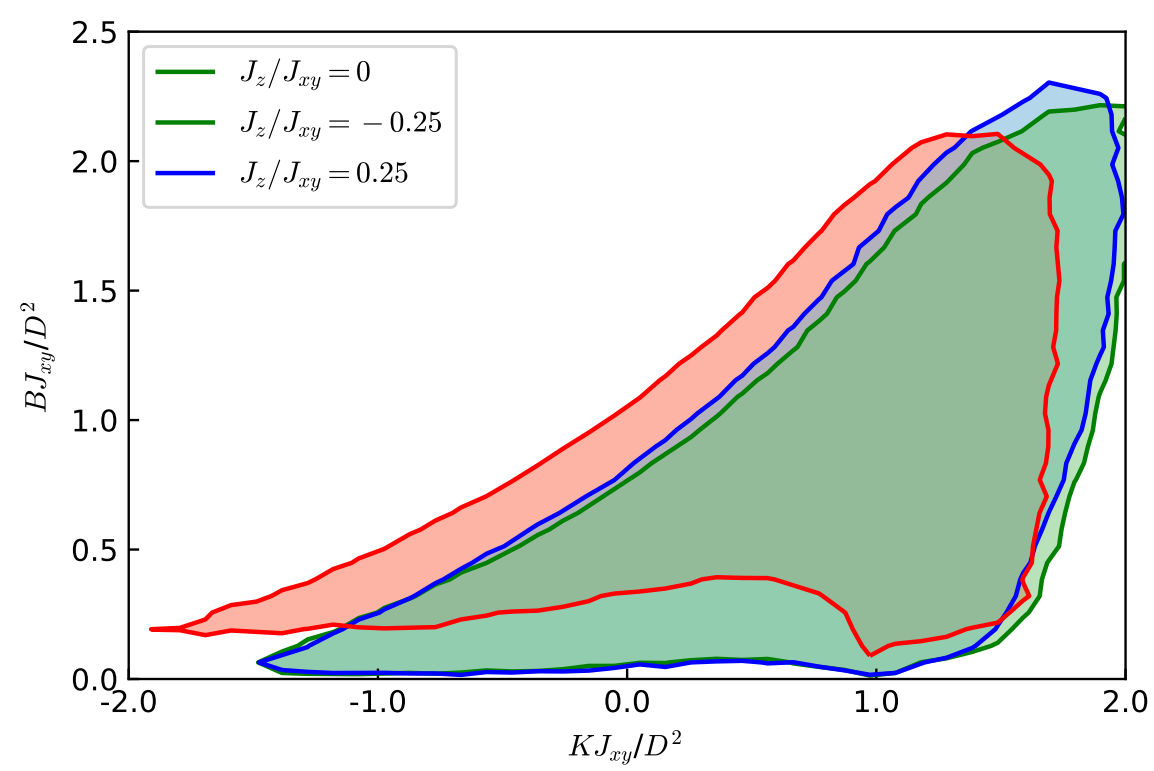

Figure 2.2: Phase diagram of the skyrmion pocket for $B J_{\mathrm{xy}} / D^{2}$ versus $K J_{\mathrm{xy}} / D^{2}$ at $k_{\mathrm{B}} T / J_{\mathrm{xy}}=0.01$ with $J_{\mathrm{z}} / J_{\mathrm{xy}}=0$ (blue), -0.25 (red) and 0.25 (green). The skyrmion pocket is drawn for systems with a total winding number $w>0.5$. It shows that the skyrmion pocket for $J_{\mathrm{z}} / J_{\mathrm{xy}}=0$, and 0.25 overlay, and that the skyrmion pocket for $J_{\mathrm{z}} / J_{\mathrm{xy}}=-0.25$ occurs for higher magnetic field. The simulated system had a system size of $16 \times 16$ spins with a pitch of $p=8$. 

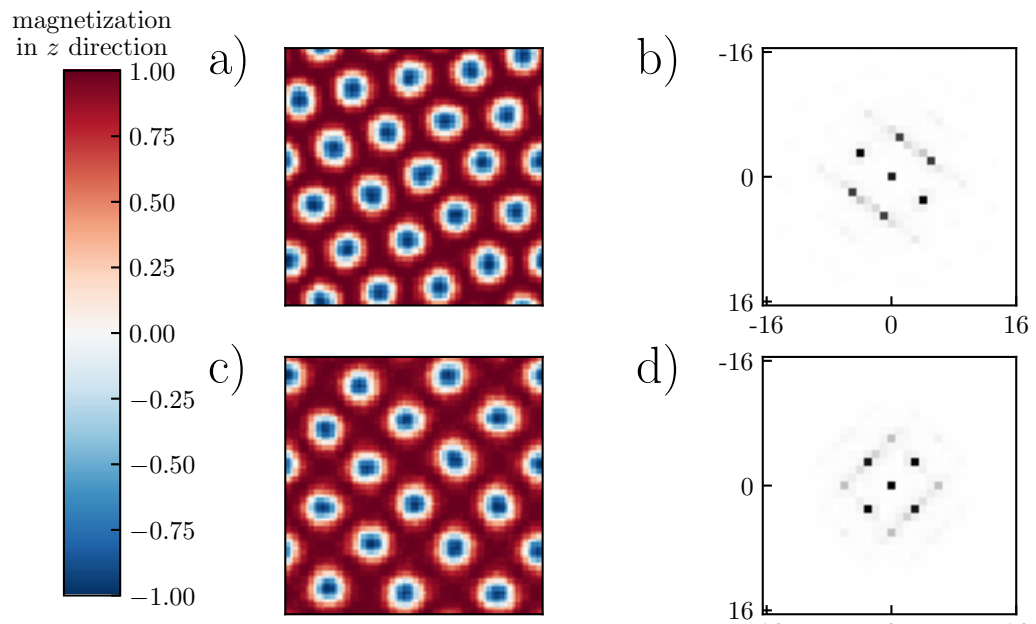

d)

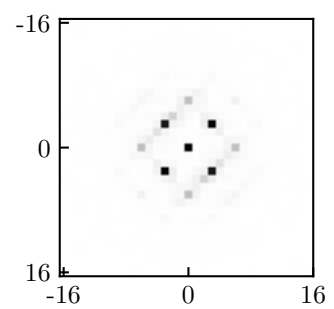

Figure 2.3: Spin configurations of a $64 \mathrm{x} 64, p=13, B J_{\mathrm{xy}} / D^{2}=$ $0.56, K J_{\mathrm{xy}} / D^{2}=0$ system at $k_{\mathrm{B}} T / J_{\mathrm{xy}}=0.01$. Showing a) hexagonal lattice configuration with $J_{\mathrm{z}} / J_{\mathrm{xy}}=-0.14$, b) the two dimensional Fourier transform of the hexagonal lattice configuration c) square lattice configuration with $J_{\mathrm{z}} / J_{\mathrm{xy}}=0.01$. d) the two dimensional Fourier transform of the square lattice configuration. From the Bragg peaks the lattice configuration is classified.

skyrmion lattice occurs only in the antiferromagnetic part.

\subsection{Discussion}

In this section, we turn to a physical interpretation of our results. For small skyrmions, skyrmions consisting of a small number of spins, the skyrmion lattice has a preferred orientation with respect to the underlying lattice, this can indicate that the skyrmion lattice is influenced by pinning to the underlying lattice. To test whether the square lattice configurations originate from this effect we increased the skyrmion pitch by lowering the DMI in our simulations. 


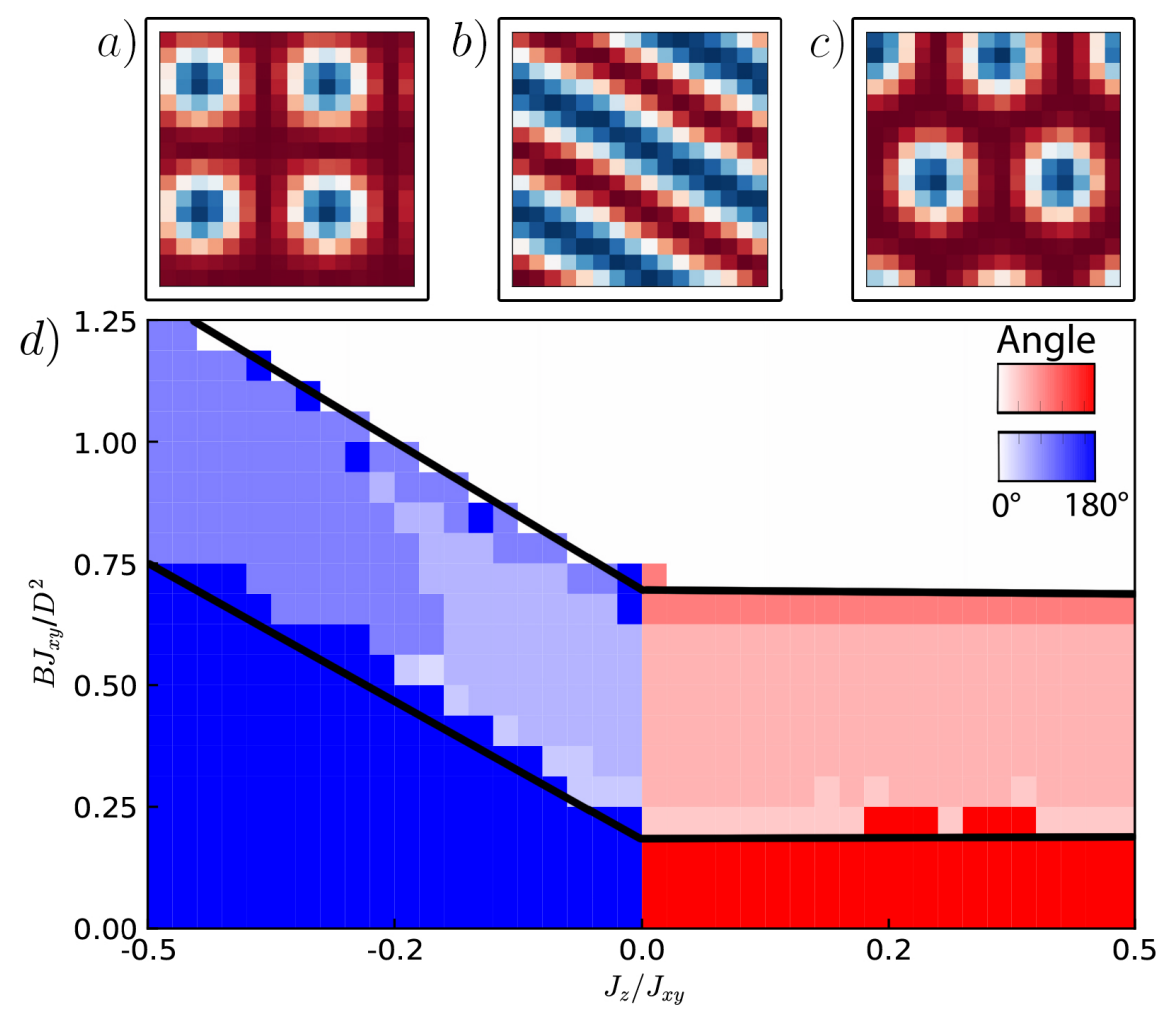

Figure 2.4: Spin configurations of a $16 \times 16$ system showing a) square lattice configuration, b) spiral configuration and c) hexagonal lattice configuration. The colour in the snapshots depicts the z-component of the spin using the colorbar in Fig. 3. d) Phase diagram of a two layer SAF for changing interlayer exchange $J_{\mathrm{z}} / J_{\mathrm{xy}}$ and magnetic field $B J_{\mathrm{xy}} / D^{2}$. The colour blue corresponds to antiferromagnetic ordering between the two layers, and red to ferromagnetic ordering. The boundary of the skyrmion phase is indicated by the black lines. The colour scale depicts the smallest angle found between two peaks in the 2D Fourier Transformation of the state. The striped phase b) gives an angle of $180^{\circ}$, where the square lattice configuration a) gives $90^{\circ}$ and the triangular lattice configuration c) $60^{\circ}$. This phase diagram is calculated for a $16 \times 16$ system with a pitch of $p=8$ and $K J_{\mathrm{xy}} / D^{2}=0$ at $k_{\mathrm{B}} T / J_{\mathrm{xy}}=0.01$. 
We found that the skyrmion lattices orient independently of the underlying lattice and that the skyrmion lattice still possesses the square configuration for increased skyrmion pitch. Moreover, the periodic boundary conditions do not seem to influence the found results. Changing the aspect ratio of our systems or changing the periodic boundary conditions to twisted boundary conditions leads to no significant change in the skyrmion lattice configurations.

The phase transition between the hexagonal and square skyrmion lattice can be explained as follows. The spin in the centre of the skyrmion points in the same direction in both layers, i.e. along the external magnetic field. Due to the antiferromagnetic interlayer exchange two skyrmions in different layers favour to be not on top of each other. Therefore, the skyrmion lattices are shifted relative to each other such that the skyrmions are positioned such that the spins in the different layers point oppositely. This implies that the skyrmion lattices in different layers are each other's dual lattice. The dual layer of a hexagonal lattice is a honeycomb lattice, and the dual lattice of square lattice is a square lattice again. It is not possible with equal skyrmion densities to form a honeycomb lattice in the dual lattice of the hexagonal lattice since the skyrmions will overlap. For a square lattice it is possible to position the skyrmions in their own dual lattice, which gives an energetic advantage over the triangular lattice. For a schematic display see Fig. 2.5. To test our interpretation we change the skyrmion density in one of the layers by altering the Dzyaloshinskii-Moriya interaction in that layer. By increasing the DMI, the skyrmion size decreases and the skyrmion density increases. Our interpretation suggests that the skyrmion lattice can have a honeycomb configuration in the layer with altered DMI because the smaller skyrmions do not overlap anymore. In Fig. 2.6 a snapshot is plotted of a spin configuration for parameters in the synthetic antiferromagnetic-square lattice regime but with different DMI for the two layers. Here the triangular lattice configuration manifests in the lower density layer and 
the honeycomb lattice in the higher density layer, confirming our interpretation of the structural phase transition.

As seen in Fig. 2.4, the structural phase transition occurs around $J_{\mathrm{z}} / J_{\mathrm{xy}}=-0.25$ for $p=8$. By looking at simulations with larger systems and skyrmion pitch sizes we noted that the phase transition occurs at different values of intra-layer exchange dependent on the pitch size of the skyrmions. For larger pitch size the phase transition is at higher intra-layer exchange, e.g. for skyrmion pitch $p=13$ the phase transition is around $J_{\mathrm{z}} / J_{\mathrm{xy}}=-0.02$. While our finding of the existence of the structural transition appears thus unchanged for larger system sizes, the precise value of $J_{\mathrm{z}} / J_{\mathrm{xy}}$ where the transition occurs is dependent on skyrmion size. Because of the excessive simulation time, we have not attempted finite-size scaling to determine the thermodynamic limit of the value of $J_{\mathrm{z}} / J_{\mathrm{xy}}$ where the transition occurs.

\subsection{Conclusion and Outlook}

In this article we studied skyrmions in synthetic antiferromagnets. We found that skyrmions still occur in bilayer synthetic antiferromagnets, but in the synthetic antiferromagnetic case a higher external magnetic field is needed than in the synthetic ferromagnetic case or the case without intralayer coupling. Skyrmion lattices in the synthetic antiferromagnetic case shift from a triangular to a square lattice for increasing interlayer coupling. Both the interlayer coupling and the external field have influence on the lattice geometry and both parameters are experimentally adjustable through either spacer thickness, external electric field, and through external magnetic field. As seen in Fig. 2.4, the phase transition between the hexagonal synthetic antiferromagnetic part and the square synthetic antiferromagnetic part has a magnetic field dependence which gives an opportunity for applications.

Different skyrmion lattices configurations such as the hexagonal- 


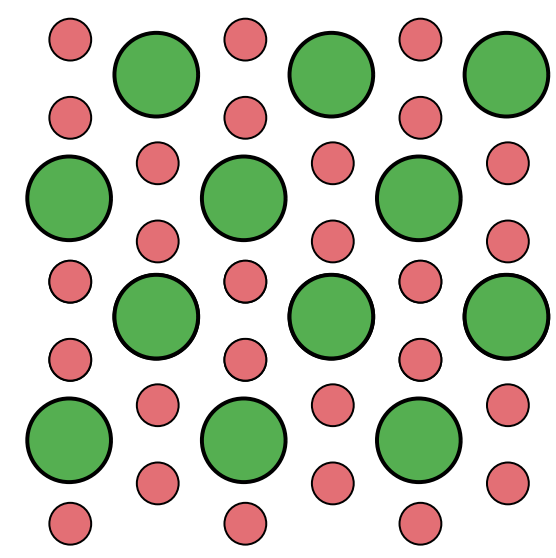

(a)

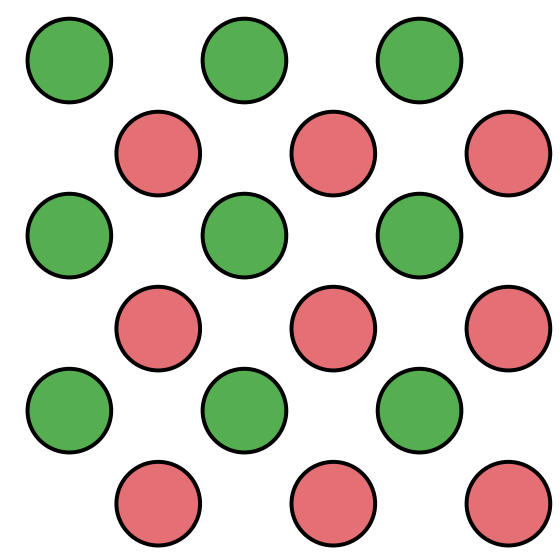

(b)

Figure 2.5: Schematic display of (a) a triangular lattice (green) together with its dual lattice (red) (b) a square lattice (green) with its dual lattice (red). The dual lattice of triangular lattice (a) has a higher density than the triangular lattice self, while the dual lattice of the square lattice (b) has an equal density. 

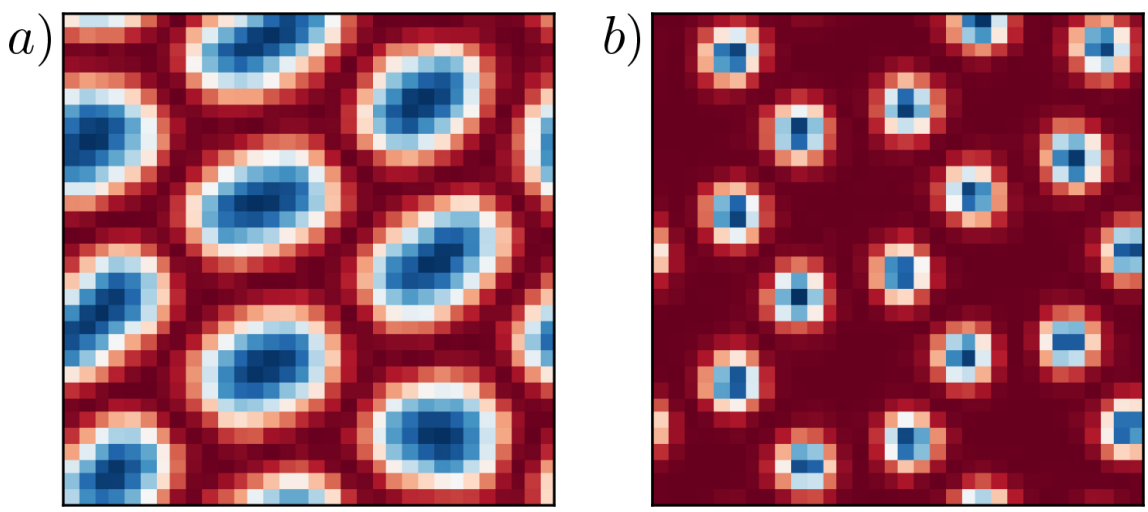

Figure 2.6: Snapshot of a spin configuration in both layers with $32 \mathrm{x} 32$ spins, $J_{z} / J_{\mathrm{xy}}=-0.20, B J_{\mathrm{xy}} / D^{2}=0.65, K J_{\mathrm{xy}} / D^{2}=0$ at $k_{\mathrm{B}} T / J_{\mathrm{xy}}=0.01$, the colour depicts the $z$-component of the spin (see colorbar Fig. 2.3). Layer a) has a pitch of $p=10$ and layer b) $p=6.25$. We see a triangular lattice in a) and a honeycomb lattice in b). 
honeycomb configurations mentioned in Section 2.4 could be explored further. For example, the phase diagram could be determined.

Experimental verification of triangular-honeycomb configurations can be obtained by multilayer systems with different DMI in both layers. This can be engineered by creating two layers with different compositions [77]. Direct observations of skyrmion lattices can be obtained by spin polarised scanning tunnelling microscopy or Lorentz transmission electron microscopy [78, 79]. The phase transition between the two lattice configurations is dependent on the ratio between the interlayer and intralayer exchange, and the ratio between the DMI and intralayer exchange. For a pitch size of $p=13$ the interlayer- intralayer exchange ratio is $J_{\mathrm{z}} / J_{\mathrm{xy}} \sim 0.007$. This value is experimentally achievable [65].

This work is part of the research programme Skyrmionics towards skyrmions for nanoelectronics, which is financed by the Netherlands Organisation for Scientific Research (NWO). 


\section{Layer effects on the mag- netic textures in mag- nets with local inversion asymmetry}

\subsection{Introduction}

The role of electronic devices in society is ever increasing, and there is a need to make them smaller and faster, whereas keeping a low-power consumption. However, the current technologies are reaching their limits since the information density cannot be increased much further. Therefore, new technologies need to be developed. One of the most promising new technologies for data processing and storage are magnetic systems with chiral textures such as chiral magnetic domain walls and skyrmions [37, 38, 8183]. Their chiral and topological properties make for sturdy textures which can become extremely small, this makes them suitable for applications. An example of such a design is the skyrmionracetrack memory, which is a promising route for fast and energy

\footnotetext{
${ }^{1}$ The contents of this chapter are based on the work of E. van Walsem, R.A. Duine and M.H.D. Guimarães [80]. RD and MHDG conceived the project, EvW carried out all computations and wrote the initial manuscript, MHDG designed Fig. 3.1 (a). All authors commented on the manuscript.
} 
efficient memory and processing devices [30, 33, 61].

(a)

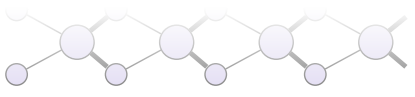

Layer 1

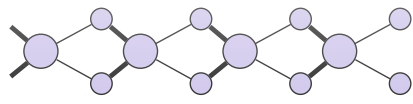

- $\longleftarrow$ Global Inversion symmetry

Layer 2

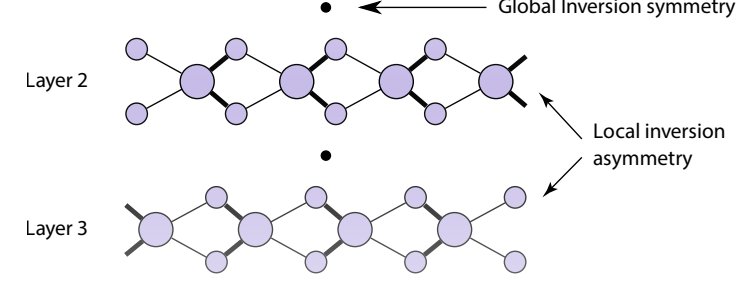

Layer 3
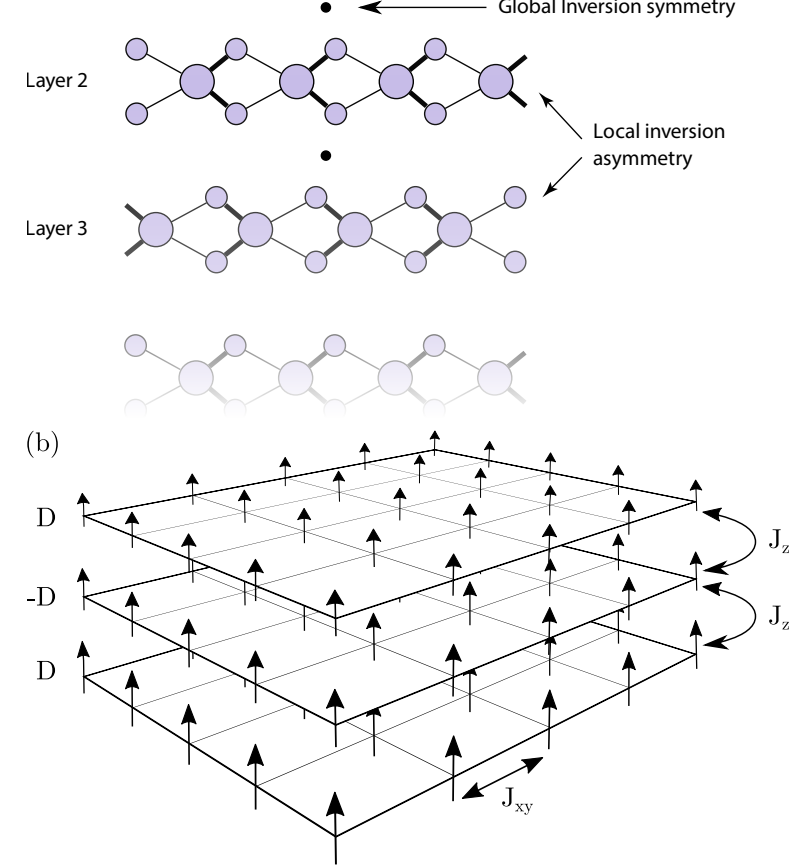

Figure 3.1: Schematics of (a) a bulk crystal with a global inversion symmetry whereas showing local inversion asymmetry and (b) the model used in our simulations. Here, the arrows are Heisenberg spins and can rotate freely in three dimensions, $J_{\mathrm{xy}}$ is the intralayer coupling, $J_{\mathrm{z}}$ is the interlayer coupling, and $D$ is the DMI.

At the moment, a plethora of systems is known to host skyrmionic textures, such as the chiral magnet MnSi which hosts 
Layer 1

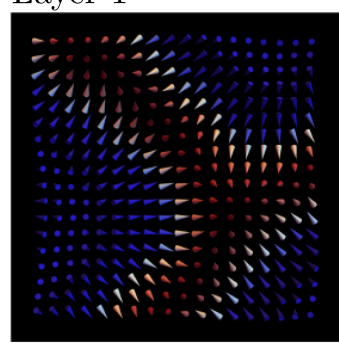

Layer 2

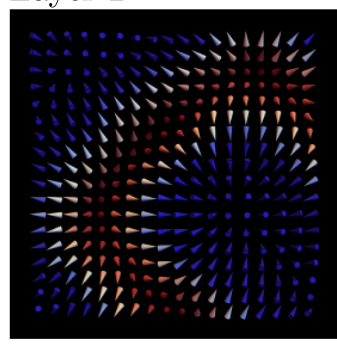

Layer 3

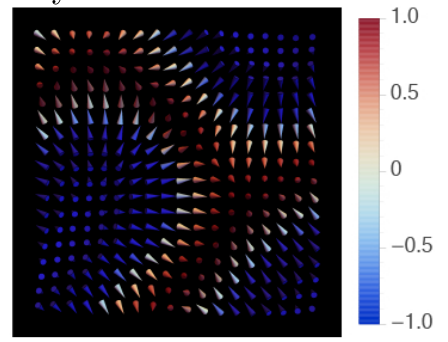

Figure 3.2: Snapshot of a $32 \times 32$ cut out of a three layer $128 \times 128$ spinsystem with $J_{\mathrm{z}} / J_{\mathrm{xy}}=0.10$ and $D / J_{\mathrm{xy}}=0.50$. The color red indicates that a spin is pointing upwards and blue downwards. Spin spirals are clearly visible, and the sign of the DMI has a clear effect on the turning sense (counterclockwise for layers 1 and 3 and clockwise for layer 2).

so-called Bloch skyrmions and ultra thin ferromagnetic films which typically host Néel skyrmions [41, 42, 45, 55-60]. These thin magnetic films are formed by stacking multiple layers of different metals, and the order of these layers determine their magnetic properties. Furthermore, a new class of suitable materials is emerging: the Van der Waals crystals. These crystals consists of two dimensional layers of one atom thick, stacked on top of each other via Van der Waals bonds [84, 85]. Two-dimensional layers can be exfoliated from bulk materials, such as graphite, hexagonal boron nitride and $\mathrm{CrI}_{3}[84,86,87]$. Because of the freedom to stack different kinds of materials, the end product is tunable and can be formed such that the desired properties are present in the end product with virtually no strain since the weak interlayer bonds make them less sensitive to lattice mismatch problems.

In this article, we demonstrate how a local DMI arising from a local inversion asymmetry can give rise to chiral structures even 
in materials with global inversion symmetry. DMI is an interaction formed when strong spin-orbit coupling and broken inversion symmetry are present. An example of such symmetry breaking is the interface between two different materials, such as Co and $\mathrm{Pt}$ $[34,35]$. The DMI is also referred to as antisymmetric exchange since the interaction picks up a minus sign when exchanging two spins. Because of this property, the interaction leads to chiral magnetic textures. One example of chiral magnetic textures is spirals with a preferred turning sense (either clockwise (CW) or counterclockwise (CCW)) which are formed by DMI in the absence of fields and anisotropies. Here we note that a local DMI can arise in crystal possessing global inversion symmetry, but which show a local inversion asymmetry (as drawn in Fig. 3.1). This allows for a DMI term to be non zero locally whereas averaging out when the complete infinite crystal is taken into account This is analogous to the "hidden spin polarization" effect that occurs because of local inversion symmetry breaking and was elucidated in Ref. [88] and experimentally verified in Van der Waals crystals [89-92]. We are interested in magnets where this local DMI has an alternating nature of its sign in subsequent layers. Especially, we are interested in materials with (anti)ferromagnetic coupling between the layers. To comply with this condition, Van der Waals crystals need to have bulk inversion symmetry, layer inversion asymmetry, magnetism, and high spin-orbit coupling. An example of a Van der Waals material meeting these criteria is $\mathrm{Fe}_{3} \mathrm{GeTe}_{2}$ [85], which belongs to the space-group $P 6_{3} / m m c$ [93] in its bulk form and point-group $D_{3 h}$ in its monolayer form [94]. The condition of local inversion asymmetry in a globally inversion symmetric system can also be obtained in sputtered metallic thin films, such as $\mathrm{Ta} / \mathrm{Co} / \mathrm{Pt} / \mathrm{Co} / \mathrm{Ta}$ systems making it even easier to perform such DMI engineering [95].

In this article, we discuss how a locally nonzero DMI influences magnetic textures. We show what different textures form and find that these chiral textures occurring in the system are influenced by 
the stacking of the layers. The resulting spin spiral wavelength and their turning sense are affected by the interlayer coupling relative to the DMI. Furthermore, we find that the number of layers influences the wavelength of the spin spirals in the system, and an evenodd effect is found for the number of layers in the system. The tunability of the spin spirals suggests that skyrmions will also be tunable in their size. The tunability of the spin spiral wavelength and turning sense demonstrate the potential of DMI engineering for new magnetic devices. Moreover, since spin spiral systems can develop skyrmions upon applied magnetic fields, our results also serve as a basis for the design of skyrmionic devices.

The remainder of this paper is organized as follows. In Section 3.2 we discuss our model and how the simulations are performed. After this we show results of our simulations in Section 3.3 where we focus on the phase diagram, wavelength and turning sense found in the system with an odd number of layers. This is compared in Section 3.3.4 to systems with an even number of layers. Finally, we conclude with an outlook in Section 3.4.

\subsection{Model and Method}

To model a stack of coupled ferromagnetic layers, we describe them with a classical Heisenberg spin model. Each layer is modeled by equally spaced spins on a square lattice. This is performed for simplicity and is a decent approximation since we are modeling temperatures far below the Curie temperature and are interested in smooth textures, such as spin spirals. The layers are placed right on top of each other as shown in Fig. 3.1 (b), and each layer has an alternating sign for the DMI strength. We assume that the leading interactions within the layers are ferromagnetic nearestneighbor exchange, and DMI. The leading interaction between the layers is assumed to be nearest-neighbor exchange varying from the ferromagnetic to the antiferromagnetic regime. We note that these 
conditions are met for various Van der Waals crystals as well as for metallic thin film heterostructures with Ruderman-Kittel-KasuyaYosida (RKKY) coupled layers.

We describe the Hamiltonian $H$ with separate terms for the intra- and interlayer terms,

$$
H=H_{\text {intra }}+H_{\text {inter }} .
$$

The interactions within the layers are expressed as follows:

$$
\begin{gathered}
H_{\text {intra }}=-J_{\mathrm{xy}} \sum_{\alpha=1}^{N} \sum_{\mathbf{r}} \mathbf{S}_{\mathbf{r}}^{\alpha} \cdot\left(\mathbf{S}_{\mathbf{r}+\hat{\mathbf{x}}}^{\alpha}+\mathbf{S}_{\mathbf{r}+\hat{\mathbf{y}}}^{\alpha}\right) \\
+\sum_{\alpha=1}^{N}(-1)^{\alpha-1} D \sum_{\mathbf{r}}\left(\mathbf{S}_{\mathbf{r}}^{\alpha} \times \mathbf{S}_{\mathbf{r}+\hat{\mathbf{x}}}^{\alpha} \cdot \hat{\mathbf{y}}-\mathbf{S}_{\mathbf{r}}^{\alpha} \times \mathbf{S}_{\mathbf{r}+\hat{\mathbf{y}}}^{\alpha} \cdot \hat{\mathbf{x}}\right),
\end{gathered}
$$

where $J_{\mathrm{xy}}$ is the intralayer coupling, $D$ is the Dzyaloshinskii-Moriya interaction, $\mathbf{S}_{\mathbf{r}}^{\alpha}$ is the spin at position $\mathbf{r}$ in layer $\alpha=1,2, \ldots$ and $\hat{\mathbf{x}}$ and $\hat{\mathbf{y}}$ are the unit vectors in the $x$ and $y$ directions, respectively. The number of layers is denoted with $N$ and the $(-1)^{\alpha}$ term regulates the alternating DMI sign in the system. The interactions between the layers are described by:

$$
H_{\text {inter }}=-J_{\mathrm{z}} \sum_{\alpha=1}^{N} \sum_{\mathbf{r}} \mathbf{S}_{\mathbf{r}}^{\alpha} \cdot \mathbf{S}_{\mathbf{r}}^{\alpha+1},
$$

where $J_{\mathrm{z}}$ is the intralayer coupling.

To investigate the ground state of this model, we use Monte Carlo simulations. We begin the simulation by taking a random spin configuration at a high temperature. Then, we use the Metropolis algorithm to thermalize the system [52, 53]. This algorithm picks a random spin and proposes a new semi random 
direction. This new direction is such that the average acceptance ratio is $50 \%$, which is determined from the energy difference between the new and the old spin configuration: $\Delta E$. The acceptance probability $\mathcal{P}$ is then $\mathcal{P}=\exp \left(\Delta E / k_{\mathrm{B}} T\right)$ if $\Delta E<0$ or $\mathcal{P}=1$ in all other cases. Finally, we accept or reject this new direction with a probability of 0.5 . When the system is fully thermalized, we decrease the temperature and repeat this thermalization and lowering of the temperature until the temperature gets close to zero and approaches the ground state. We start our simulations at $k_{\mathrm{B}} T / J_{\mathrm{xy}}=10$, where $k_{\mathrm{B}}$ is the Boltzman constant and $T$ is the temperature. To this end, the temperature is lowered by a factor of 0.95 until $k_{\mathrm{B}} T / J_{\mathrm{xy}}=0.01$.

\subsection{Results for odd number of layers}

For low temperatures, we expect that magnetic textures will form in the ferromagnet. The DMI leads to the formation of a chiral spiral inside the layer and, depending on the sign of the DMI, the turning sense of the spiral should be different in each subsequent layer. The size of the spiral should be influenced by the relative strength between the DMI and the intralayer coupling terms $\left(D / J_{\mathrm{xy}}\right)$. We performed simulations for varying parameters of DMI $\left(D / J_{\mathrm{xy}}\right)$ and interlayer coupling $\left(J_{\mathrm{z}} / J_{\mathrm{xy}}\right)$ and systems with sizes varying between $32 \times 32$ spins and $256 \times 256$ spins. We find that the systems thermalize and form a stable state where a clear chiral spiral pattern is visible. In Fig. 3.2, we show snapshots of such a system with chiral spirals. We preformed simulations for a varying number of layers, and, in this part of the article, we will discuss systems with an odd number of layers. The even number of layer systems are discussed later in Section 3.3.4. 


\subsubsection{Phases and Phase Diagram}

The magnetic structures in our systems will be determined by the competition among the three terms in the model: the intralayer coupling, which favors the alignment of the spins inside the layer, the DMI, which leads to a chiral spiral inside of the layer, and the interlayer coupling, which favors the (anti-)alignment of the spins between the layers.

Our simulations show six prominent magnetic phases. The first two phases are the fully polarized phases where the interlayer exchange is dominant, i.e., $\left|J_{\mathrm{z}}\right| / J_{\mathrm{xy}} \gg D / J_{\mathrm{xy}}$. Here, no magnetic structure appears, all spins are always aligned inside the layer. For a negative interlayer exchange $J_{z}$, the layers are antiferromagnetically (AFM) oriented with respect to each other, this is in contrast with a positive interlayer exchange where the layers are oriented ferromagnetically (FM). A cartoon of this is shown in Fig. 3.3 (a) Ia for $J_{\mathrm{z}}>0$ and Ib for $J_{\mathrm{z}}<0$. The next two phases, phases IIa and IIb, are obtained by increasing the DMI and magnetic spirals start to form. Here, the DMI term is non-negligible when compared to $J_{z}$. We have observed this for all finite non zero values of $D / J_{\mathrm{xy}}$. Since the interlayer coupling is still fairly large compared to the DMI in this phase, the system behaves, such as the complete system possesses a single "net" DMI value, and all spirals have the same turning sense as is shown in Fig. 3.3 (a) IIa and IIb. Important to note, here, is that the system we consider in this section is still inversion asymmetric due to the odd number of layers which does allow for a net DMI to be present. Furthermore, the sign of the interlayer coupling $J_{\mathrm{z}}$ determines the relative orientation of the layers, here, as well. The difference is shown in Fig. 3.3 IIa for FM and IIb for AFM orientation between the layers. The last two phases are where the DMI is dominant and since the sign of the DMI is alternating, the turning sense of the spirals is also alternating in subsequent layers. As is drawn in Fig. 3.3 (a) IIIa and IIIb. 
(a)

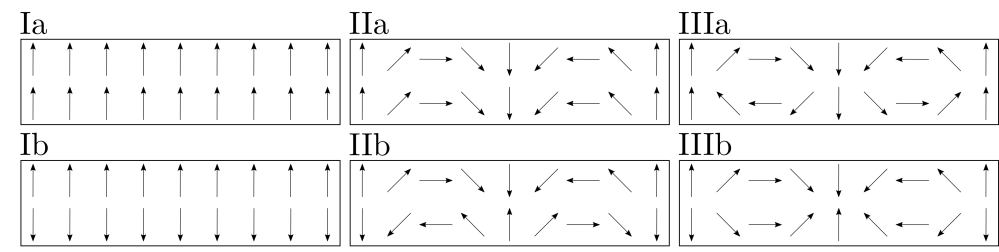

(b)

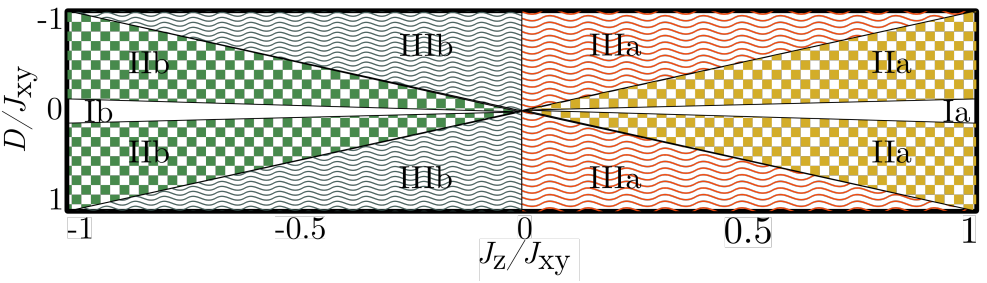

Figure 3.3: (a) Cartoon of two coupled spin layers corresponding to the phases described above. (b) Phase diagram of an odd multilayer system. Phases Ia and Ib correspond to the ferromagnetic (FM) and antiferromagnetic (AFM) polarized phases respectively, phases IIa (FM) and IIb (AFM) to the interlayer coupling dominant phase and phases IIIa (FM) and IIIb (AFM) to the DMI dominant phase. The phase diagram is determined with help from the second layer of a five layers system with $256 \times 256$ spins with a DMI between 1 and -1 and a $J_{z} / J_{x y}$ between 1 and -1 .

Here, the interlayer exchange is only present to regulate the relative orientation of the layers. The snapshot in Fig. 3.2 is taken in the DMI dominant phase (IIIa), a counterclockwise turning sense is visible in layers 1 and 3 and a clockwise turning sense in layer 2. In Fig. 3.3 (b), we plot the phase diagram where these three phases occur for different values of DMI and interlayer coupling.

In Fig. 3.4, we show the average turning sense for a system with five layers. We determined this turning sense by looking at the average value of turning direction between two neighboring spins $C_{\mathrm{TS}}$, which we defined by

$$
C_{\mathrm{TS}}=\operatorname{Sgn}\left[\left(\mathbf{S}_{\mathbf{r}} \times \mathbf{S}_{\mathbf{r}+\hat{\mathbf{x}}}\right) \cdot \hat{y}\right],
$$


Chapter 3. Layer effects on the magnetic textures in magnets with local

\section{Layer 1}

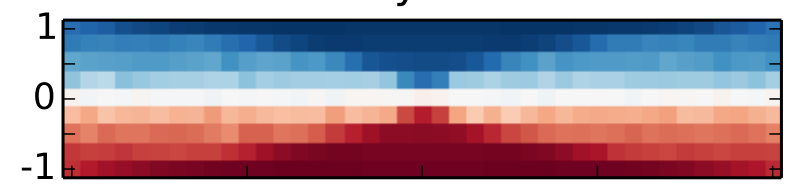

Layer 2

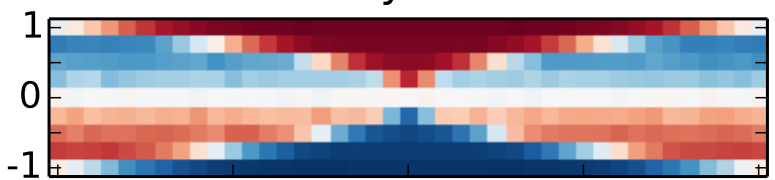

Layer 3

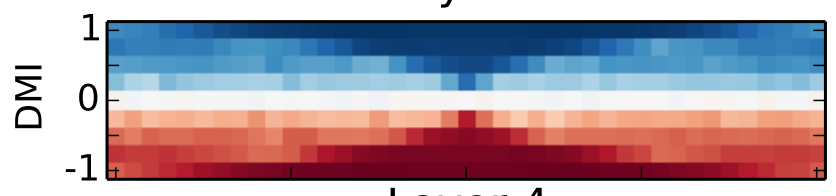

Layer 4

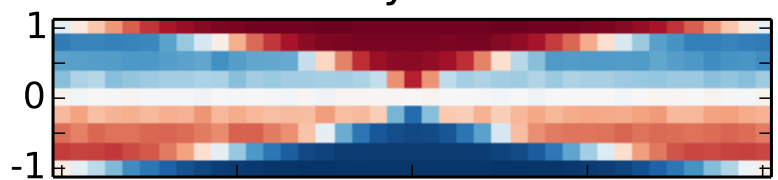

Layer 5

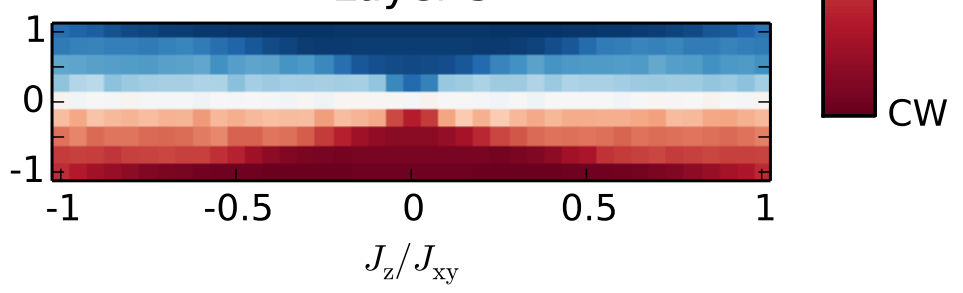

Figure 3.4: Average turning sense in each layer. To calculate this average turning sense each spin is given a value of \pm 1 corresponding to the orientation with respect to their neighboring spin, finally an average of all these values is calculated. A value of +1 corresponds to a clockwise (CW) turning sense and -1 to an counter clockwise (CCW) turning sense. This is plotted for five layers of $256 \times 256$ spins with a DMI between 1 and -1 , and a $J_{\mathrm{z}} / J_{\mathrm{xy}}$ between 1 and -1 . 
where our reference vector $\hat{y}=(0,1,0)$, is the unit vector along $y$. A positive $C_{\mathrm{TS}}$ corresponds to a $\mathrm{CW}$ orientation between the spins, and negative $C_{\mathrm{TS}}$ corresponds to a $\mathrm{CCW}$ orientation. The total average value of all turning directions gives an indication of how the spiral is oriented in the layer on average. In Fig. 3.4, the fully polarized phases Ia and Ib are clearly visible as the white region where $D / J_{\mathrm{xy}}=0$ and no turning sense is present. For increased DMI, a non zero average turning sense is visible, corresponding to phases IIa and IIb. In each subsequent layer, the turning sense has the same direction. Increasing the DMI even further, we find phases IIIa and IIb, and this is clearly visible in Fig. 3.4. There is a stronger turning sense, and each subsequent layer switches the turning sense following the sign of the DMI. In layers 2 and 4 , a right (left) turning sense is found for a negative (positive) value of $D / J_{\mathrm{xy}}$ instead of a left (right) turning sense.

The only difference between ferromagnetic $\left(J_{\mathrm{z}}>0\right)$ and antiferromagnetic $\left(J_{\mathrm{z}}<0\right)$ interlayer couplings is is the relative orientation of the layers. For the rest, all plots in Fig. 3.4 (b) are symmetric around $J_{\mathrm{z}} / J_{\mathrm{xy}}$. We can explain this from looking at Fig. 3.4 (a) IIa and IIb, here, we see that the ferromagnetic coupled layers have aligned spirals, and the antiferromagnetic coupled layers have anti aligned spirals. This difference can be described as a phase shift between the spirals of half a wavelength. Furthermore, for Fig. 3.4 (a) IIIa, the spins pointing perpendicular to the layer are aligned between the layers, but due to the alternating nature of the DMI, the parallel pointing spins are anti aligned. In Fig. 3.4 (a) IIIb the opposite is true: The perpendicular spins are anti aligned, but the parallel spins are not. This contrast makes that there is no noticeable energy difference between the ferromagnetic and the antiferromagnetic cases. 


\subsubsection{Wave vector}

In this section, we will focus on the wave vector of the spirals found in phase IIa/b and IIIa/b. The wave vector is defined by the number of cycles a spiral forms per spin and is determined by the ratio of DMI and intralayer exchange coupling. A higher ratio leads to a shorter spiral period and thus a larger wave vector. We expect that the competition between the DMI and the interlayer coupling has significant effects on the spirals since two coupled spirals with different turning senses cannot be coupled such that all spins are aligned. In Fig. 3.3 (a) III, it is visible that the interlayer coupling between the two spirals gives a different energy contribution per spin: a favorable energy contribution for the vertically aligned spins and an unfavorable one for the horizontal anti aligned spins. It is impossible to shift the spirals relative to each other such that the interlayer coupling is favorable for all spins. The wave vector will be the largest where the interlayer coupling is not present. Here, with $J_{\mathrm{z}} / J_{\mathrm{xy}}=0$ the wave vector is the same as a single layer system and goes to zero where the interlayer coupling is much larger than the DMI.

In Fig. 3.5, we show the wave vector for constant DMI, $D / J_{\mathrm{xy}}$ ranging from 0 to 1 , and varying interlayer exchange. A big variation in the wave vector is found between small and large interlayer exchanges. The wave vector for the large interlayer exchange is around one-fifth the size of the wave vector for the small interlayer exchange. The drop off between these two cases corresponds to the phase transition in the phase diagram between phases IIa/b and IIIa/b. Thus, we see that phase IIa/b has a larger wave vector than phase IIIa/b. Also, here, there is no clear distinction visible between the ferromagnetic and the antiferromagnetic interlayer couplings. To examine the distance between the different cases of $D / J_{\mathrm{xy}}$ in Fig. 3.5, we plot the wave-vector dependence on the DMI for different values of interlayer coupling $J_{\mathrm{z}} / J_{\mathrm{xy}}$ in Fig. 3.6. Here, 
we see that the relation between DMI and wave vector without any interlayer coupling is linear, but for increasing interlayer coupling, this linearity is not found since the wave vector is dependent on the different phases of the system.

Since the wave vector is influenced by finite-size effects, we used a finite-size scaling to determine the true wave vector. For this, simulations were preformed for systems with system size $64 \times 64$, $128 \times 128$ and 256x256 and we extrapolated the wave vector linearly in $1 / L$. An example of a plot with different system sizes and the resulting true wave vector is shown in Fig. 3.8. In the inset, we show one of the data fits we used to determine the wave vector. The wave vector is determined as the average distance to the origin for all pixels in a two dimensional Fourier transform that are more then five standard deviations above the mean of all pixels in a layer, divided by the system size.

\subsubsection{Influence of the number of layers}

We investigated the wave vector for systems with three, four, and five layers. In this section, we are focusing on systems with an odd number of layers only. See Fig. 3.7 for a comparison between two systems with three and five layers. It is visible that the drop off is larger for a larger number of layers. Examining the ratio between the long wavelength in the DMI dominant phase and the shorter wavelength in the interlayer coupling dominant phase we find two effects. First, this ratio is roughly 3 for a system with three layers and around 5 for a system with five layers. Our hypothesis is that the sum of the DMI divided by the number of layers gives a net DMI for strong interlayer coupling. At $J_{\mathrm{z}} / J_{\mathrm{xy}}=0$, the layers are completely decoupled and behave as individual layers. Here, the net DMI in each individual layer is exactly $D / J_{\mathrm{xy}}$. As we increase $J_{z}$, the interlayer coupling leads to a "dilution" of the DMI, and, at very large interlayer coupling, the system behaves as a system with a single DMI value, given by the sum of the DMI in each individual 


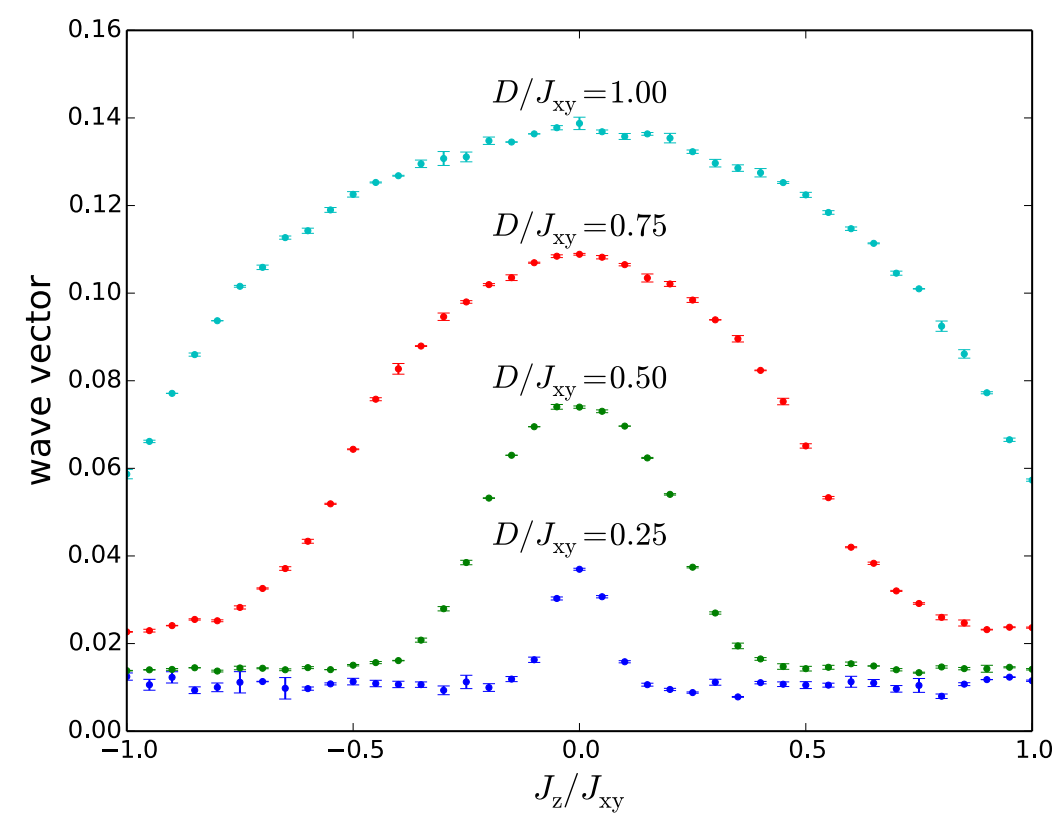

Figure 3.5: Wave vector for various values of DMI plotted for a range of interlayer coupling for a five layer system. A small wave vector corresponds to a larger spiral wave length. 


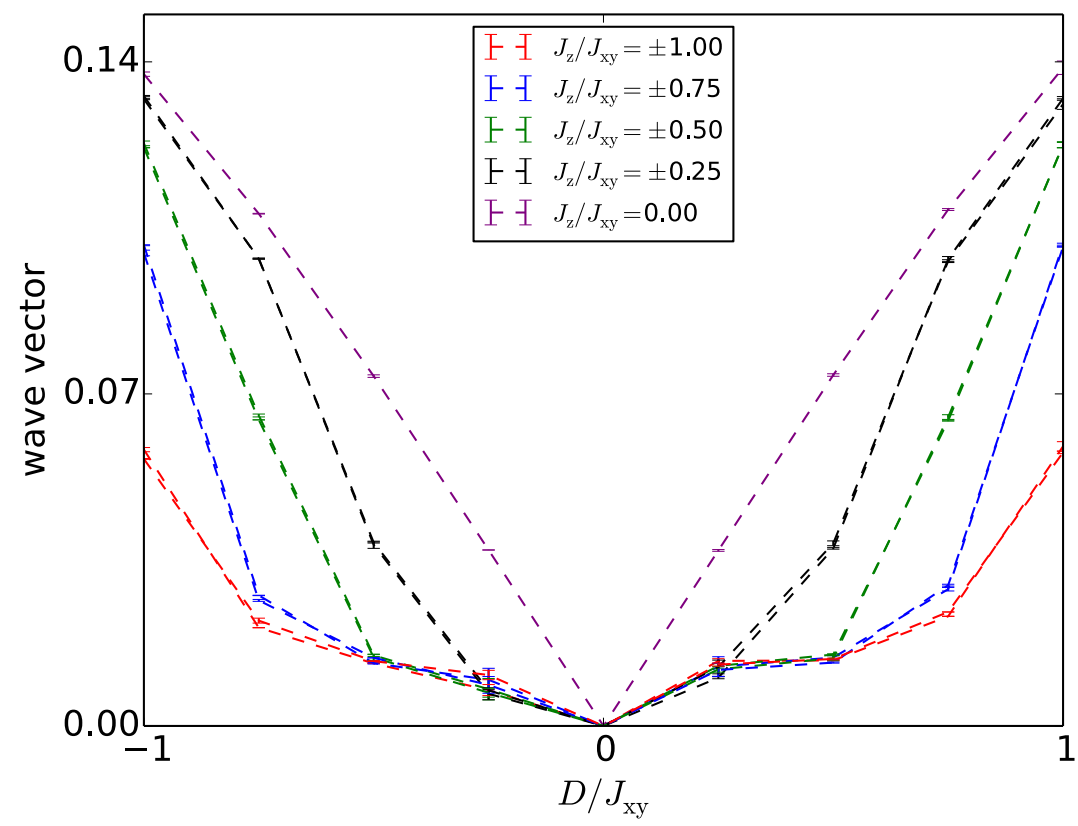

Figure 3.6: Wave vector for various values of interlayer coupling plotted for a range of DMI for a five layer system corresponding to Fig. 3.5. It is visible that the linear behavior of the wave vector is affected by the interlayer coupling. 
Chapter 3. Layer effects on the magnetic textures in magnets with local

\begin{tabular}{l|llllll}
\hline$D / J_{\text {xy }}$ & $\mathbf{- 0 . 7 5}$ & $\mathbf{- 0 . 5 0}$ & $\mathbf{- 0 . 2 5}$ & $\mathbf{0 . 2 5}$ & $\mathbf{0 . 5 0}$ & $\mathbf{0 . 7 5}$ \\
\hline Layer 1 & 2.88 & 2.96 & 2.66 & 2.82 & 3.14 & 2.88 \\
Layer 2 & 3.00 & 3.04 & 2.69 & 2.75 & 3.24 & 3.01 \\
Layer 3 & 2.91 & 2.95 & 2.60 & 2.74 & 3.13 & 2.89 \\
\hline
\end{tabular}

Table 3.1: Table of the ratio between the wave number in the DMI dominating phase IIIa/b and the phase IIa/b in a three-layer system. Columns are for $D / J_{\mathrm{xy}}$ and rows are for the different layers in a system. This is for a three layer system. The ratio is determined for $J_{z} / J_{\mathrm{xy}}= \pm 1$ and $J_{\mathrm{z}} / J_{\mathrm{xy}}=0$. The results for $D / J_{\mathrm{xy}}= \pm 1$ are omitted in this table because this strong the interlayer coupling must be stronger than the intralayer coupling to reach phase IIa/b.

layer divided by the number of layers. As the number of layers increases, the DMI gets divided by an increasing number, Therefore leading to a decrease in the net total DMI and a reduction of the observed wave vector. Because of the alternating DMI the total sum of the DMI is $D$ leading to a net DMI $D_{\text {net }}=D / 3$ for three layers and $D_{\text {net }}=D / 5$ for five layers. This is only a rough approximation which works within $10 \%$ of the ratio. Second, the precise ratio of the layers themselves shows a pattern. There is a symmetry, the two outermost layers have the same ratio, whereas the inner layer has a different ratio. For example, in a five layered system, the second and fourth layers have the same ratio, and there is a symmetry around the middle layer. This hints to a richer structure between the layers. We show the ratios in Table 3.1 and Table 3.2 for which we used a system with $J_{\mathrm{z}} / J_{\mathrm{xy}}= \pm 1$ for the wave vector of an interlayer coupling dominating phase and the values at $J_{\mathrm{z}} / J_{\mathrm{xy}}=0$ as the DMI dominating phase. 


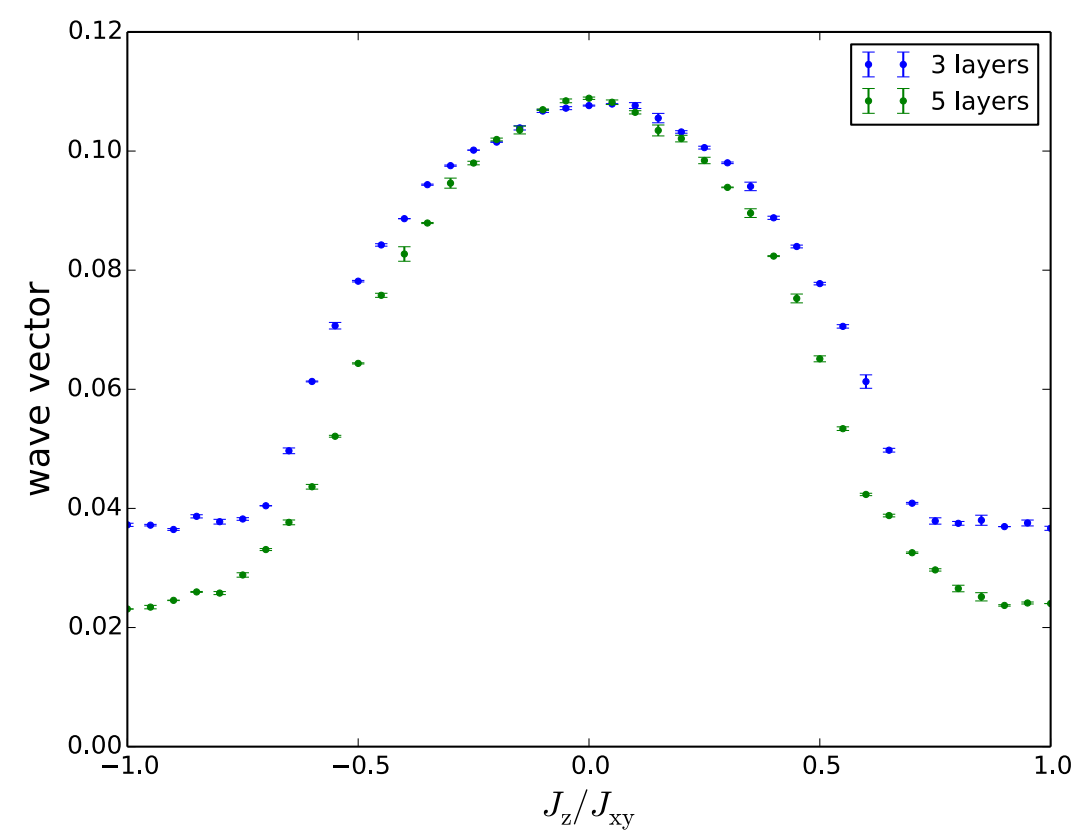

Figure 3.7: Comparison of the wave vectors of a system with three layers and a system with five layers. Both layers are plotted for a DMI of $D / J_{\mathrm{xy}}=0.75$. A drop off between 2.6 and 3.2 is visible in the three-layered system and between 3.3 and 5.3 in the five-layered system. 
Chapter 3. Layer effects on the magnetic textures in magnets with local

\begin{tabular}{l|llllll}
\hline$D / J_{\text {xy }}$ & $\mathbf{- 0 . 7 5}$ & $\mathbf{- 0 . 5 0}$ & $\mathbf{- 0 . 2 5}$ & $\mathbf{0 . 2 5}$ & $\mathbf{0 . 5 0}$ & $\mathbf{0 . 7 5}$ \\
\hline Layer 1 & 4.47 & 4.65 & 3.37 & 3.25 & 5.21 & 4.57 \\
Layer 2 & 4.65 & 4.77 & 3.38 & 3.34 & 5.31 & 4.60 \\
Layer 3 & 4.56 & 4.68 & 3.40 & 3.29 & 5.28 & 4.59 \\
Layer 4 & 4.66 & 4.77 & 3.37 & 3.27 & 5.34 & 4.63 \\
Layer 5 & 4.51 & 4.64 & 3.44 & 3.24 & 5.21 & 4.51 \\
\hline
\end{tabular}

Table 3.2: Table of the ratio between the wave number in the DMI dominating phase IIIa/b and the phase IIa/b in a five layer system. Columns are for $D / J_{\mathrm{xy}}$, and rows are for the different layers in a system. This is for a five layer system. Our approximation of $D_{\text {net }}$ breaks down at $D / J_{\text {xy }}= \pm 0.25$ where the ratios are much smaller. The ratio is determined for $J_{\mathrm{z}} / J_{\mathrm{xy}}= \pm 1$ and $J_{\mathrm{z}} / J_{\mathrm{xy}}=0$. The results for $D / J_{\mathrm{xy}}= \pm 1$ are omitted in this table because this strong the interlayer coupling must be stronger than the intralayer coupling to reach phase IIa/b.

\subsubsection{Results for even number of layers}

When a thin ferromagnet with local inversion asymmetry has an even number of layers, the sum of the total DMI will be zero, i.e., $D_{\text {net }}$ will be zero. Thus, in the ferromagnetic dominant phase, we expect to observe no spin spirals. This is, indeed, the case. In Fig. 3.8, we plot the wave vector of a system with four layers. In the DMI dominant phase, we still find spin spirals with a wave vector of 0.07 inverse lattice spacings, which is comparable to the odd layered case. However, in the interlayer coupling dominate phase, the wave vector drops to the predicted 0 . Unfortunately, the finitesize scaling breaks down in this regime and gives noisy results, and to prevent confusion, we decided not to show the noisy results. To ensure that the wave vector is, indeed, zero, we examined qualitatively all snapshots of the systems where $\left|J_{z} / J_{x y}\right|<0.5$. Here, we, indeed, see that no spin spirals are present in the interlayer 
dominating region. However, we observe localized spin textures, a region where the spins are oriented differently from the rest of the polarized system. These are formed by thermal fluctuations around the nucleation temperature and are stabilized by the DMI which is still present. This is a metastable state, but due to the higher number of local minima in the phase space, our Monte Carlo simulations do not reach the global minimum. By comparing these textures to uniform magnetized textures, we found that these artifacts have a higher energy and are, thus, not representative for the ground state. The error bars for these structure also indicate the volatility of these textures and indicate that they are not the ground state.

\subsection{Conclusion, discussion and outlook}

In conclusion, we presented results on the behavior of spin textures in ferromagnets with a local inversion asymmetry between the layers which leads to alternating DMI for consecutive layers. We found strong effects for the chiral spiral textures originating from the DMI. Furthermore, we were able to distinguish three different phases: the polarized phase, the DMI-dominant phase, and the interlayer coupling-dominant phase. The interlayer couplingdominant phase has shown different behaviors for the number of layers in a system by influencing the wavelength of the spirals and shown a strong difference between odd and even numbers of layers in a system.

In this paper, we used a minimal model. Future research can be focused to include additional magnetic effects known to influence textures, such as anisotropy, dipole-dipole interactions, and an external magnetic field. Altought the finite-size scaling of our results gives a good indication of the expected wave number and a maximum system size of $256 \times 256$ spins is still efficient to simulate, more certainty in the wave number is possible with larger system 


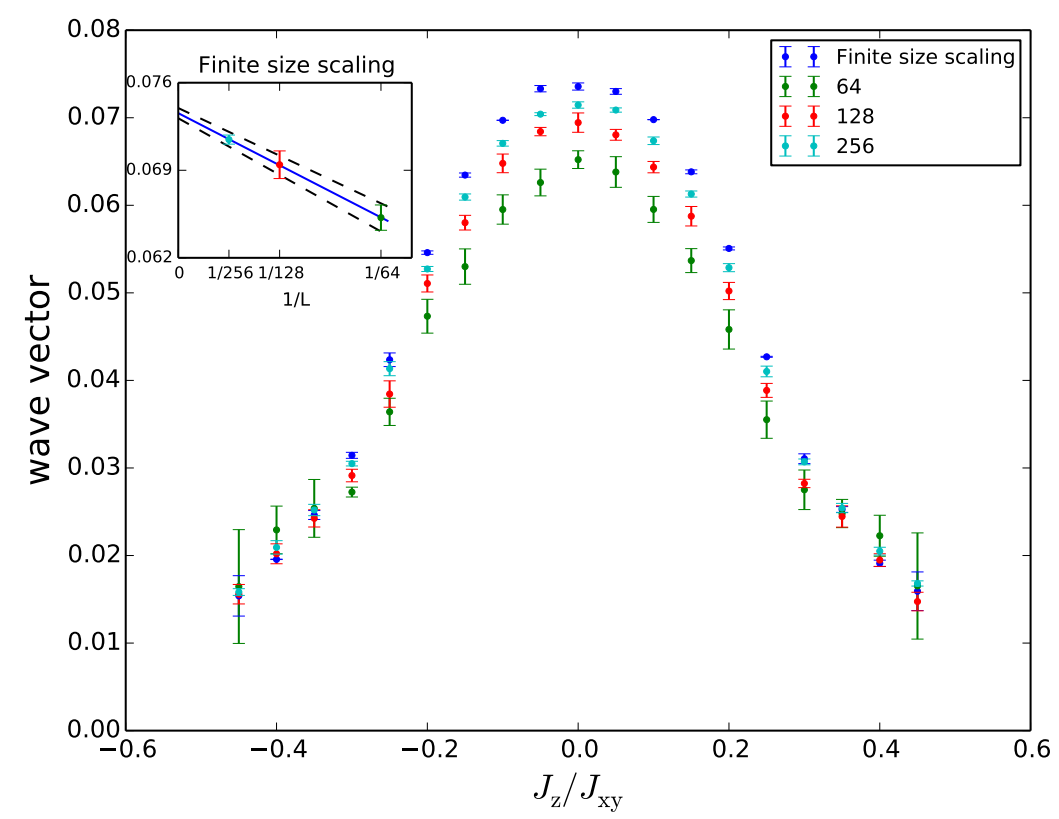

Figure 3.8: Wave vector plotted against interlayer coupling $J_{\mathrm{z}} / J_{\mathrm{xy}}$ for a system with four layers and a DMI of $D / J_{\mathrm{xy}}=0.50$. Different system sizes $64 \times 64,128 \times 128$ and $256 \times 256$ are plotted, and a finitesize scaling made with a linear fit in $1 / L$ is added. The inset shows the finite-size scaling for $J_{\mathrm{z}} / J_{\mathrm{xy}}=0$. Here, we only show the results smaller than $\left|J_{\mathrm{z}} / J_{\mathrm{xy}}\right|<0.5$ since the finite-size scaling did not work above this value. 
sizes. More computations can also be used to test our hypothesis of the net DMI that occurs in coupled layers. A higher number of layers should continue the trend reported in this paper. Furthermore, the phase diagram in this paper gives a direction to where the different phases occur, a more detailed version can be achieved by simulations for more parameters. The small energy scales and high number of meta stable states of chiral systems makes it difficult for Monte Carlo simulations to reach the ground state. By preforming multiple simulations and finding comparable spin configurations we are convinced that our results are close to the ground state and are representative of the ground state itself.

Our results shown here demonstrate that rich magnetic structures can be obtained through local DMI engineering. First, as a useful method to measure the Dzyaloshinskii-Moriya interaction in Van der Waals ferromagnets or multilayer ferromagnets with a broken local inversion symmetry. When the number of layers and coupling between the layers is known, the DMI can be determined by looking at the spiral wave vector in the upper layer. Second, possible applications that need the ability to tune spiral wave length or even completely turn off spin spirals. This can be achieved by changing the coupling between the layers, e.g., through applying pressure a Van der Waals material $[20,96]$ or by changing the spacing layer in a RKKY-coupled metallic stack. Third, our results serve as a prelude to investigating skyrmions in Van der Waals magnets. Moreover, an addition of an external magnetic field should lead to the formation of skyrmions.

R.D. is member of the D-ITP consortium, a program of the Dutch Organization for Scientific Research (NWO) that is funded by the Dutch Ministry of Education, Culture and Science(OCW). This project has received funding from the European Research Council (ERC) under the European Union's Horizon 2020 research and innovation programme (grant agreement No. 725509). This work is part of the research program Skyrmionics - towards 
skyrmions for nanoelectronics, which is financed by the Dutch Research Council (NWO). M.H.D.G. acknowledges the financial support of NWO (VENI 15093). 


\section{$4 \quad$ Collective modes of skyrmions in synthetic antiferromagnets ${ }^{1}$}

\subsection{Introduction}

The coupling of magnetic layers containing skyrmions leads to exciting new insights. For example: we have shown in Chapter 2 that layers containing skyrmion lattices change their lattice structure upon tuning the interlayer coupling strength. Moreover, skyrmion tracks benefit from coupling two layers, since the Magnus effect, where a skyrmion deviates from its trajectory depending on its winding number, is negated by coupling two skyrmions with opposite winding number such that the two Magnus effects are nullified [70, 97]. Furthermore, it has also been shown that coupling multiple layers stabilize skyrmions altogether [60]. Crucial for further research into the effect of coupling layers with skyrmions is to detect the skyrmions present in them. Various methods to detect skyrmions are known, such as topological hall effect measurements[58, 98], x-ray microscopy [45, 58, 99], magnetic force microscopy [58, 100], magneto-optical Kerr effect (MOKE) mi-

\footnotetext{
${ }^{1}$ The contents of this chapter are based on the results of a project in the Theory for technology course of the Theoretical Physics master at Utrecht University. It is written together with Joren Harms who was actively involved in the analytical calculations and Patrick van Dieten who was actively involved in the numerical magnon mode calculations.
} 
croscopy [101], Lorentz transmission electron microscopy (LTEM) $[41,102,103]$ and microwave response measured by a ferromagnetic resonance (FMR) spectrometer [104-106]. An FMR measurement is especially attractive as it is relatively simple and its theoretical understanding requires the computation of the collective modes of the system. Furthermore, on the basis of these collective modes, skyrmions can be used as an oscillator to generate microwave radiation giving rise to applications. In this chapter we focus on the detection of skyrmions by a FMR spectrometer. We theoretically predict the response of a skyrmion by looking at its frequency. This has been shown before for one layer [107] and has been proven useful for detecting skyrmions in Ref $[108,109]$. However, both have not been done for coupled systems of skyrmions. In this chapter we present the characteristics of the eigenfrequencies of two coupled skyrmions. We determined these frequencies using two methods. An analytic method where we minimize the action for our system, and a numerical method where we diagonalize the hamiltonian on a ground state determined by Monte Carlo simulations. We compare both methods and give some theoretical guidance on how to experimentally detect skyrmions in bilayers in the future.

\subsubsection{General description of the system}

In this chapter we consider two coupled magnetic layers. The magnetic layers are described by three dimensional Heisenberg spins arranged on a square lattice. Within this magnetic layer, we choose the interactions between the spins such that skyrmions are formed. For this we need the following interactions: a ferromagnetic interaction originating from exchange coupling, a chiral component such as strain in triangular materials or the Dzyaloshinksii Moriya interaction (DMI), and an easy axis anisotropy to fix the preferred axis for the spins to align with or a magnetic field to fix the preferred direction. Between the layers the spins are coupled through an exchange interaction which can be either ferromagnetic 
or antiferromagnetic. We choose the interactions such that precisely one skyrmion per layer is formed with equal winding number 1. The magnetic layers have open boundaries and therefore both skyrmions are repulsive from the boundaries and will be positioned in the center of their layer. Because of the absence of currents the position of the skyrmion is fixed.

Our system can be described by a hamiltonian, we do this in two parts: the intralayer part which describes all the above mentioned factors inside of the layer and the interlayer part which describes the coupling of the layers trough an exchange interaction. The intralayer Hamiltonian is expressed as:

$$
\begin{aligned}
H_{\text {intra }}= & -J_{\mathrm{xy}} \sum_{\alpha \in\{T, B\}} \sum_{\mathbf{r}} \mathbf{S}_{\mathbf{r}}^{\alpha} \cdot\left(\mathbf{S}_{\mathbf{r}+\hat{\mathbf{x}}}^{\alpha}+\mathbf{S}_{\mathbf{r}+\hat{\mathbf{y}}}^{\alpha}\right) \\
& +K \sum_{\alpha \in\{T, B\}} \sum_{\mathbf{r}}\left(\mathbf{S}_{\mathbf{r}}^{\alpha} \cdot \hat{\mathbf{z}}\right)^{2}-\mathbf{B} \cdot \sum_{\alpha \in\{T, B\}} \sum_{\mathbf{r}} \mathbf{S}_{\mathbf{r}}^{\alpha} \\
& -D \sum_{\alpha \in\{T, B\}} \sum_{\mathbf{r}}\left(\mathbf{S}_{\mathbf{r}}^{\alpha} \times \mathbf{S}_{\mathbf{r}+\hat{\mathbf{x}}}^{\alpha} \cdot \hat{\mathbf{y}}-\mathbf{S}_{\mathbf{r}}^{\alpha} \times \mathbf{S}_{\mathbf{r}+\hat{\mathbf{y}}}^{\alpha} \cdot \hat{\mathbf{x}}\right),
\end{aligned}
$$

where $\mathbf{S}_{\mathbf{r}}^{\alpha}$ is a classical Heisenberg spin at position $\mathbf{r}$ in layer $\alpha$ (top, T, or bottom, B). $J_{\mathrm{xy}}$ is the interlayer coupling, $K$ the anisotropy, B the external magnetic field and $D$ the Dzyaloshinskii-Moriya interaction. The Hamiltonian that describes the coupling of the layers is given by:

$$
H_{\text {inter }}=-J_{\mathbf{z}} \sum_{\mathbf{r}} \mathbf{S}_{\mathbf{r}}^{T} \cdot \mathbf{S}_{\mathbf{r}+\hat{\mathbf{z}}}^{B}
$$

where $J_{\mathrm{z}}$ is the interlayer exchange coupling. The total hamiltonian is thus:

$$
H=H_{\text {intra }}+H_{\text {inter }} .
$$


Examples of materials described by these hamiltonians are Synthetic Antiferromagnets (SAFs). In SAFs two thin film magnetic multilayers are coupled trough a metallic spacer. By varying the thickness of the spacer layer the coupling strength is regulated, and can even change sign.

\subsection{Analytical method}

In this section we will start with an action describing the system which we will minimize to find the equations of motion of the system. From there we are able to find the eigenfrequencies of the bilayer skyrmions.

The action of the system can be written as the action of the separate layers, and the interaction between those layers:

$$
\mathcal{S}=\mathcal{S}_{\text {top }}+\mathcal{S}_{\text {bottom }}+\mathcal{S}_{\text {int }}
$$

In this chapter we study the small coupling region. In this region the skyrmions only interact with spin waves within their own layer. Therefore we can use the results from Psaroudaki et al. to describe the top and bottom layers independently [110]. In their paper they describe the interaction of a skyrmion with spin waves by using a mass term $M$ as:

$$
\mathcal{S}_{\text {mass }}=\int d \tau \frac{1}{2} M \dot{R}^{2}
$$

where $R$ is the position of the center of mass of the skyrmion in its own layer. In this chapter we do not calculate the mass term $M$, but calculations for this are found in [110]. The Magnus effect on the skyrmion can be described as:

$$
\mathcal{S}_{\text {magnus }}=Q \frac{\beta_{\text {eff }}}{2} \epsilon_{i j} R^{i} \dot{R}^{j}
$$

where $\beta_{\text {eff }}=8 \pi S, \epsilon_{i j}$ the Levi-Civita symbol and $Q$ the topological charge or the winding number of the skyrmions. In this chapter 
we are only interested in skyrmions with a charge $Q=1$. In our system we have closed boundary conditions. To describe the repulsion of the skyrmions by the boundary we add an effective quadratic potential:

$$
V(R)=\frac{K}{2} R^{2}=\frac{K}{2}\left(R_{\mathrm{x}}^{2}+R_{\mathrm{y}}^{2}\right) .
$$

This will pull the skyrmion towards the center of the system with strength $K$.

\subsubsection{Ferromagnetic interaction}

The interaction between the layers is due to either a ferromagnetic exchange coupling or an anti-ferromagnetic one. In this subsection we will focus on the ferromagnetic exchange coupling first and the antiferromagnetic exchange coupling will be treated in the next subsection.

The interaction action, $\mathcal{S}_{\text {int }}$, in terms of magnetization $\Omega_{\mathrm{T} / \mathrm{B}}$ is described by:

$$
\mathcal{S}_{\text {int }}=\rho_{\text {int }} \int d \tau d \mathbf{x} \boldsymbol{\Omega}_{\mathrm{T}}\left(\mathbf{x}-\mathbf{R}_{\mathrm{T}}(\tau)\right) \cdot \boldsymbol{\Omega}_{\mathrm{B}}\left(\mathbf{x}-\mathbf{R}_{\mathrm{B}}(\tau)\right),
$$

where $\mathbf{R}_{\mathrm{T} / \mathrm{B}}$ is the position of the center of the skyrmion in either the top (T) or bottom (B) layer. We can rewrite this by introducing two new parameters defined by the sum and difference of the skyrmion centers:

$$
\begin{aligned}
& \mathbf{R}(\tau)=\frac{1}{2}\left(\mathbf{R}_{\mathrm{A}}(\tau)+\mathbf{R}_{\mathrm{B}}(\tau)\right) \\
& \mathbf{r}(\tau)=\frac{1}{2}\left(\mathbf{R}_{\mathrm{A}}(\tau)-\mathbf{R}_{\mathrm{B}}(\tau)\right) .
\end{aligned}
$$

Implementing this in the action Eq. (4.8) and assuming both skyrmions are placed on top of each other, since they are both positioned in the middle of their layer due to the quadratic potential, 
we can assume $|\mathbf{r}| \ll 1$. Therefore, the action can be expressed as:

$$
\begin{aligned}
\mathcal{S}_{\mathrm{int}} \simeq-\rho_{\mathrm{int}} \int d \tau d \mathbf{x} & {\left[\boldsymbol{\Omega}_{\mathrm{A}}-\partial_{\alpha} \boldsymbol{\Omega}_{\mathrm{A}} \mathbf{r}^{\alpha}+\frac{1}{2}\left(\partial_{\alpha} \partial_{\beta} \boldsymbol{\Omega}_{\mathrm{A}} \mathbf{r}^{\alpha} \mathbf{r}^{\beta}\right)\right] \times } \\
& {\left[\boldsymbol{\Omega}_{\mathrm{B}}+\partial_{\alpha} \boldsymbol{\Omega}_{\mathrm{B}} \mathbf{r}^{\alpha}+\frac{1}{2}\left(\partial_{\alpha} \partial_{\beta} \boldsymbol{\Omega}_{\mathrm{B}} \mathbf{r}^{\alpha} \mathbf{r}^{\beta}\right)\right] . }
\end{aligned}
$$

By expanding this expression and some algebra we end up with:

$$
\mathcal{S}_{\text {int }}=E-\rho_{\text {int }} \int d \tau d \mathbf{x}\left(\partial_{\alpha} \Omega_{\mathrm{A}} \cdot \partial_{\beta} \boldsymbol{\Omega}_{\mathrm{B}}\right) \mathbf{r}^{\alpha} \mathbf{r}^{\beta}+\mathcal{O}\left(\mathbf{r}^{3}\right)
$$

where we need to note that the first term, $E$, contains contributions that do not depend on the skyrmion position but is not necessarily zero.

Combining the four terms described above we end up with the following effective action:

$$
\begin{aligned}
\mathcal{S}_{\text {eff }}\left[R_{\mathrm{A}}, R_{\mathrm{B}}\right]=\int d \tau\{ & \frac{1}{2} M\left(\dot{R}_{\mathrm{A}}^{2}+\dot{R}_{\mathrm{B}}^{2}\right) \\
& +\frac{\beta_{\text {eff }}}{2} \epsilon_{i j} R_{\mathrm{A}}^{i} \dot{R}_{\mathrm{A}}^{j} \\
& +\frac{\beta_{\text {eff }}}{2} \epsilon_{i j} R_{\mathrm{B}}^{i} \dot{R}_{\mathrm{B}}^{j} \\
& -\frac{K}{2}\left(R_{\mathrm{A}}^{2}+R_{\mathrm{B}}^{2}\right) \\
& \left.-\frac{\alpha}{2}\left(R_{\mathrm{A}}-R_{\mathrm{B}}\right)^{2}\right\},
\end{aligned}
$$

where we defined $\alpha=\rho_{\text {int }} \int d \mathbf{x}\left(\partial_{\alpha} \Omega_{\mathrm{A}} \cdot \partial_{\beta} \Omega_{\mathrm{B}}\right)$ from Eq. (4.11).

The skyrmion configuration $\Omega$ can be described following Bogdanov et al. [111]:

$$
\hat{\mathbf{n}}_{\mathbf{0}}=\sin \theta_{0} \cos \phi_{0} \hat{\rho}+\sin \theta_{0} \sin \phi_{0} \hat{\phi}+\cos \theta_{0} \hat{z},
$$


where we used cylindrical coordinates $\rho, \theta$ and $\phi, \theta_{0}$ is the polar angle and $\phi_{0}$ the angle in the azimuthal direction. We determine the value of $\theta_{0}$ and $\phi_{0}$ by minimizing the energy: a continuous version of Eq. (4.3):

$$
\begin{array}{r}
E[\mathbf{\Omega}(\mathbf{x})]=\int d \mathbf{x}\left[-\frac{J_{\mathrm{xy}}}{2} \boldsymbol{\Omega} \cdot \nabla^{2} \boldsymbol{\Omega}+\frac{C}{2} \boldsymbol{\Omega} \cdot(\nabla \times \boldsymbol{\Omega})+K\left(1-\Omega_{z}^{2}\right)\right. \\
\left.+\mu_{0} H M\left(1-\Omega_{z}\right)-\mu_{0} M \boldsymbol{\Omega} \cdot \mathbf{H}_{\mathbf{d}}\right] .
\end{array}
$$

By parametrizing by Eq. (4.13) and integrating out $\phi$ and $z$ we end up with an energy density which we can minimize for $\theta_{0}$ and $\phi_{0}$. For a thin film $\phi_{0}=0$. Finally, we end up with the following differential equation for $\theta_{0}(\rho)$ :

$\frac{\partial^{2} \theta_{0}(\rho)}{\partial \rho^{2}}+\frac{1}{\rho} \frac{\partial \theta_{0}(\rho)}{\partial \rho}-\frac{\sin \left(2 \theta_{0}(\rho)\right)}{2 \rho^{2}}+\frac{\sin \left(\theta_{0}(\rho)\right)^{2}}{\rho}-B J_{\mathrm{xy}} / D^{2} \frac{\sin \left(\theta_{0}(\rho)\right)}{2}=0$,

which we can solve for different values of magnetic field $B J_{\mathrm{xy}} / D^{2}$. We show some examples of skyrmion profiles $\theta_{0}(\rho)$ in Fig. 4.1.

Using this we can write the coupling $\alpha$ as:

$$
\begin{aligned}
\alpha & =\int_{0}^{\infty} d \rho \int_{0}^{2 \pi} d \phi \rho \cdot\left(\partial_{i} \hat{\mathbf{n}}_{\mathbf{0}}\right)^{2} \\
& \simeq \pi \int_{0}^{\infty} d \rho\left\{\rho \cdot\left(\partial_{\rho} \theta_{0}\right)^{2}+\frac{\sin ^{2} \theta_{0}}{\rho}\right\} .
\end{aligned}
$$

In Fig. 4.2 we plotted the coupling for different skyrmion configurations with different values of external magnetic field $\frac{B J_{\mathrm{xy}}}{D^{2}}$.

Using the principle of least action we derive the equations of motion for our effective action $\mathcal{S}_{\text {eff }}$. This is expressed as follows:

$$
M\left(\begin{array}{l}
\ddot{R}_{\mathrm{A}} \\
\ddot{R}_{\mathrm{B}}
\end{array}\right)=\left(\begin{array}{cc}
-K+\beta_{\mathrm{eff}} \epsilon_{i j} \partial_{\tau}-\alpha & \alpha \\
\alpha & -K+\beta_{\mathrm{eff}} \epsilon_{i j} \partial_{\tau}-\alpha
\end{array}\right)\left(\begin{array}{l}
R_{\mathrm{A}} \\
R_{\mathrm{B}}
\end{array}\right),
$$




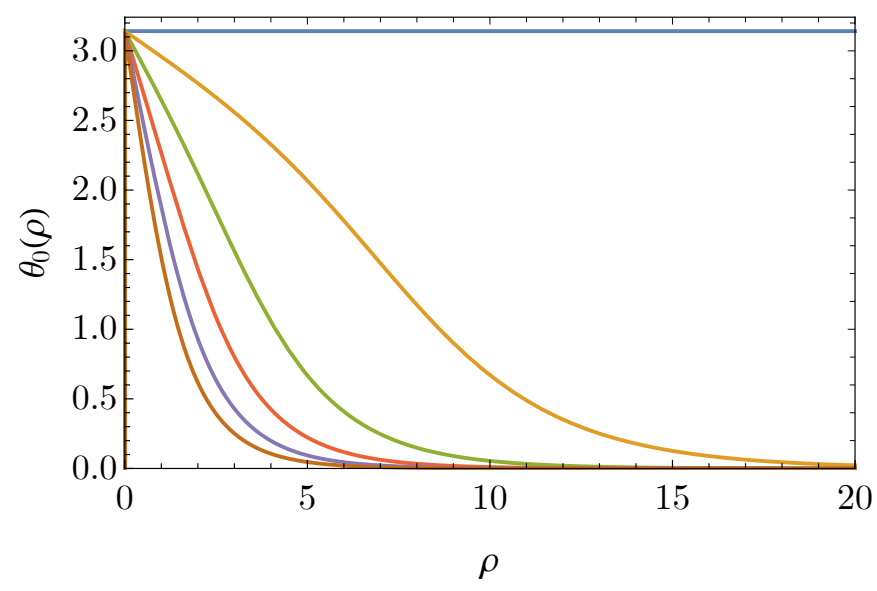

- $\mathrm{BJ}_{\mathrm{xy}} / D^{2}=0.0$

$-\mathrm{BJ}_{\mathrm{xy}} / D^{2}=0.2$

- $\mathrm{BJ}_{\mathrm{xy}} / D^{2}=0.4$

$-\mathrm{BJ}_{\mathrm{xy}} / D^{2}=0.6$

- $\mathrm{BJ}_{\mathrm{xy}} / D^{2}=0.8$

$-\mathrm{BJ}_{\mathrm{xy}} / D^{2}=1.0$

Figure 4.1: Configurations of the polar angle $\theta_{0}(\rho)$ of skyrmions for different values of $B J_{\mathrm{xy}} / D^{2}$.

or in Fourier space:

$-M \omega^{2}\left(\begin{array}{l}R_{\mathrm{A}} \\ R_{\mathrm{B}}\end{array}\right)=\left(\begin{array}{cc}-K+i \omega \beta_{\mathrm{eff}} \epsilon_{i j}-\alpha & \alpha \\ \alpha & -K+i \omega \beta_{\mathrm{eff}} \epsilon_{i j}-\alpha\end{array}\right)\left(\begin{array}{l}R_{\mathrm{A}} \\ R_{\mathrm{B}}\end{array}\right)$.

From these equations of motion, Eq. (4.17), we determine the eigenfrequencies as the eigenvalues of this equation. We express them as:

$$
\begin{aligned}
& \omega_{1}=\frac{\beta_{\mathrm{eff}}}{2 M} \pm \sqrt{\left(\frac{\beta_{\mathrm{eff}}}{2 M}\right)^{2}+\frac{K}{M}}, \\
& \omega_{2}=\frac{\beta_{\mathrm{eff}}}{2 M} \pm \sqrt{\left(\frac{\beta_{\mathrm{eff}}}{2 M}\right)^{2}+\frac{(2 \alpha+K)}{M}} .
\end{aligned}
$$

In the limit where the mass of the skyrmion goes to zero we can 


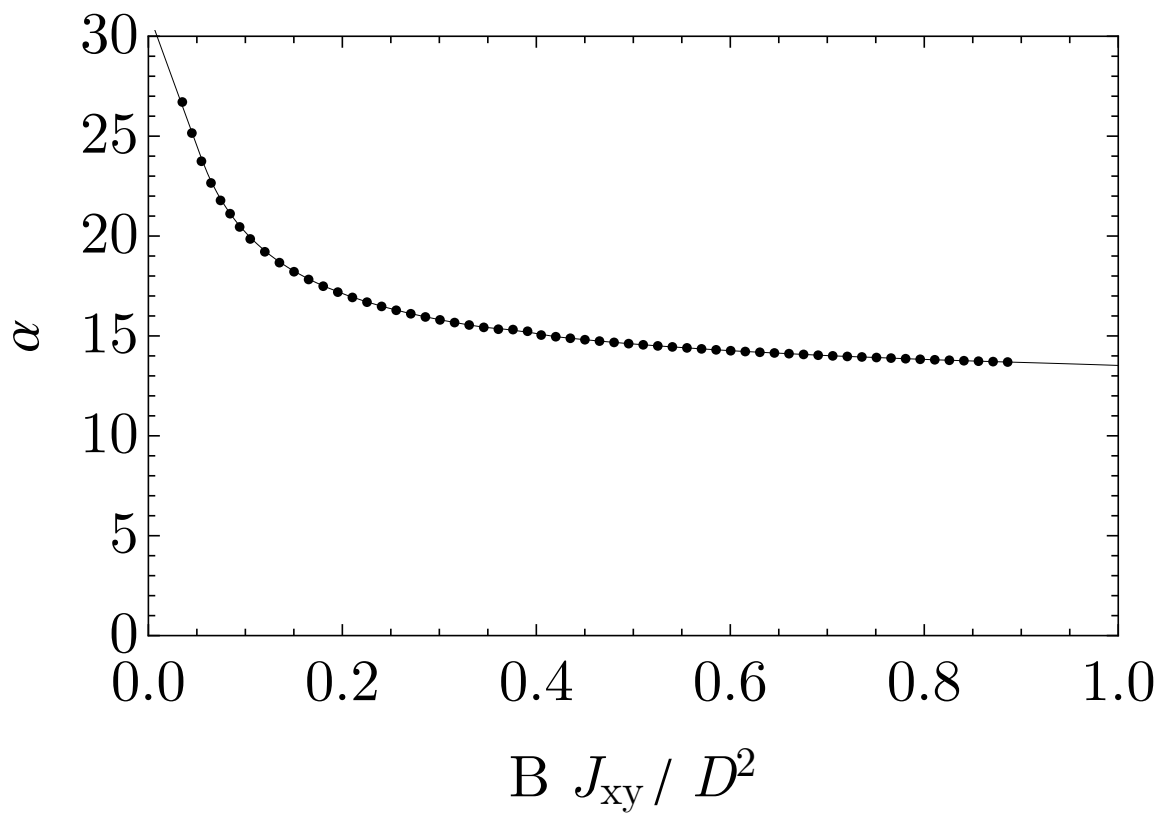

Figure 4.2: Coupling strength $\alpha$ between skyrmions in two layers for different magnitudes of external magnetic field $B J_{\mathrm{xy}} / D^{2}$. We chose $\rho_{\text {int }}=1$. The line is added to guide the eye. 
write the eigenfrequencies as:

$$
\begin{aligned}
& \omega_{1}= \pm \frac{K}{\beta_{\mathrm{eff}}}, \\
& \omega_{2}= \pm \frac{2 \alpha+K}{\beta_{\mathrm{eff}}} .
\end{aligned}
$$

Here we see that $\omega_{1}$ is independent of coupling $\alpha$ and we interpret that eigenmode as two skyrmions precessing in phase. On the other hand $\omega_{2}$ is dependent on the coupling $\alpha$ and we interpret this as the skyrmions precessing out of phase. Revisiting our eigenfrequencies including the mass term, Eq. (4.18) we see two differences. First, there is a split in energies between low and high frequencies where the massless term only has low frequencies. Second, the mass introduces a reduction of $\omega_{2}$ which is equivalent to the influence of mass in classical systems.

In figure Fig. 4.3 we plotted these eigenfrequencies for different values of magnetic field. Here you can see two constant eigenfrequencies, $\omega_{1}$ and two eigenfrequencies $\omega_{2}$ varying with the magnetic field. The influence of the coupling parameter $\alpha$ is clearly visible in $\omega_{2}$. We expect this aspect to be bigger for larger $J_{\mathrm{z}} / J_{\mathrm{xy}}$ and this is indeed visible.

\subsubsection{Antiferromagnetic regime}

In the antiferromagnetic regime we have to reassess the coupling parameter $\alpha$ since one can see from Eq. (4.11) that in this case $\alpha$ becomes negative. This means that for the antiferromagnetic region, our coupling has to be expanded to the fourth order. The fourth order term is also taken from Eq. (4.8) and will be referred to as $\gamma$ from now on. This leads to a effective potential:

$$
V_{0}=\frac{K}{2}\left(R_{\mathrm{T}}^{2}+R_{\mathrm{B}}^{2}\right)-\frac{\alpha}{2}\left(R_{\mathrm{T}}-R_{\mathrm{B}}\right)^{2}+\frac{\gamma}{4}\left(R_{\mathrm{T}}-R_{\mathrm{B}}\right)^{4} .
$$




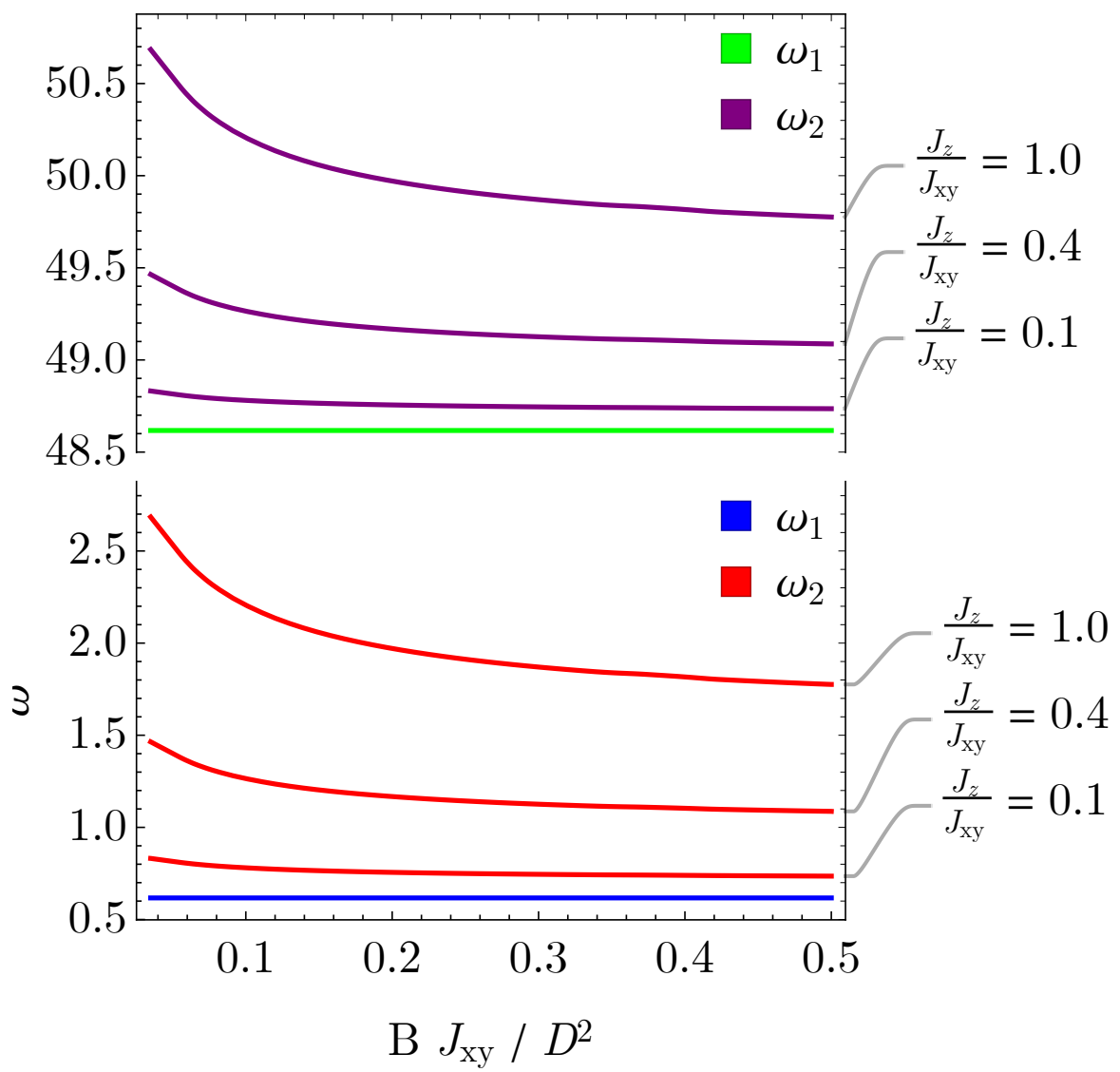

Figure 4.3: Eigenfrequencies of two ferromagnetically coupled layers with a skyrmion plotted versus magnetic field $B J_{\mathrm{xy}} / D^{2}$. Here we plotted for different coupling strengths $J_{\mathrm{z}} / J_{\mathrm{xy}}=0.1,0.4$ and 1.0 , and chose $M=0.5, \beta_{\text {eff }}=24, K=15$. The colors indicate the four different ferromagnetic eigenfrequencies. 
Using coordinate transformation Eq. (4.9) and thereafter varying this with respect to $R$, which gives: $R=0$, we end up with the potential:

$$
V_{0}=K \mathbf{r}^{2}-\frac{\alpha}{2}(2 \mathbf{r})^{2}+\frac{\gamma}{4}(2 \mathbf{r})^{4}
$$

The minimum of this potential is given by:

$$
r_{0}= \pm \sqrt{\frac{2 \alpha-K}{8 \gamma}},
$$

if $2 \alpha>K$. If $2 \alpha<K$ we take the minimum $r_{0}=0$. Thus, for antiferromagnetical coupling we have to calculate the eigenfrequencies for both cases.

The first case: $2 \alpha>K$. We vary our effective action Eq. (4.12) including the new effective potential Eq. (4.20) around $r_{0}$, Eq. (4.22). Solving these equations of motion leads to the following eigenfrequencies:

$$
\begin{aligned}
& \omega_{1}=\frac{\beta_{\mathrm{eff}}}{2 M} \pm \sqrt{\left(\frac{\beta_{\mathrm{eff}}}{2 M}\right)^{2}+\frac{K}{M}}, \\
& \omega_{2}=\frac{\beta_{\mathrm{eff}}}{2 M} \pm \sqrt{\left(\frac{\beta_{\mathrm{eff}}}{2 M}\right)^{2}+\frac{(2 \alpha-K)}{M}} .
\end{aligned}
$$

And for the case $2 \alpha<K$, we perturb the same action around $r_{0}=0$. This leads to the eigenfrequencies:

$$
\begin{aligned}
& \omega_{1}=\frac{\beta_{\mathrm{eff}}}{2 M} \pm \sqrt{\left(\frac{\beta_{\mathrm{eff}}}{2 M}\right)^{2}+\frac{K}{M}}, \\
& \omega_{2}=\frac{\beta_{\mathrm{eff}}}{2 M} \pm \sqrt{\left(\frac{\beta_{\mathrm{eff}}}{2 M}\right)^{2}+\frac{(K-2 \alpha)}{M}} .
\end{aligned}
$$



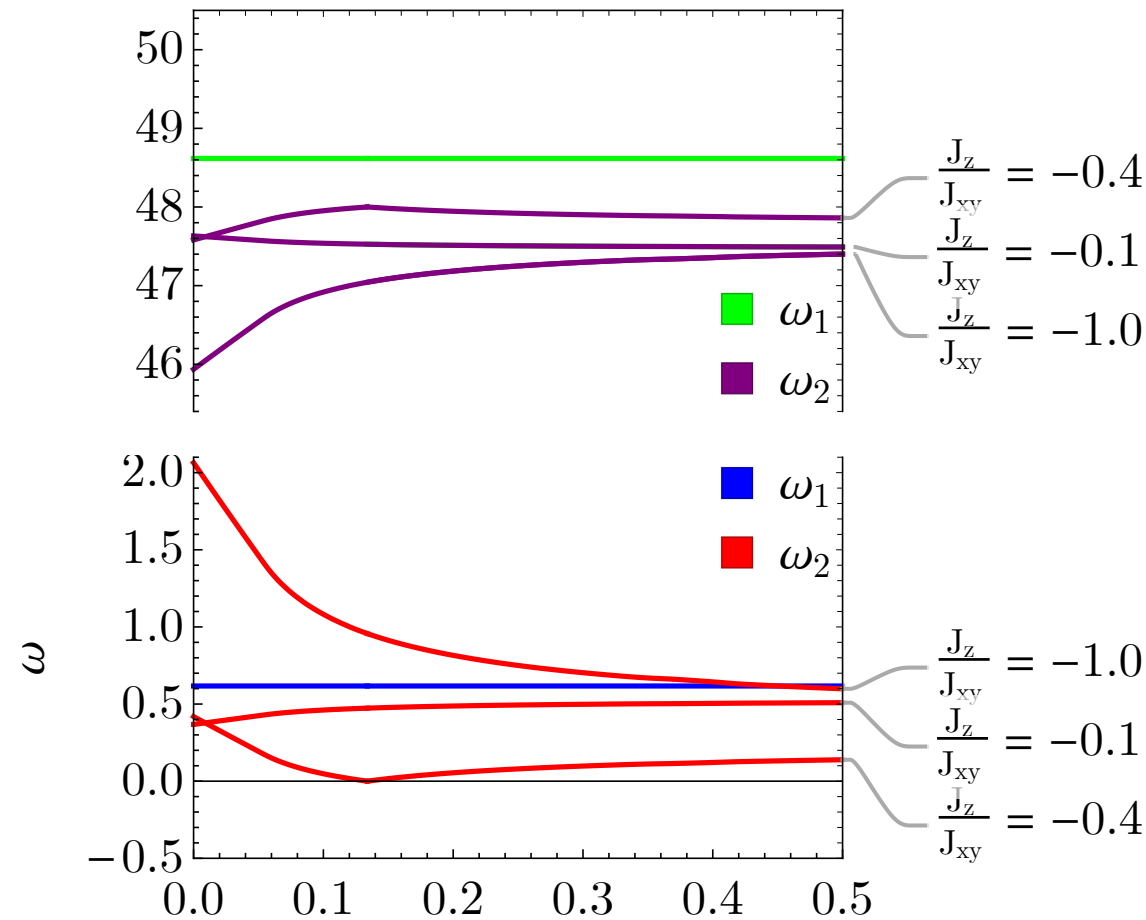

B $J_{\mathrm{xy}} / D^{2}$

Figure 4.4: Eigenfrequencies of two antiferromagnetic coupled layers with a skyrmion plotted versus magnetic field $B J_{\mathrm{xy}} / D^{2}$. Here we plotted for different coupling strengths $J_{\mathrm{z}} / J_{\mathrm{xy}}=-0.1,-0.4$ and -1.0 , and chose $M=0.5, \beta_{\mathrm{eff}}=24, K=15$. The colors indicate the four different ferromagnetic eigenfrequencies. The kink in the frequencies occur at $K=2 \alpha$ where the border is between the two cases of our potential minimum $r_{0}$. 
In Fig. 4.4 the eigenfrequencies for antiferromagnetic coupling show comparable behavior to the ferromagnetic ones. Two eigenfrequencies independent from magnetic field which are around the same frequencies as the ferromagnetic frequencies, and two eigenfrequencies dependent on the interlayer coupling. The transition point at $2 \alpha=K$ clearly leaves it marks. The line of $J_{z} / J_{\mathrm{xy}}=0.4$ is discontinuous at $\omega_{2}=0$ which is where this transition point is. Furthermore, the frequencies for small coupling, $J_{z} / J_{x y}=0.1$, and large coupling, $J_{\mathrm{z}} / J_{\mathrm{xy}}=0.4$, have opposite reactions to the magnetic field since they are both positioned at different sides of the transition point.

\subsection{Numerical method}

The eigenfrequencies of the confined skyrmion can also be obtained using numerical simulations. Here we simulate a system containing skyrmions using Monte Carlo techniques. These Monte Carlo simulations only give stationary solutions since we are looking at averages. We will include fluctuations in the stationary states to determine the dynamics of such systems. Below we will describe the Monte Carlo simulations and the dynamical solutions for these configurations and present their results.

\subsubsection{Monte Carlo}

As previous described in chapters Chapter 2 and Chapter 3 we use Monte Carlo systems to simulate magnetic systems containing skyrmions. We used simulated annealing where we start from a random spin configuration at a high temperature and slowly lowering the temperature until a configuration close to the ground state is found. Here it is important that the configuration resembles the ground state since the following calculations only work for the ground state or a state really close to it. In this paper we are interested in single skyrmion behavior, i.e. we only want 
one skyrmion in each layer. To construct such a state we included open boundary conditions. This forces the skyrmion to sit in the middle of the layer. This also increases the phase space where only one skyrmion in stead of a lattice of skyrmions is found. We simulated for various parameters of magnetic field and interlayer coupling, for simplicity we chose to not include anisotropy. For these parameters, four phases were found: the polarized phase, where the ferromagnetic intralayer coupling and external magnetic field are dominant and all spins point in the same direction. The spiral phase where the DMI is dominant and spirals are formed. And two skyrmion phases where we found skyrmions and identified them by a non-zero winding number. The difference between the two phases is that one phase has a singular skyrmion per layer and the other a lattice of multiple skyrmions. In this paper we are only interested in systems with only one skyrmion and thus a winding number of \pm 1 in a layer. We found skyrmions in the ferromagnetic as well the antiferromagnetic interlayer coupling region. Because of their repellent nature the parameter space is smaller for the antiferromagnetic coupling, since there is less space to fit two skyrmions inside the boundaries. In Fig. 4.5 we show one of the skyrmion configurations. This is an example of one of the systems used in the analysis below.

\subsubsection{Non-interacting magnons}

In this section we describe the excited states of the systems found using the Monte Carlo simulations. We calculate the excited energies as follows. We start with the Hamiltonian Eq. (4.3) and view the spins quantum mechanically. To do calculations with these small spins we need to preform the Holstein Primakoff transformation to transform the spin operators $S_{i}$ to bosonic operators $a, a^{\dagger}$ 


\section{FM coupling \\ Layer 1}

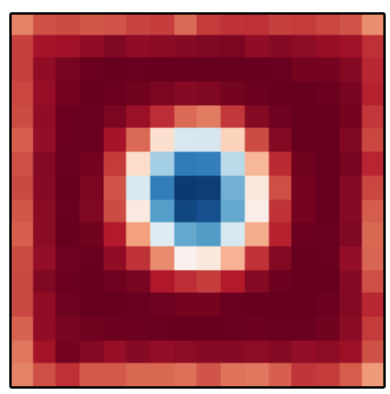

Layer 2

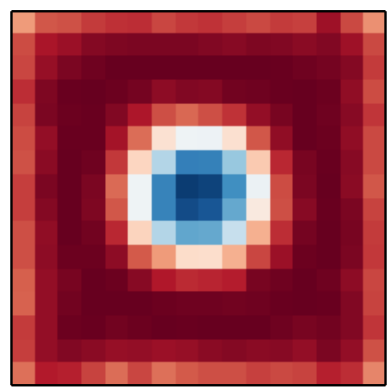

AFM coupling

Layer 1

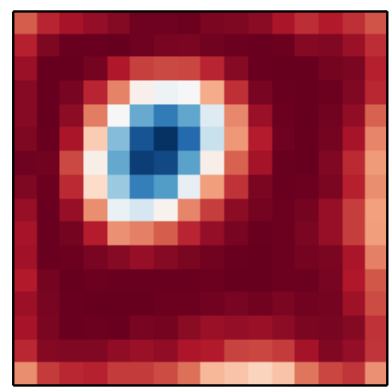

Layer 2

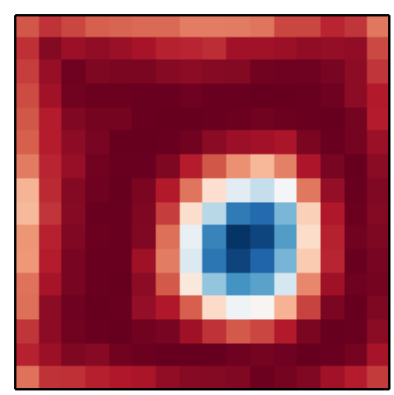

1.0

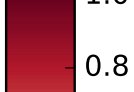

0.6

0.4

0.2

0.0

$-0.2$

$-0.4$

$-0.6$

$-0.8$

Figure 4.5: Configurations of bilayer skyrmion systems with open boundaries for ferromagnetic coupling (FM) and antiferromagnetic coupling (AFM). Here the z-component of the spin system is plotted for both layers 1 and 2. The repellent character of the open boundaries is clearly visible in both cases since the skyrmions are placed in the center of the layers, furthermore the AFM case shows also some repulsion between both skyrmions themselves. Both skyrmions have the same pitch $D / J_{\mathrm{xy}}=\arctan (2 \pi / 10)$, and anisotropy $K J_{\mathrm{xy}} / D^{2}=0$. For FM the interlayer coupling strength is $J_{\mathrm{z}} / J_{\mathrm{xy}}=0.11$ and $B J_{\mathrm{xy}} / D^{2}=0.56$. In the AFM case $J_{\mathrm{z}} / J_{\mathrm{xy}}=-0.11$ and $B J_{\mathrm{xy}} / D^{2}=0.69$. 
through:

$$
\begin{aligned}
& s_{+}=\hbar \sqrt{2 s} \sqrt{1-\frac{a^{\dagger} a}{2 s} a}, \\
& s_{-}=\hbar \sqrt{2 s} a^{\dagger} \sqrt{1-\frac{a^{\dagger} a}{2 s}}, \\
& s_{z}=\hbar\left(s-a^{\dagger} a\right) .
\end{aligned}
$$

We expand the square root to second order. The first order gives us the classical energy of the system $E_{\mathrm{cl}}$ and the second order gives us the non-interacting magnons.

$$
H=E_{\mathrm{cl}}+E_{0}+\left(\mathbf{a}^{\dagger}, \mathbf{a}\right) \mathbf{H}\left(\begin{array}{c}
\mathbf{a}^{\dagger} \\
\mathbf{a}
\end{array}\right),
$$

where the matrix $\mathbf{H}$ and vector $\left(\mathbf{a}^{\dagger} \mathbf{a}\right)$ has components labeled by lattice positions. This gives a non diagonal hamiltonian $\mathbf{H}$. To diagonalize this $\mathbf{H}$, we use the Bogoliubov approach. This leads to a diagonal matrix for in terms of new operators $\left(\gamma, \gamma^{\dagger}\right)$. The subsequent hamiltonian looks like:

$$
H=E_{\mathrm{cl}}+E_{0}+\sum_{i=1}^{N} \hbar \omega_{i}\left(\gamma_{i}^{\dagger} \gamma_{i}+\frac{1}{2}\right) .
$$

Where $\left(\begin{array}{c}\gamma_{i}^{\dagger} \\ \gamma_{i}\end{array}\right)=\boldsymbol{\Gamma}\left(\begin{array}{c}\mathbf{a}^{\dagger} \\ \mathbf{a}\end{array}\right)$ and $i$ labels the modes. The ground state energy is thus:

$$
E_{\mathrm{GS}}=E_{\mathrm{cl}}+E_{0}+\frac{1}{2} \sum_{i=1}^{N} \hbar \omega_{i} .
$$

This shift in the energy of ground state state caused by quantum fluctuations is known the as zero-point energy [112]:

$$
E_{\mathrm{ZP}} \equiv E_{\mathrm{GS}}-E_{\mathrm{cl}}=E_{0}+\frac{1}{2} \sum_{i=1}^{N} \hbar \omega_{i} \text {. }
$$


The collective mode frequency can be calculated from the ground state of our system. We define the ground state as:

$$
\gamma_{i}|\mathrm{GS}\rangle=0
$$

from where we can calculate the number of magnons per lattice site as

$$
\rho_{i}^{0}=\left\langle\mathrm{GS}\left|a_{i}^{\dagger} a_{i}\right| \mathrm{GS}\right\rangle=\sum_{p}\left|v_{i p}\right|^{2}
$$

In the same manner we can describe the extra energy which spins obtain from exciting the system with magnons. We define the excited state:

$$
\left|\psi_{k}\right\rangle=\gamma_{k}^{\dagger}|\mathrm{GS}\rangle
$$

for magnon mode $k$. This gives a number of magnons at site $i$ as

$$
\rho_{i, k}^{1}=\left\langle\psi_{k}\left|a_{i}^{\dagger} a_{i}\right| \psi_{k}\right\rangle=\sum_{p}\left|v_{i p}\right|^{2}+\left|u_{i k}\right|^{2}+\left|v_{i k}\right|^{2} .
$$

Finally we can compute the added energy per spin by computing the difference between the zero point and the excited mode energy. Furthermore, an indication of the nature of the mode is given by its magnon number relative to the ground state:

$$
\Delta \rho_{i, k}=\rho_{i, k}^{1}-\rho_{i}^{0}=\left|u_{i k}\right|^{2}+\left|v_{i k}\right|^{2} .
$$

In Fig. 4.6 we plotted the distribution of the magnon modes on a skyrmion system from our Monte Carlo simulations. Here we show the first two modes which are the well studied precession (a) and breathing (b) mode, and an excited mode (c).

Using this framework we calculated the energies of skyrmion systems for a range of interlayer couplings. In Fig. 4.7 we show the energies of the three first excited modes, here we see that the precession and breathing mode have no dependence on the interlayer 

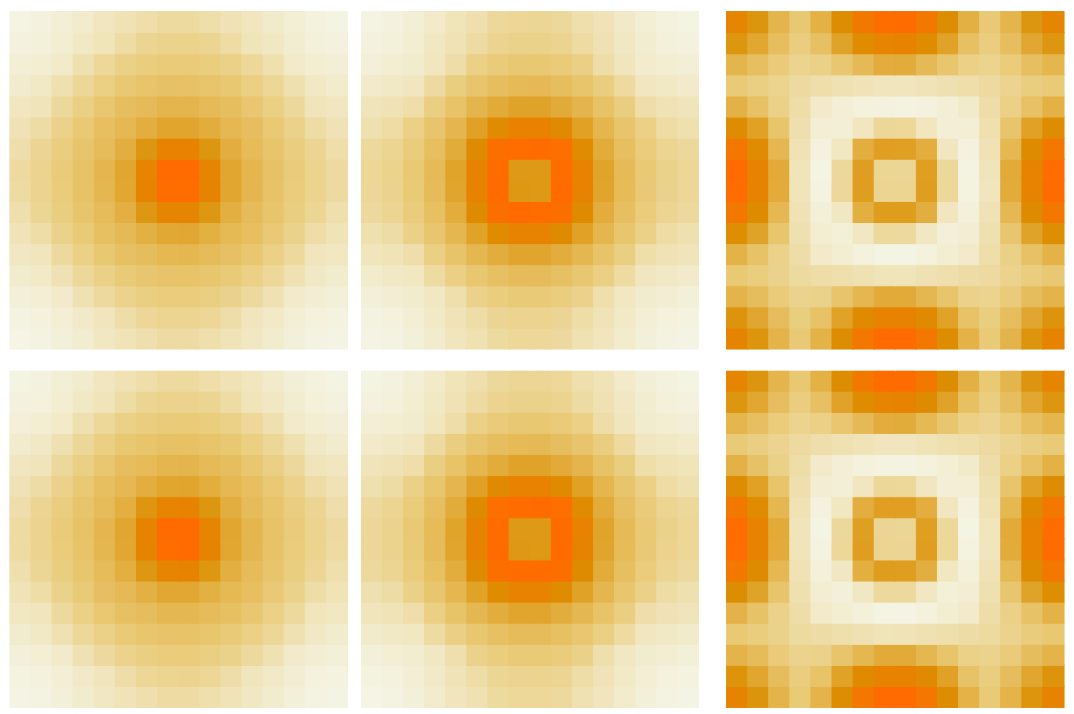

Figure 4.6: Magnon activity $\Delta \rho_{i, k}$ for a $16 \times 16$ bilayer system with a skyrmion with pitch length $p=10$. (a) is the lowest "precession" state, b) the first excited "breathing" state and c) the an excited state. 


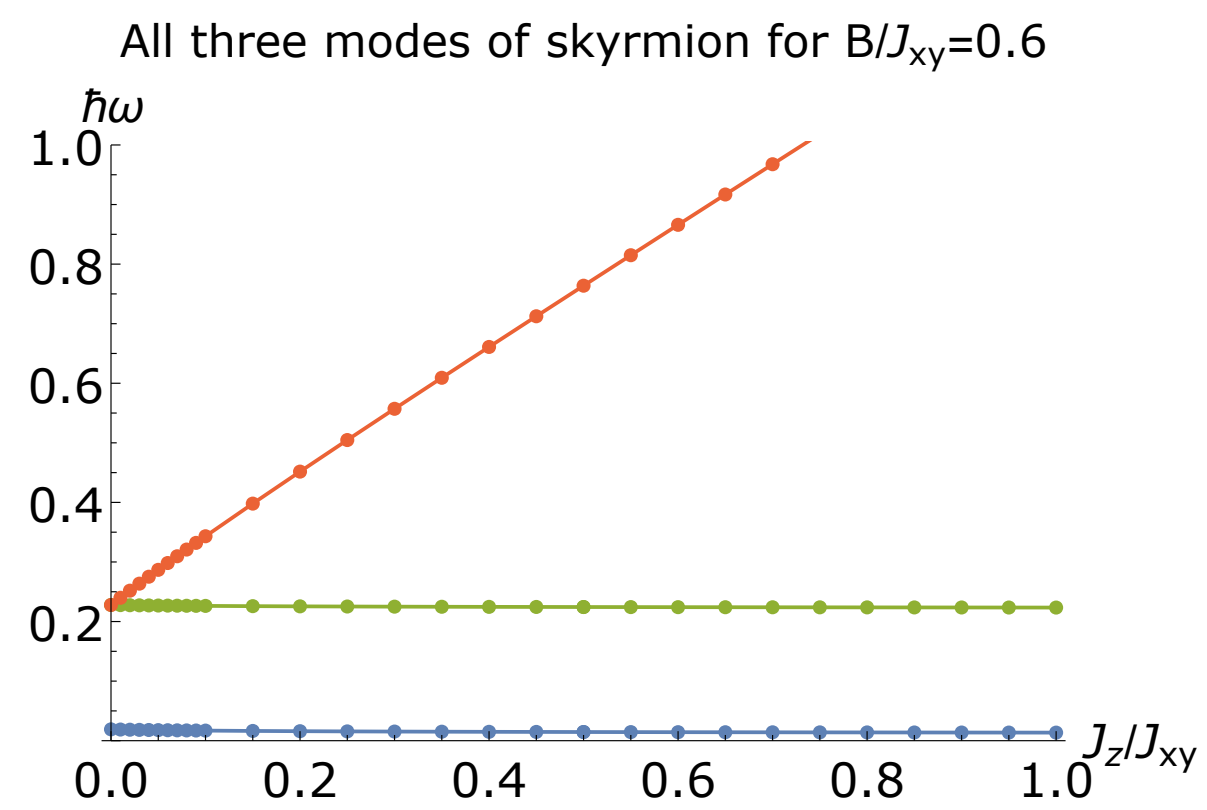

Figure 4.7: Energies of the three lowest magnon modes of a skyrmion state with pitch $p=8$, and $B J_{\mathrm{xy}} / D=0.6$ for different values of $J_{\mathrm{z}} / J_{\mathrm{xy}}$.

coupling. Contrarily, the next excited state has a linear dependency on the interlayer coupling $J_{\mathrm{z}} / J_{\mathrm{xy}}$. We preformed comparable calculations for a range of magnetic field parameters which we show in Fig. 4.8. A clear influence of the magnetic field is visible. This leads to a large energy difference for the higher excited state and small differences for the precession and breathing mode.

\subsection{Discussion}

Both analytical and numerical results are found for spin waves in systems containing skyrmions. Comparing these numerical results with the previous analytical results a few things stand out. First, 
All three modes of skyrmion

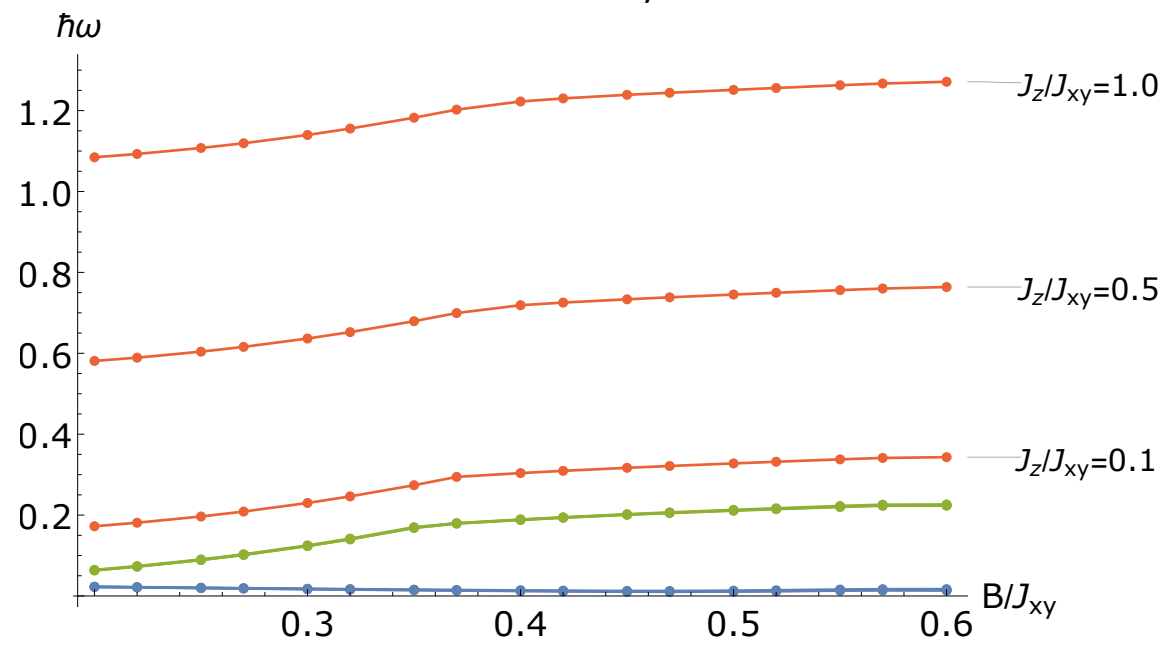

Figure 4.8: Energies of the three lowest magnon modes of a skyrmion state with pitch $p=8$ different values of $J_{z} / J_{\mathrm{xy}}$ and various values of magnetic field $B J_{\mathrm{xy}} / D$. Also shown: b) detailed version of the breathing mode and c) precession mode for different values of $J_{\mathrm{z}} / J_{\mathrm{xy}}$ 
the overall structure is comparable with a flat response to magnetic field for one of the frequencies ( $\omega_{1}$ and the lowest precession state). And second, the higher frequencies are proportional dependent on the interlayer coupling. A difference is that the numerical method delivers more states and shows a breathing mode which does not exhibit a strong dependence on the interlayer coupling.

Unfortunately, we could not compare the two methods in the antiferromagnetic interlayer coupling region since we could not find a positive definite Hamiltonian describing the physics in the antiferromagnetic interlayer coupling domain numerically.

We are hopeful that our results show enough fingerprints to detect two coupled skyrmion layers by finding a distinct combination of constant and rising energies as a function of the coupling. Furthermore, we show how the different procession modes look like in a bilayer system which can be used to identify skyrmions as well. The antiferromagnetic region is interesting to further investigate, the different behaviors above and below the critical point $2 \alpha=K$ make them very recognizable and suitable for future applications. 


\section{Outlook}

In this thesis we focussed on chiral effects in magnetic multilayers. Those multilayers already proved to posses qualities which are favorable for new applications such as making skyrmions more stable and nullifying the Magnus effect which is a big hurdle for applications with moving skyrmions. We added new insight to the family of effects originating from multilayer systems. In Chapter 2, we showed that skyrmion lattices in two layers can change their configuration. The natural lattice configuration for skyrmions is hexagonal, but under influence of antiferromagnetic coupling between two layers this configuration can be changed to a square one. The nature of this transition is such that we can adjust the lattice configuration with external forces. This is promising for new applications, e.g. the number of skyrmions a spin wave meets when traveling through a system can be controlled by switching skyrmion lattice configurations. In Chapter 3 we showed that helical spin spirals are also influenced by the added complexity of stacking layers. In this case we showed that coupled layers with alternating DMI have an extensive phase space. Using the interlayer coupling, the turning sense and wavelength of the spirals can be tuned. Interesting to note is that number of layers has a big influence on this effect: Increasing the number of layers showed a stronger effect on the wavelength of the spirals. Also, an even odd effect was found, where we showed that in systems with an even number of layers the spirals can disappear completely for a strong 
interaction between the layers. This gives rise for new applications as well since the phase transition between the spiral directions and the transition turning the spirals on and off are controllable externally. In the final chapter Chapter 4 we showed how to detect skyrmions in multilayers using microwave ferromagnetic resonance spectroscopy. This is important because when multiple layers are stacked, most techniques only probe the top layer. In our research we show the distinct resonance of two coupled skyrmions.

As discussed above, the added complexity by adding layers is very promising for new creative applications. More research is needed to fully reap the promise of multilayers in chiral systems. The Monte Carlo simulations we performed were limited and can be expanded upon by using more sophisticated algorithms. Furthermore, the research of Chapter 2 and Chapter 3 can be combined: When adding more layers in Chapter 2 this will possibly give opportunities to construct more skyrmion lattice configurations. Moreover, using different values of DMI in both layers more complex skyrmion lattices can be constructed such as an honeycomb skyrmion lattice. The work of Chapter 3 can be expanded by including anisotropy and an external magnetic field to create skyrmions with different winding numbers. It will be interesting to study how these skyrmions will form and what lattice structure they will adopt depending on the number of layers. This can possibly lead to other interesting skyrmion lattices. Finally, it is possible to tie this whole thesis together by calculating the microwave ferromagnetic resonance of the systems described in Chapter 2 and 3 , and those mentioned above. This will guide experimental research with detecting exotic skyrmion lattice configurations and spiral wave structures in the future.

While experimental verification of our results are, as of writing, not realized, the systems described in this thesis are well within the current technical abilities and hopefully are of interest for experimental groups. 


\section{Bibliography}

${ }^{1}$ J. M. Coey, Magnetism and magnetic materials, Vol. 9780521816144 (2010).

${ }^{2}$ W. Gilbert, De Magnete (1600).

${ }^{3}$ J. C. Maxwell, "VIII. A dynamical theory of the electromagnetic field", Philosophical Transactions of the Royal Society of London 155, 10.1098/rstl.1865.0008 (1865).

${ }^{4}$ A. Aharoni and A. Arrott, "Introduction to the Theory of Ferromagnetism", Physics Today 50, 10.1063/1.881916 (1997).

${ }^{5} \mathrm{P}$. Weiss, "La variation du ferromagnétisme avec la température", Comptes Rendus 143, 1136-1139 (1906).

${ }^{6} \mathrm{~N}$. Bohr, Studier over metallernes elektrontheori (Thaning \& Appel in Komm., 1911).

${ }^{7}$ H. J. Van Leeuwen, Vraagstukken uit de electronentheorie van het magnetisme (Eduard IJdo, 1919).

${ }^{8}$ P. A. M. Dirac, "Quantum mechanics of many-electron systems", Proceedings of the Royal Society of London. Series A, Containing Papers of a Mathematical and Physical Character 123, 714733 (1929).

${ }^{9}$ L. Néel, "Propriétés magnétiques de l'état métallique et énergie d'interaction entre atomes magnétiques", Annales de Physique 11, 232-279 (1936). 
${ }^{10}$ C. G. Shull, W. A. Strauser, and E. O. Wollan, "Neutron diffraction by paramagnetic and antiferromagnetic substances", Physical Review 83, 333-345 (1951).

${ }^{11}$ E. Fawcett, "Spin-density-wave antiferromagnetism in chromium", Rev. Mod. Phys. 60, 209-283 (1988).

${ }^{12}$ W. L. Roth, "Magnetic structures of mno, feo, coo, and nio", Phys. Rev. 110, 1333-1341 (1958).

${ }^{13}$ T. Jungwirth, X Marti, P Wadley, and J Wunderlich, "Antiferromagnetic spintronics", Nature nanotechnology 11, 231-241 (2016).

${ }^{14}$ J. C. Slonczewski, "Conductance and exchange coupling of two ferromagnets separated by a tunneling barrier", Physical Review B 39, 6995-7002 (1989).

${ }^{15}$ S. S. P. Parkin, N. More, and K. P. Roche, "Oscillations in exchange coupling and magnetoresistance in metallic superlattice structures: $\mathrm{Co} / \mathrm{Ru}, \mathrm{Co} / \mathrm{Cr}$, and Fe/Cr", Physical Review Letters 64, 2304-2307 (1990).

${ }^{16}$ D. M. Edwards, J. Mathon, R. B. Muniz, and M. S. Phan, "Oscillations of the exchange in magnetic multilayers as an analog of de Haas van Alphen effect", Physical Review Letters 67, 493496 (1991).

${ }^{17}$ P. Bruno, "Theory of interlayer magnetic coupling", Physical Review B 52, 411-439 (1995).

${ }^{18}$ J. Faure-Vincent, C. Tiusan, C. Bellouard, E. Popova, M. Hehn, F. Montaigne, and A. Schuhl, "Interlayer Magnetic Coupling Interactions of Two Ferromagnetic Layers by Spin Polarized Tunneling", Physical Review Letters 89, 107206 (2002). 
${ }^{19}$ I. Troyanchuk, M. Bushinsky, V Sikolenko, V Efimov, C Ritter, T Hansen, and D. Többens, "Pressure induced antiferromagnetferromagnet transition in la 0.5 ba 0.5 coo 2.8 cobaltite", The European Physical Journal B 86, 435 (2013).

${ }^{20}$ T. Song, Z. Fei, M. Yankowitz, Z. Lin, Q. Jiang, K. Hwangbo, Q. Zhang, B. Sun, T. Taniguchi, K. Watanabe, M. A. McGuire, D. Graf, T. Cao, J. H. Chu, D. H. Cobden, C. R. Dean, D. Xiao, and $\mathrm{X}$. Xu, Switching $2 \mathrm{D}$ magnetic states via pressure tuning of layer stacking, 2019.

${ }^{21}$ M. Fechner, P. Zahn, S. Ostanin, M. Bibes, and I. Mertig, "Switching Magnetization by 180 with an Electric Field", Physical Review Letters 108, 197206 (2012).

${ }^{22}$ User:Hankwang, "Https://commons.wikimedia.org/wiki/file: hard_drive_capacity_over_time.svg", [Online; accessed 25June-2020] (2018).

${ }^{23}$ J. W. Nielsen, Magnetic Bubble Materials, tech. rep. (1979).

${ }^{24}$ A. Eschenfelder and A. H. Bobeck, "Magnetic bubble technology", PhT 33, 55 (1980).

${ }^{25}$ B. Dieny, V. S. Speriosu, S. S. Parkin, B. A. Gurney, D. R. Wilhoit, and D. Mauri, "Giant magnetoresistive in soft ferromagnetic multilayers", Physical Review B 43, 1297-1300 (1991).

${ }^{26}$ M. N. Baibich, J. M. Broto, A. Fert, F. N. Van Dau, F. Petroff, P. Eitenne, G. Creuzet, A. Friederich, and J. Chazelas, "Giant magnetoresistance of (001)Fe/(001)Cr magnetic superlattices", Physical Review Letters 61, 2472-2475 (1988).

${ }^{27}$ G. Binasch, P. Grünberg, F. Saurenbach, and W. Zinn, "Enhanced magnetoresistance in layered magnetic structures with antiferromagnetic interlayer exchange", Physical Review B 39, 4828-4830 (1989). 
${ }^{28}$ R. Schad, C. D. Potter, P. Beliën, G. Verbanck, V. V. Moshchalkov, and Y. Bruynseraede, "Giant magnetoresistance in $\mathrm{Fe} / \mathrm{Cr}$ superlattices with very thin Fe layers", Applied Physics Letters 64, 3500-3502 (1994).

${ }^{29}$ A. Fert, "Nobel lecture: origin, development, and future of spintronics", Reviews of modern physics 80, 1517 (2008).

${ }^{30}$ S. S. P. Parkin, M. Hayashi, and L. Thomas, "Magnetic domainwall racetrack memory.", Science (New York, N.Y.) 320, 190-4 (2008).

${ }^{31} \mathrm{~S}$. Mittal, "A survey of techniques for architecting processor components using domain-wall memory", ACM Journal on Emerging Technologies in Computing Systems (JETC) 13, 1-25 (2016).

${ }^{32}$ K.-J. Kim, S. K. Kim, Y. Hirata, S.-H. Oh, T. Tono, D.-H. Kim, T. Okuno, W. S. Ham, S. Kim, G. Go, Y. Tserkovnyak, A. Tsukamoto, T. Moriyama, K.-J. Lee, and T. Ono, "Fast domain wall motion in the vicinity of the angular momentum compensation temperature of ferrimagnets", Nature Materials 16, 11871192 (2017).

${ }^{33}$ A. Fert, V. Cros, and J. Sampaio, "Skyrmions on the track", Nature Nanotechnology 2013 8:3 (2013).

${ }^{34}$ I. Dzyaloshinsky, "A thermodynamic theory of weak ferromagnetism of antiferromagnetics", Journal of Physics and Chemistry of Solids 4, 241-255 (1958).

${ }^{35}$ T. Moriya, "Anisotropic Superexchange Interaction and Weak Ferromagnetism", Physical Review 120, 91-98 (1960).

${ }^{36}$ A. Fert and P. M. Levy, "Role of anisotropic exchange interactions in determining the properties of spin-glasses", Physical Review Letters 44, 1538-1541 (1980). 
${ }^{37} \mathrm{~A}$. Bogdanov and A. Hubert, "Thermodynamically stable magnetic vortex states in magnetic crystals", Journal of Magnetism and Magnetic Materials 138, 255-269 (1994).

${ }^{38} \mathrm{~T}$. Skyrme, "A unified field theory of mesons and baryons", Nuclear Physics 31, 556-569 (1962).

${ }^{39}$ I. Zahed and G. E. Brown, The Skyrme model, 1986.

${ }^{40}$ S. Muhlbauer, B. Binz, F. Jonietz, C. Pfleiderer, A. Rosch, A. Neubauer, R. Georgii, and P. Boni, "Skyrmion Lattice in a Chiral Magnet", Science 323, 915-919 (2009).

${ }^{41}$ X. Z. Yu, Y. Onose, N. Kanazawa, J. H. Park, J. H. Han, Y. Matsui, N. Nagaosa, and Y. Tokura, "Real-space observation of a two-dimensional skyrmion crystal", Nature 465, 901-904 (2010).

${ }^{42}$ S. Heinze, K. von Bergmann, M. Menzel, J. Brede, A. Kubetzka, R. Wiesendanger, G. Bihlmayer, and S. Blügel, "Spontaneous atomic-scale magnetic skyrmion lattice in two dimensions", Nature Physics 7, 713-718 (2011).

${ }^{43}$ W. Münzer, A. Neubauer, T. Adams, S. Mühlbauer, C. Franz, F. Jonietz, R. Georgii, P. Böni, B. Pedersen, M. Schmidt, A. Rosch, and C. Pfleiderer, "Skyrmion lattice in the doped semiconductor Fe1-xCo xSi", Physical Review B - Condensed Matter and Materials Physics 81, 041203 (2010).

${ }^{44}$ X. Z. Yu, N. Kanazawa, Y. Onose, K. Kimoto, W. Z. Zhang, S. Ishiwata, Y. Matsui, and Y. Tokura, "Near room-temperature formation of a skyrmion crystal in thin-films of the helimagnet FeGe", Nature Materials 10, 106-109 (2011).

${ }^{45}$ S. Woo, K. Litzius, B. Krüger, M.-Y. Im, L. Caretta, K. Richter, M. Mann, A. Krone, R. M. Reeve, M. Weigand, P. Agrawal, I. Lemesh, M.-A. Mawass, P. Fischer, M. Kläui, and G. S. D. Beach, "Observation of room-temperature magnetic skyrmions 
and their current-driven dynamics in ultrathin metallic ferromagnets", Nature Materials 15, 501-506 (2016).

${ }^{46}$ T. Okubo, S. Chung, and H. Kawamura, "Multiple-q states and the Skyrmion lattice of the triangular-lattice Heisenberg antiferromagnet under magnetic fields", Physical Review Letters 108, 017206 (2012).

${ }^{47}$ M. Azhar and M. Mostovoy, "Incommensurate Spiral Order from Double-Exchange Interactions", Physical Review Letters 118, 027203 (2017).

${ }^{48}$ S. L. Sondhi, A. Karlhede, S. A. Kivelson, and E. H. Rezayi, "Skyrmions and the crossover from the integer to fractional quantum Hall effect at small Zeeman energies", Physical Review B 47, 16419-16426 (1993).

${ }^{49}$ S. E. Barrett, G. Dabbagh, L. N. Pfeiffer, K. W. West, and R. Tycko, "Optically pumped NMR evidence for finite-size Skyrmions in GaAs quantum wells near Landau level filling $\nu=1 "$, Physical Review Letters 74, 5112-5115 (1995).

${ }^{50} \mathrm{P}$. Sutcliffe, Chiral ferromagnetic fluids: Let's twist again, 2017.

${ }^{51} \mathrm{P}$. Corboz, "Advanced numerical methods in many-body physics", (2015).

${ }^{52} \mathrm{~N}$. Metropolis, A. W. Rosenbluth, M. N. Rosenbluth, A. H. Teller, and E. Teller, "Equation of state calculations by fast computing machines", The Journal of Chemical Physics 21, 10871092 (1953).

${ }^{53}$ W. K. Hastings, "Monte Carlo sampling methods using Markov chains and their applications", Biometrika 57, 97-109 (1970).

${ }^{54}$ E. Van Walsem, R. A. Duine, J. Lucassen, R. Lavrijsen, and H. J. Swagten, "Structural transitions of skyrmion lattices in synthetic antiferromagnets", Physical Review B 100, 064402 (2019). 
${ }^{55}$ S. Muhlbauer, B. Binz, F. Jonietz, C. Pfleiderer, A. Rosch, A. Neubauer, R. Georgii, and P. Boni, "Skyrmion Lattice in a Chiral Magnet", Science 323, 915-919 (2009).

${ }^{56}$ B. Dupé, M. Hoffmann, C. Paillard, and S. Heinze, "Tailoring magnetic skyrmions in ultra-thin transition metal films", Nature Communications 5, 4030 (2014).

${ }^{57}$ G. Yu, P. Upadhyaya, X. Li, W. Li, S. K. Kim, Y. Fan, K. L. Wong, Y. Tserkovnyak, P. K. Amiri, and K. L. Wang, "RoomTemperature Creation and Spin Orbit Torque Manipulation of Skyrmions in Thin Films with Engineered Asymmetry", Nano Letters 16, 1981-1988 (2016).

${ }^{58}$ A. Soumyanarayanan, M. Raju, A. L. Gonzalez Oyarce, A. K. C. Tan, M.-Y. Im, A. Petrović, P. Ho, K. H. Khoo, M. Tran, C. K. Gan, F. Ernult, and C. Panagopoulos, "Tunable roomtemperature magnetic skyrmions in $\mathrm{Ir} / \mathrm{Fe} / \mathrm{Co} / \mathrm{Pt}$ multilayers", Nature Materials 16, 898-904 (2017).

${ }^{59}$ C. Moreau-Luchaire, C. Moutafis, N. Reyren, J. Sampaio, C. A. F. Vaz, N. Van Horne, K. Bouzehouane, K. Garcia, C. Deranlot, P. Warnicke, P. Wohlhüter, J.-M. George, M. Weigand, J. Raabe, V. Cros, and A. Fert, "Additive interfacial chiral interaction in multilayers for stabilization of small individual skyrmions at room temperature", Nature Nanotechnology 11, 444-448 (2016).

${ }^{60}$ G. Chen, A. Mascaraque, A. T. N'Diaye, and A. K. Schmid, "Room temperature skyrmion ground state stabilized through interlayer exchange coupling", Applied Physics Letters 106, 242404 (2015).

${ }^{61}$ N. S. Kiselev, a. N. Bogdanov, R. Schäfer, and U. K. Rößler, "Chiral skyrmions in thin magnetic films: new objects for magnetic storage technologies?", Journal of Physics D: Applied Physics 44, 392001 (2011). 
${ }^{62}$ S. Loth, S. Baumann, C. P. Lutz, D. M. Eigler, A. J. Heinrich, T. O. Wehling, S. Lounis, A. Lichtenstein, D. Pfannkuche, J. Wiebe, R. Wiesendanger, M. Garst, and A. Rosch, "Bistability in Atomic-Scale Antiferromagnets", Science 335, 196-199 (2012).

${ }^{63}$ F. Jonietz, S. Muhlbauer, C. Pfleiderer, A. Neubauer, W. Munzer, A. Bauer, T. Adams, R. Georgii, P. Boni, R. A. Duine, K. Everschor, M. Garst, and A. Rosch, "Spin Transfer Torques in MnSi at Ultralow Current Densities", Science 330, 1648-1651 (2010).

${ }^{64}$ A. Fert, "Magnetic and Transport Properties of Metallic Multilayers", Materials Science Forum 59-60, 439-480 (1991).

${ }^{65}$ R. A. Duine, K.-J. Lee, S. S. P. Parkin, and M. D. Stiles, "Synthetic Antiferromagnetic Spintronics: Part of a collection of reviews on antiferromagnetic spintronics", (2017).

${ }^{66}$ M. B. Salamon, S. Sinha, J. J. Rhyne, J. E. Cunningham, R. W. Erwin, J. Borchers, and C. P. Flynn, "Long-range incommensurate magnetic order in a Dy-Y multilayer", Physical Review Letters 56, 259-262 (1986).

${ }^{67}$ C. F. Majkrzak, J. W. Cable, J. Kwo, M. Hong, D. B. McWhan, Y. Yafet, J. V. Waszczak, and C. Vettier, "Observation of a Magnetic Antiphase Domain Structure with Long-Range Order in a Synthetic Gd-Y Superlattice", Physical Review Letters 56, 2700-2703 (1986).

${ }^{68}$ P. Grünberg, R. Schreiber, Y. Pang, M. B. Brodsky, and H. Sowers, "Layered Magnetic Structures: Evidence for Antiferromagnetic Coupling of Fe Layers across Cr Interlayers", Physical Review Letters 57, 2442-2445 (1986).

${ }^{69}$ S. S. P. Parkin, "Systematic variation of the strength and oscillation period of indirect magnetic exchange coupling through the $3 \mathrm{~d}, 4 \mathrm{~d}$, and 5d transition metals", Physical Review Letters 67, 3598-3601 (1991). 
${ }^{70}$ X. Zhang, Y. Zhou, and M. Ezawa, "ARTICLE Magnetic bilayerskyrmions without skyrmion Hall effect", Nature Communications 7, 10.1038/ncomms10293 (2016).

${ }^{71}$ A. K. Nandy, N. S. Kiselev, and S. Blügel, "Interlayer Exchange Coupling: A General Scheme Turning Chiral Magnets into Magnetic Multilayers Carrying Atomic-Scale Skyrmions", Physical Review Letters 116, 177202 (2016).

${ }^{72}$ T. Nakajima, H. Oike, A. Kikkawa, E. P. Gilbert, N. Booth, K. Kakurai, Y. Taguchi, Y. Tokura, F. Kagawa, and T.-h. Arima, "Skyrmion lattice structural transition in MnSi", Science Advances 3, e1602562 (2017).

${ }^{73}$ S.-Z. Lin and C. D. Batista, "Face Centered Cubic and Hexagonal Close Packed Skyrmion Crystals in Centrosymmetric Magnets", Physical Review Letters 120, 077202 (2018).

${ }^{74}$ S.-Z. Lin, A. Saxena, and C. D. Batista, "Skyrmion fractionalization and merons in chiral magnets with easy-plane anisotropy", Physical Review B 91, 224407 (2015).

${ }^{75}$ K. Karube, J. S. White, N. Reynolds, J. L. Gavilano, H. Oike, A. Kikkawa, F. Kagawa, Y. Tokunaga, H. M. Rønnow, Y. Tokura, and Y. Taguchi, "Robust metastable skyrmions and their triangular square lattice structural transition in a high-temperature chiral magnet", Nature Materials 15, 1237-1242 (2016).

${ }^{76}$ E. J. Mueller and T.-L. Ho, "Two-component Bose-Einstein condensates with a large number of vortices.", Physical review letters 88, 180403 (2002).

${ }^{77}$ A. Hrabec, N. A. Porter, A. Wells, M. J. Benitez, G. Burnell, S. McVitie, D. McGrouther, T. A. Moore, and C. H. Marrows, "Measuring and tailoring the Dzyaloshinskii-Moriya interaction in perpendicularly magnetized thin films", Physical Review B 90, 020402 (2014). 
${ }^{78}$ K. von Bergmann, A. Kubetzka, O. Pietzsch, and R. Wiesendanger, "Interface-induced chiral domain walls, spin spirals and skyrmions revealed by spin-polarized scanning tunneling microscopy", Journal of Physics: Condensed Matter 26, 394002 (2014).

${ }^{79}$ X. Yu, J. P. DeGrave, Y. Hara, T. Hara, S. Jin, and Y. Tokura, "Observation of the Magnetic Skyrmion Lattice in a MnSi Nanowire by Lorentz TEM", Nano Letters 13, 3755-3759 (2013).

${ }^{80}$ E. van Walsem, R. A. Duine, and M. H. D. Guimarães, "Layer effects on the magnetic textures in magnets with local inversion asymmetry", Phys. Rev. B 102, 174403 (2020).

${ }^{81}$ M. Bode, M. Heide, K. von Bergmann, P. Ferriani, S. Heinze, G. Bihlmayer, A. Kubetzka, O. Pietzsch, S. Blügel, and R. Wiesendanger, "Chiral magnetic order at surfaces driven by inversion asymmetry", Nature 447, 190-193 (2007).

${ }^{82} \mathrm{M}$. Heide, G. Bihlmayer, and S. Blügel, "Dzyaloshinskii-Moriya interaction accounting for the orientation of magnetic domains in ultrathin films: Fe/W(110)", Physical Review B 78, 140403 (2008).

${ }^{83}$ A. Thiaville, S. Rohart, É. Jué, V. Cros, and A. Fert, "Dynamics of Dzyaloshinskii domain walls in ultrathin magnetic films", EPL (Europhysics Letters) 100, 57002 (2012).

${ }^{84}$ A. K. Geim and I. V. Grigorieva, "Van der Waals heterostructures", Nature 499, 419-425 (2013).

${ }^{85}$ M. Gibertini, M. Koperski, A. F. Morpurgo, and K. S. Novoselov, Magnetic 2D materials and heterostructures, 2019.

${ }^{86}$ K. S. Novoselov, A. K. Geim, S. V. Morozov, D. Jiang, Y. Zhang, S. V. Dubonos, I. V. Grigorieva, A. A. Firsov, K. S. Novoselov, A. K. Geim, S. V. Morozov, D. Jiang, Y. Zhang, S. V. Dubonos, 
I. V. Grigorieva, and A. A. Firsov, "Electric Field Effect in Atomically Thin Carbon Films", Sci 306, 666-669 (2004).

${ }^{87}$ B. Huang, G. Clark, E. Navarro-Moratalla, D. R. Klein, R. Cheng, K. L. Seyler, D. Zhong, E. Schmidgall, M. A. McGuire, D. H. Cobden, W. Yao, D. Xiao, P. Jarillo-Herrero, and X. Xu, "Layer-dependent ferromagnetism in a van der Waals crystal down to the monolayer limit", Nature 546, 270-273 (2017).

${ }^{88}$ X. Zhang, Q. Liu, J. W. Luo, A. J. Freeman, and A. Zunger, "Hidden spin polarization in inversion-symmetric bulk crystals", Nature Physics 10, 387-393 (2014).

${ }^{89}$ J. M. Riley, F. Mazzola, M. Dendzik, M. Michiardi, T. Takayama, L. Bawden, C. Granerød, M. Leandersson, T. Balasubramanian, M. Hoesch, T. K. Kim, H. Takagi, W. Meevasana, P. Hofmann, M. S. Bahramy, J. W. Wells, and P. D. King, "Direct observation of spin-polarized bulk bands in an inversion-symmetric semiconductor", Nature Physics 10, 835-839 (2014).

${ }^{90}$ R. Bertoni, C. W. Nicholson, L. Waldecker, H. Hübener, C. Monney, U. De Giovannini, M. Puppin, M. Hoesch, E. Springate, R. T. Chapman, C. Cacho, M. Wolf, A. Rubio, and R. Ernstorfer, "Generation and Evolution of Spin-, Valley-, and LayerPolarized Excited Carriers in Inversion-Symmetric WSe2", Physical Review Letters 117, 277201 (2016).

${ }^{91}$ E. Razzoli, T. Jaouen, M. L. Mottas, B. Hildebrand, G. Monney, A. Pisoni, S. Muff, M. Fanciulli, N. C. Plumb, V. A. Rogalev, V. N. Strocov, J. Mesot, M. Shi, J. H. Dil, H. Beck, and P. Aebi, "Selective Probing of Hidden Spin-Polarized States in InversionSymmetric Bulk MoS2", Physical Review Letters 118, 086402 (2017).

${ }^{92}$ M. H. D. Guimarães and B. Koopmans, "Spin Accumulation and Dynamics in Inversion-Symmetric van der Waals Crystals", Physical Review Letters 120, 266801 (2018). 
${ }^{93}$ K.-W. Kim, K.-W. Moon, and K. Everschor-Sitte, "Asymmetric Skyrmion Hall Effect in Systems with Hybrid DzyaloshinskiiMoriya Interaction", (2018).

${ }^{94} \varnothing$. Johansen, V. Risinggård, A. Sudbø, J. Linder, and A. Brataas, "Current Control of Magnetism in Two-Dimensional Fe3GeTe2", Physical Review Letters 122, 217203 (2019).

${ }^{95}$ J. Lucassen, M. J. Meijer, M. C. H. de Jong, R. A. Duine, H. J. M. Swagten, B. Koopmans, and R. Lavrijsen, "Stabilizing chiral spin-structures via an alternating Dzyaloshinskii-Moriya interaction", (2020).

${ }^{96}$ T. Li, S. Jiang, N. Sivadas, Z. Wang, Y. Xu, D. Weber, J. E. Goldberger, K. Watanabe, T. Taniguchi, C. J. Fennie, K. Fai Mak, and J. Shan, Pressure-controlled interlayer magnetism in atomically thin CrI3, 2019.

${ }^{97}$ X. Zhang, M. Ezawa, and Y. Zhou, "Thermally stable magnetic skyrmions in multilayer synthetic antiferromagnetic racetracks", Physical Review B 94, 604406 (2016).

${ }^{98}$ A. Neubauer, C. Pfleiderer, B. Binz, A. Rosch, R. Ritz, P. G. Niklowitz, and P. Böni, "Topological Hall Effect in the A Phase of MnSi", Physical Review Letters 102, 186602 (2009).

${ }^{99}$ K. Litzius, I. Lemesh, B. Krüger, P. Bassirian, L. Caretta, K. Richter, F. Büttner, K. Sato, O. A. Tretiakov, J. Förster, R. M. Reeve, M. Weigand, I. Bykova, H. Stoll, G. Schütz, G. S. Beach, and M. Klaüi, "Skyrmion Hall effect revealed by direct timeresolved X-ray microscopy", Nature Physics 13, 170-175 (2017). ${ }^{100}$ P. Milde, D. Köhler, J. Seidel, L. M. Eng, A. Bauer, A. Chacon, J. Kindervater, S. Mühlbauer, C. Pfleiderer, S. Buhrandt, C. Schütte, and A. Rosch, "Unwinding of a skyrmion lattice by magnetic monopoles", Science 340, 1076-1080 (2013). 
${ }^{101}$ W. Jiang, X. Zhang, G. Yu, W. Zhang, X. Wang, M. Benjamin Jungfleisch, J. E. Pearson, X. Cheng, O. Heinonen, K. L. Wang, Y. Zhou, A. Hoffmann, and S. G. Te Velthuis, "Direct observation of the skyrmion Hall effect", Nature Physics 13, 162-169 (2017).

${ }^{102}$ A. O. Leonov, Y. Togawa, T. L. Monchesky, A. N. Bogdanov, J. Kishine, Y. Kousaka, M. Miyagawa, T. Koyama, J. Akimitsu, T. Koyama, K. Harada, S. Mori, D. McGrouther, R. Lamb, M. Krajnak, S. McVitie, R. L. Stamps, and K. Inoue, "Chiral Surface Twists and Skyrmion Stability in Nanolayers of Cubic Helimagnets", Physical Review Letters 117, 087202 (2016).

${ }^{103}$ M. Nagao, Y. G. So, H. Yoshida, M. Isobe, T. Hara, K. Ishizuka, and K. Kimoto, "Direct observation and dynamics of spontaneous skyrmion-like magnetic domains in a ferromagnet", Nature Nanotechnology 8, 10.1038/nnano.2013.69 (2013).

${ }^{104}$ S. Woo, K. M. Song, X. Zhang, Y. Zhou, M. Ezawa, X. Liu, S. Finizio, J. Raabe, N. J. Lee, S. I. Kim, S. Y. Park, Y. Kim, J. Y. Kim, D. Lee, O. Lee, J. W. Choi, B. C. Min, H. C. Koo, and J. Chang, "Current-driven dynamics and inhibition of the skyrmion Hall effect of ferrimagnetic skyrmions in GdFeCo films", Nature Communications 9, 10.1038/s41467-018-03378-7 (2018).

${ }^{105}$ N. E. Penthorn, X. Hao, Z. Wang, Y. Huai, and H. W. Jiang, "Experimental Observation of Single Skyrmion Signatures in a Magnetic Tunnel Junction", Physical Review Letters 122, 257201 (2019).

${ }^{106}$ C. Zhang, J. Wang, C. Jin, Z. Zeng, H. Xia, J. Wang, and Q. Liu, "Spin current pumped by confined breathing skyrmion", New Journal of Physics 22, 53029 (2020).

${ }^{107}$ M. Garst, J. Waizner, and D. Grundler, Collective spin excitations of helices and magnetic skyrmions: Review and perspectives of magnonics in non-centrosymmetric magnets, 2017. 
${ }^{108}$ T. Schwarze, J. Waizner, M. Garst, A. Bauer, I. Stasinopoulos, H. Berger, C. Pfleiderer, and D. Grundler, "Universal helimagnon and skyrmion excitations in metallic, semiconducting and insulating chiral magnets", Nature Materials 14, 478-483 (2015).

${ }^{109}$ Y. Onose, Y. Okamura, S. Seki, S. Ishiwata, and Y. Tokura, "Observation of magnetic excitations of skyrmion crystal in a helimagnetic insulator $\mathrm{Cu} 2 \mathrm{OSeO} 3$ ", Physical Review Letters 109, 037603 (2012).

${ }^{110}$ C. Psaroudaki, S. Hoffman, J. Klinovaja, and D. Loss, "Quantum dynamics of skyrmions in chiral magnets", Physical Review X 7, 10.1103/PhysRevX.7.041045 (2017).

${ }^{111}$ A. N. Bogdanov and U. B. Rößler, "Chiral symmetry breaking in magnetic thin films and multilayers", Physical Review Letters 87, 37203-1-37203-4 (2001).

${ }^{112}$ A. Roldán-Molina, M. J. Santander, A. S. Núñez, and J. Fernández-Rossier, "Quantum theory of spin waves in finite chiral spin chains", Physical Review B - Condensed Matter and Materials Physics 89, 10.1103/PhysRevB.89.054403 (2014). 
A Addendum

Nederlandse samenvatting

Dankwoord

About the author
103

109

111 



\section{Nederlandse samenvatting}

Het is verbazingwekkend hoeveel magneten je gebruikt in je dagelijks leven. Niet alleen op je koelkast zitten magneten, maar ook in de speaker van je mobieltje en in de harde schijf van je computer wordt er gebruik van gemaakt. In de wedloop van technologiebedrijven worden er steeds snellere harde schijven met meer opslag ontwikkeld. Echter, de huidige technieken lopen tegen een limiet aan. Daarom zijn er nieuwe technieken nodig om harde schijven mee te bouwen. Een van de nieuwe technieken waar veel interesse in is zijn hele dunne magneten waar bijzondere magnetische structuren in voorkomen. In dit proefschrift hebben we onderzoek gedaan naar dit soort magneten. In deze samenvatting willen we uitleggen hoe een magneet werkt, wat voor bijzondere structuren er in magneten bestaan en waarom deze zo nuttig zijn voor toepassingen als harde schijven. Daarna zullen we kort uitleggen wat we in het bijzonder hebben onderzocht aan deze magneten.

\section{Hoe werkt een magneet?}

Een permanente magneet is een stuk materiaal dat van zichzelf magnetisme vertoond: er vormt zich een magneetveld om het materiaal met twee polen die altijd dezelfde kant op staan. Maar waarom blijft het ene stuk materiaal wel aan een koelkast hangen en de andere niet? Magneten bestaan uit allemaal atomen die op zichzelf ook weer kleine magneetjes zijn. Dit kleine magneetje komt 
van een kwantummechanisch verschijnsel dat spin heet en wordt vaak met een pijl aangegeven in tekeningen. In een magneet zijn alle spins dezelfde kant op gericht. Het magnetisch moment van alle atomen werken dan samen en het totale magneetveld van het materiaal staat dan in dezelfde richting. materiaal met deze eigenschap noemen we dan een ferromagneet, in Fig. A.1(a) hebben we een voorbeeld van een ferromagneet getekend waar je kan zien dat alle spins dezelfde kant op zijn gericht. De atomen weten welke kant ze op moeten staan omdat ze wisselwerken met hun buren. In ferromagneten is die wisselwerking dus erg goed. Maar er bestaan ook materialen waar deze wisselwerking niet sterk genoeg is of juist tegenwerkt. In een antiferromagneet willen alle spins om en om staan. In dit geval heffen de kleine magneetvelden elkaar op en is het materiaal juist niet magnetisch. Een voorbeeld hiervan hebben we getekend in Fig. A.1 (b).

(a)

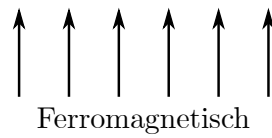

(b)

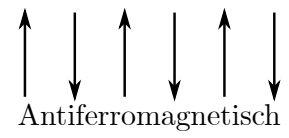

(c)

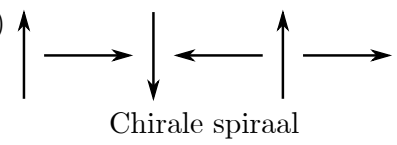

Figuur A.1: Schematische tekening van verscheidene spin structuren.

$$
\text { materiaal }
$$

\section{Chirale structuren}

De wisselwerking tussen de spins gaat niet alleen maar of de spin parallel of anti-parallel staat. Bijvoorbeeld als je een dunne laag platinum onder een magneet plakt wordt de wisselwerking verstoord. In dit geval willen twee atomen die naast elkaar staan in de magneet juist niet parallel aan elkaar staan, maar loodrecht zoals in Fig. A.1 (c). Omdat de laag platinum maar aan een kant van je magneet zit is deze interactie altijd in dezelfde richting, en dus 
chiraal. Als je zulke wisselwerking in een dunne magneet hebt krijg je een spiraal structuur zoals op de achterkant van dit proefschrift is getekend. Dit is een oppervlakte effect, namelijk het wordt veroorzaakt door het raakvlak tussen de magneet en het platinum en is dus het sterkst dicht bij de oppervlakte. Een zo dun mogelijke magneet heeft hier het meeste effect van en daarom bestuderen we dunne tweedimensionale magneten in dit proefschrift.

Als je een magnetisch veld aanlegt over een ferromagneet, bijvoorbeeld door hem midden in een grote elektrische spoel te stoppen, dan willen de spins juist in de richting van dit magneetveld staan. In het geval van een magneet met spiralen die we net hierboven besproken hebben ontstaat er een competitie tussen de spiralen en het magneetveld. Hierdoor vormen er bijzondere structuren die skyrmionen genoemd worden. In een skyrmion vormen alle spins een soort kruin zoals we in Fig. A.2 (a) getekend hebben. Deze hele verzameling van spins die dat kruin vormen is de skyrmion.

Skyrmionen zijn erg interessant omdat ze heel robuust zijn, ze behouden altijd hun vorm. Daarom kunnen we de skyrmionen door het systeem bewegen zonder dat ze kapot gaan. Daarnaast kunnen ze ook nog erg klein zijn, tot enkele nanometers in diameter. Dit maakt ze erg geschikt voor harde schijf toepassingen: er passen veel skyrmions op een chip, en je raakt ze niet kwijt. Een skyrmion is zo stevig vanwege een wiskundig "slot" uit de topologie. Als je van een skyrmion bijhoudt in welke richting de spins allemaal op wijzen dan kan je ze precies op een bol afbeelden. In Fig. A.2 (b) hebben we dat getekend. De chiraliteit die we eerder besproken hebben heeft hier een grote rol in.

\section{Dit proefschrift}

In Chapter 2 hebben we twee dunne magnetische lagen gesimuleerd waar skyrmionen in voor komen. In dit systeem kwamen zo veel skyrmionen voor dat ze een rooster vormen. Omdat skyrmi- 
(a)

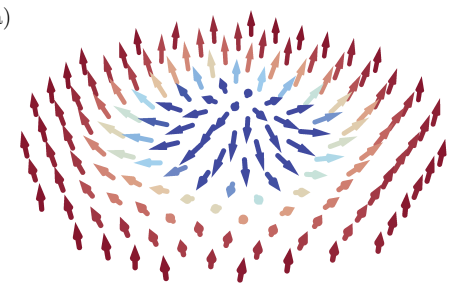

(b)

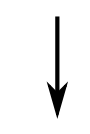

b)

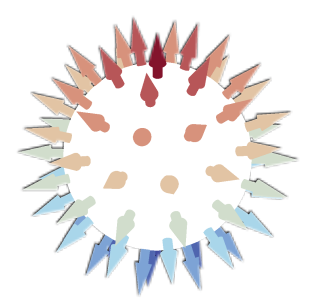

Figuur A.2: Schematische tekening van (a) een skyrmion en (b) een bol waar alle spins van een skyrmion op afgebeeld zijn. 
onen elkaar af stoten nemen ze plaatst op een driehoekig rooster. Vervolgens hebben we deze als twee boterhammen boven op elkaar gelegd. De combinatie van die twee lagen magnetisch materiaal heet een synthetische antiferromagneet omdat de koppeling tussen de lagen zo wel aantrekkend als afstotend kan werken afhankelijk van de afstand. In Fig. A.3 (a) heb we geschetst hoe twee driehoekige skyrmion roosters (groen en rood) plaatsen als ze elkaar afstoten. Maar wat bleek is dat als we de koppeling tussen die twee lagen sterker maken, dan veranderd het rooster in een vierkant rooster, zoals Fig. A.3 (b). Omdat de koppeling tussen twee lagen aan te passen is met externe krachten zoals een elektrisch veld of gewoon door er op te drukken is het mogelijk om te schakelen tussen verschillende roosterconfiguraties van skyrmionen.

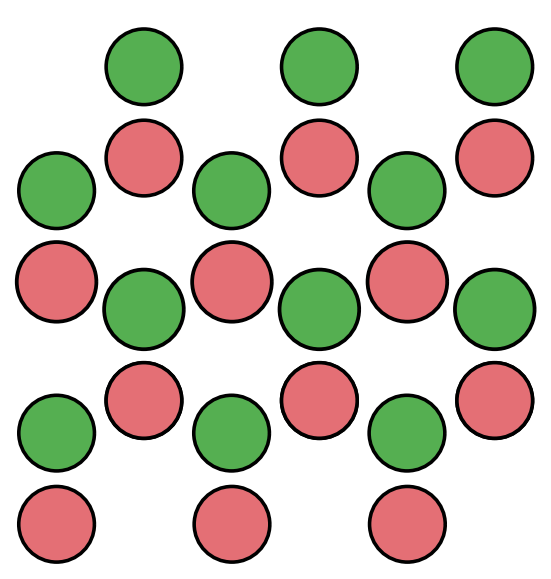

(a)

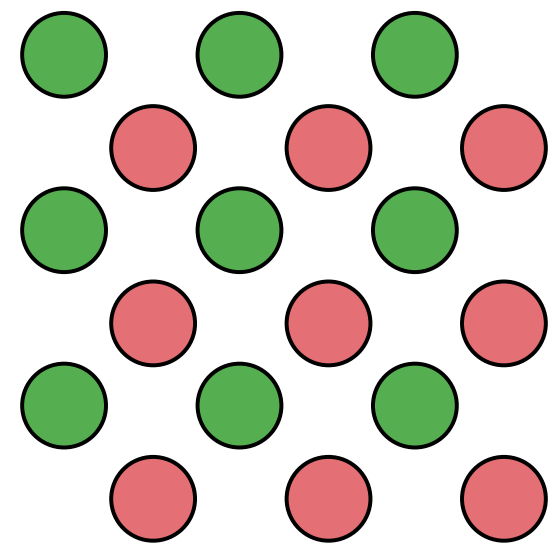

(b)

Figuur A.3: Schematische tekening van twee skyrmion roosters boven elkaar. Rooster 1 is met groene cirkels afgebeeld en rooster 2 met rode cirkels. In (a) zijn twee driehoekige rooters getekend en in (b) twee vierkante. 
In Chapter 3 hebben we chirale spiralen bestudeerd. Daar hebben we meerdere magnetische lagen met spiralen op elkaar gestapeld zodat dat draairichting van de spiralen in elke laag andersom is. We hebben gekeken hoe de spiralen hier op reageren. Het blijkt als je de koppeling tussen deze lagen heel sterk maakt de golflengte van de spiralen veranderd. Een belangrijk gegeven is hoeveel lagen het systeem heeft. Bij een oneven aantal lagen wordt de golflengte veel langer, evenredig met het aantal lagen. En bij een even aantal lagen verdwijnt de spiraal zelfs. Dit is allemaal, net zoals onze vorige bevinding, extern te beïnvloeden waardoor dat mogelijkheden geeft voor toepassingen.

In Chapter 4 zijn we teruggegaan naar de skyrmionen. Hier hebben we twee losse skyrmionen boven elkaar geplaatst. Daar hebben we uitgerekend hoe we zulke skyrmionen kunnen detecteren als we het magneetveld niet kunnen zien. Dit kunnen we doen door microgolven (zoals in de magnetron) door het systeem te sturen. Skyrmionen resoneren op een bepaalde manier met deze microgolven. We weten nu ook welke karakteristieken we kunnen verwachten als er twee skyrmionen boven elkaar zitten. Hiervoor was het alleen bekend hoe een enkele laag skyrmionen reageert. 


\section{Dankwoord}

Allereerst wil ik Rembert bedanken dat je mij de kans te geven een promotie traject bij jou te doen. Ik ben zeer dankbaar voor je geduldige begeleiding en de vele leuke gesprekken over het fietsen. Verder wil ik Patrick en Joren bedanken voor de medewerking tijdens het Theory for Technology project en het delen van de data voor het bijbehorende hoofdstuk. Thank you Marcos for your discussions and contributions to our paper. Reinoud en Juriaan, bedankt voor jullie wetenschappelijke bijdrages en het toffe symposium in Parijs. Rick bedankt dat je me op gang hebt geholpen.

Thanks to everybody in Rembert's group for the weekly lunch meetings and the fun scientific talks. Daarnaast wil ik iedereen bedanken waar ik de afgelopen jaren fijne uurtjes mee heb doorgebracht op het ITF. In het bijzonder Sander, Sander, Tycho, Benedict, Erik, Gerwin, Guido, Camilo, Nick, Juriaan, Sonja, Rafaelle, Jianzen, Watse, Mathijs en Bram. Bedankt voor alle gezelligheid en de goede discussies aan de lunchtafel. Thanks to all my office mates, Sander, Mikael, Tom, Pjotr en Anja, over the years to make the office a more comfortable place day by day.

Ik wil iedereen bedanken die mij vergezeld hebben in de nodige ontspanning naast mijn $\mathrm{PhD}$, de schaduwhark voor de fantastische vakanties, boeiende pizzaboekenclubs en geweldige gesprekken. Iedereen op scouting bedankt voor de wekelijkse uitdagingen. Martijn en Thomas bedankt voor de jarenlang vriendschap. En dan Sarah nog in het speciaal voor het ontwerpen van mijn cover, het 
aan horen van mijn gezeur en het delen van leuke linkjes.

Ik wil mijn Emiel, Monica en Koen bedanken voor de afgelopen 5 jaar zeer fijn samenwonen en dat we elkaar door de corona crisis aan het helpen zijn en tegelijk ook nog fulltime kantoorgenootjes zijn geworden. En dan wil ik nog speciaal Wendy bedanken voor alle steun tijdens de laatste eindsprintjes van mijn $\mathrm{PhD}$ en het experimentele bewijs van Michael Boogerds wijsheid dat de laatste loodjes op de fiets altijd het zwaarst zijn.

Ten slotte wil ik mijn ouders en Soizic bedanken dat jullie mij leerden ergens voor te gaan en vervolgens om daarmee door te zetten, en dat jullie mij daarin altijd overal in gesteund hebben. 


\section{About the author}

Etienne van Walsem was born on the 31st of July 1991 in Maarssen. After completing his vwo in 2009 at the Gerrit Rietveld College in Utrecht he went on to obtain his Bachelor of Physics and Astronomy at the University of Amsterdam. He spent part of his Bachelor at the National University of Singapore during an exchange semester. Subsequently, he finished his Master of Physics at the University of Amsterdam in 2015 with a master thesis on Quantum Monte Carlo simulations of the Bose-Hubbard model on a Kagome Lattice. In the same year he obtained a $\mathrm{PhD}$ position at Utrecht University under the supervision of Rembert Duine. This thesis is an account of the research conducted during this program. In his spare time Etienne enjoys running, cycling and organizing weekly meetings at scouting Marsua. 